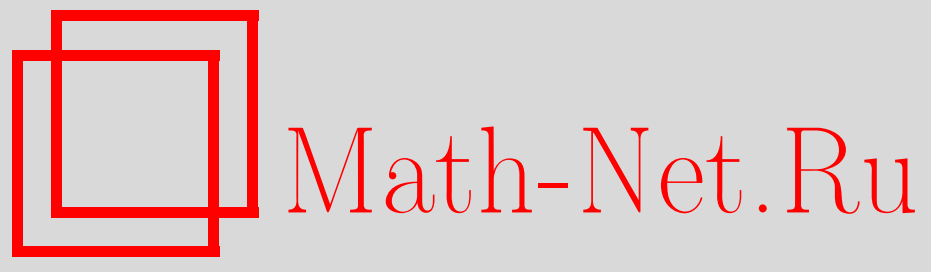

И. А. Тайманов, Двумерный оператор Дирака и теория поверхностей, УМН, 2006, том 61, выпуск 1, 85-164

DOI: https://doi.org/10.4213/rm1716

Использование Общероссийского математического портала Math-Net.Ru подразумевает, что вы прочитали и согласны с пользовательским соглашением http://www . mathnet.ru/rus/agreement

Параметры загрузки:

IP: 3.82 .47 .9

26 апреля 2023 г., 04:14:08

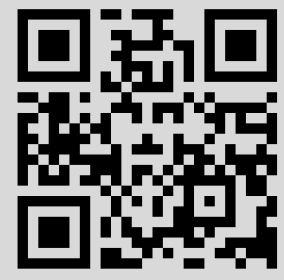




\section{Двумерный оператор Дирака и теория поверхностей}

\section{И. А. Тайманов}

В работе дан обзор по представлениям Вейерштрасса поверхностей в трех- и четырехмерных пространствах, их приложениям к теории функционала Уиллмора и по связанным с ними проблемам спектральной теории двумерного оператора Дирака с периодическими коэффициентами.

Библиография: 138 названий.

\section{СОДЕРЖАНИЕ}

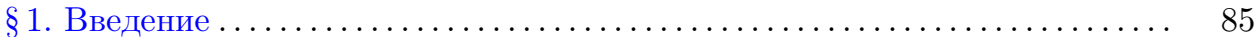

$\S 2$. Представления поверхностей в трех- и четырехмерных пространствах 87

$\S 3$. Интегрируемые деформации поверхностей ................... 104

$\S 4$. Спектральные кривые $\ldots \ldots \ldots \ldots \ldots \ldots \ldots \ldots \ldots \ldots \ldots \ldots \ldots \ldots \ldots \ldots \ldots . \ldots \ldots$

$\S 5$. Функционал Уиллмора .................................. 129

Приложение 1. О существовании спектральной кривой оператора Дирака

с $L_{2}$-потенциалами ............................... 144

Приложение 2. Конформное отображение Гаусса и конформная площадь 151

Приложение 3. Обратная задача рассеяния для оператора Дирака на пря-

мой и формулы следов .............................. 154

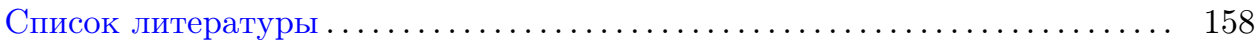

\section{§ 1. Введение}

В этой работе мы даем обзор некоторых результатов и проблем, относящихся к глобальным представлениям поверхностей в трех- и четырехмерных пространствах в терминах решений уравнения Дирака и применению этих конструкций к изучению функционала Уиллмора и его обобщений.

Эта деятельность началась десять лет назад [1]. При этом подходе отображение Гаусса поверхности представляется в терминах решения $\psi$ уравнения Дирака

$$
\mathscr{D} \psi=0,
$$

где $\mathscr{D}$ - оператор Дирака с потенциалами

$$
\mathscr{D}=\left(\begin{array}{cc}
0 & \partial \\
-\bar{\partial} & 0
\end{array}\right)+\left(\begin{array}{cc}
U & 0 \\
0 & V
\end{array}\right) .
$$

Работа выполнена при поддержке Российского фонда фундаментальных исследований.

(C) И.А. ТАЙманов, 2006 
Такое представление для поверхностей в $\mathbb{R}^{3}$ имеет различные формы, но в этом виде, связанном с уравнением Дирака, оно впервые было выписано для построения поверхностей, допускающих солитонные деформации, в [2], где эти деформации были также введены.

Появление оператора с хорошо развитой спектральной теорией сделало возможным использовать эту теорию для изучения задач глобальной теории поверхностей. Более того, этот подход объясняет важность функционала Уиллмора, поскольку для поверхностей в $\mathbb{R}^{3}$ он с точностью до множителя является квадратом $L_{2}$-нормы потенциала $U=V=\bar{U}$ оператора $\mathscr{D}$ [1].

Подход к доказательству гипотезы Уиллмора, предложенный в [1], [3] и основанный на теории спектральных кривых (на одном уровне энергии) [4], привел к очень интересной работе Шмидта [5], где был достигнут существенный прогресс, но сама гипотеза осталась недоказанной.

При этом спектральная кривая оператора $\mathscr{D}$ с двояко-периодическими потенциалами приводит к понятию спектральной кривой тора в $\mathbb{R}^{3}[3]$, в которой закодирован большой объем информации о геометрии поверхности.

Другой подход к получению нижних оценок для функционала Уиллмора привлек методы обратной задачи рассеяния и алгебраической геометрии кривых и привел к получению оценок, квадратичных по размерности ядра оператора $\mathscr{D}$. Они впервые были получены для сфер вращения и некоторых их обобщений и высказаны в качестве гипотезы для всех сфер в [6] с помощью обратной задачи рассеяния и доказаны во всей полноте для поверхностей любого рода в [7], где теория алгебраических кривых была удивительным и необычным способом применена к теории поверхностей.

Позднее это представление было обобщено для поверхностей в $\mathbb{R}^{4}[8],[9]$ и трехмерных группах Ли [10], [11]. В [12], [7] было предложено с самого начала рассматривать представления поверхностей в $\mathbb{R}^{3}$ и $\mathbb{R}^{4}$ в рамках конформной геометрии. Но для некоммутативных некомпактных групп аналоги функционала Уиллмора принимают вид

$$
\int\left(\alpha H^{2}+\beta \widehat{K}+\gamma\right) d \mu
$$

где $H$ - средняя кривизна, $\widehat{K}$ - секционная кривизна объемлющего пространства вдоль касательной плоскости к поверхности и $d \mu-$ индуцированная мера на поверхности. Отметим, что функционалы похожего вида

$$
\int\left(\alpha H^{2}+\beta K+\gamma\right) d \mu
$$

широко известны в физике как функционалы Хелфриха [13] (см. также, например, [14], [15]) и для значений $\alpha, \beta, \gamma$ в общем положении они не конформноинвариантны даже для поверхностей в $\mathbb{R}^{3}$. Здесь член, содержащий гауссову кривизну $K$, не сводится к топологическому члену для поверхностей с границей, которые интересны для физических приложений.

Хотя до настоящего времени эти представления применялись в основном $\mathrm{K}$ задачам, связанным с функционалом Уиллмора и его обобщениями, мы уверены, что они могут эффективно использоваться для изучения других проблем глобальной теории поверхностей. 


\section{§ 2. Представления поверхностей в трех- и четырехмерных пространствах}

2.1. Обобщенные формулы Вейерштрасса для поверхностей в $\mathbb{R}^{3}$. Многообразия Грассмана (грассманианы) ориентированных двумерных плоскостей в $\mathbb{R}^{n}$ диффеоморфны квадрикам в $\mathbb{C} P^{n-1}$.

Действительно, возьмем двумерную плоскость и выберем для нее положительно ориентированный ортонормированный базис $u=\left(u_{1}, \ldots, u_{n}\right)$, $v=\left(v_{1}, \ldots, v_{n}\right)$, т.е. $|u|=|v|, \quad(u, v)=0$. Он задается таким вектором $y=u+i v \in \mathbb{C}^{n}$, что

$$
y_{1}^{2}+\cdots+y_{n}^{2}=[(u, u)-(v, v)]+2 i(u, v)=0 .
$$

Плоскость определяет такой базис с точностью до вращений плоскости на угол $\varphi, 0 \leqslant \varphi \leqslant 2 \pi$, приводящих к преобразованиям $y \rightarrow r e^{i \varphi} y$. Следовательно, грассманиан $\widetilde{G}_{n, 2}$ ориентированных двумерный плоскостей в $\mathbb{R}^{n}$ диффеоморфен квадрике

$$
y_{1}^{2}+\cdots+y_{n}^{2}=0, \quad\left(y_{1}: \ldots: y_{n}\right) \in \mathbb{C} P^{n-1},
$$

где $\left(y_{1}: \ldots: y_{n}\right)$ - однородные координаты в $\mathbb{C} P^{n-1}$. Грассманиан $G_{n, 2}$ неориентированных двумерных плоскостей в $\mathbb{R}^{n}$ является фактор-пространством многообразия $\widetilde{G}_{n, 2}$ по свободному действию антиголоморфной инволюции $y \rightarrow \bar{y}$.

Для погруженной поверхности

$$
f: \Sigma \rightarrow \mathbb{R}^{n}
$$

с (локальным) конформным параметром $z$, отображение Гаусса имеет вид

$$
\Sigma \rightarrow \widetilde{G}_{n, 2}: P \rightarrow\left(x_{z}^{1}(P): \ldots: x_{z}^{n}(P)\right),
$$

где $x^{1}, \ldots, x^{n}$ - евклидовы координаты в $\mathbb{R}^{n}$ и $P \in \Sigma$.

Существуют только два случая, когда грассманиан допускает рациональную параметризацию:

$$
\widetilde{G}_{3,2}=\mathbb{C} P^{1}, \quad \widetilde{G}_{4,2}=\mathbb{C} P^{1} \times \mathbb{C} P^{1},
$$

и только в этих двух случаях существуют представления Вейерштрасса поверхностей.

Рассмотрим сначала поверхности в $\mathbb{R}^{3}$.

Грассманиан $\widetilde{G}_{3,2}$ является квадрикой

$$
y_{1}^{2}+y_{2}^{2}+y_{3}^{2}=0
$$

имеющей следующую рациональную параметризацию ${ }^{1}$ :

$$
y_{1}=\frac{i}{2}\left(b^{2}+a^{2}\right), \quad y_{2}=\frac{1}{2}\left(b^{2}-a^{2}\right), \quad y_{3}=a b, \quad(a: b) \in \mathbb{C} P^{1} .
$$

Положим

$$
\psi_{1}=a, \quad \psi_{2}=\bar{b}
$$

\footnotetext{
${ }^{1}$ Она известна в теории чисел как представление Лагранжа всех целочисленных решений уравнения $x^{2}+y^{2}=z^{2}$.
} 
и подставим эти выражения в формулы для $x_{z}^{k}=y_{k}, k=1,2,3$. Так как $x^{k} \in \mathbb{R}$ для всех $k$, мы имеем

$$
\operatorname{Im} x_{z \bar{z}}^{k}=0, \quad k=1,2,3 .
$$

В терминах $\psi$ это условие принимает вид уравнения Дирака

$$
\mathscr{D} \psi=\left[\left(\begin{array}{cc}
0 & \partial \\
-\bar{\partial} & 0
\end{array}\right)+\left(\begin{array}{cc}
U & 0 \\
0 & U
\end{array}\right)\right]\left(\begin{array}{l}
\psi_{1} \\
\psi_{2}
\end{array}\right)=0, \quad U=\bar{U} .
$$

Более того, если для комплекснозначной функции $f$ мы имеем $\operatorname{Im} f_{\bar{z}}=0$, то локально $f=g_{z}$, где $g$ - вещественнозначная функция вида

$$
g=\int[\operatorname{Re} f d x-\operatorname{Im} f d y] .
$$

Мы имеем следующую теорему.

Теорема 1. 1) [2] Если $\psi$ удовлетворяет уравнению Дирака (1), то формульь

$$
x^{k}=x^{k}(0)+\int\left(x_{z}^{k} d z+\bar{x}_{z}^{k} d \bar{z}\right), \quad k=1,2,3,
$$

¿əe

$$
x_{z}^{1}=\frac{i}{2}\left(\bar{\psi}_{2}^{2}+\psi_{1}^{2}\right), \quad x_{z}^{2}=\frac{1}{2}\left(\bar{\psi}_{2}^{2}-\psi_{1}^{2}\right), \quad x_{z}^{3}=\psi_{1} \bar{\psi}_{2}
$$

задают поверхность в $\mathbb{R}^{3}$.

2) [1] Каждая гладкая поверхность в $\mathbb{R}^{3}$ локально задается формулами вида (2) $u(3)$.

Доказательство второго утверждения дано выше, а первое утверждение доказывается следующим образом: согласно уравнению Дирака, подынтегральные выражения в (2) являются замкнутыми формами и по теореме Стокса значения интегралов не зависят от выбора пути в односвязной области из $\mathbb{C}$.

Это представление поверхности называется представлением Вейеритрасса. При $U=0$ оно сводится к классическому представлению Вейерштрасса (или Вейерштрасса-Эннепера) минимальных поверхностей.

Следующее предложение выводится прямыми вычислениями.

ПреДЛОЖЕНИЕ 1. Для поверхности $\Sigma$, заданной формулами (2) и (3),

1) z- конформныц параметр на поверхности и индуцированная метрика имеет вид

$$
d s^{2}=e^{2 \alpha} d z d \bar{z}, \quad e^{\alpha}=\left|\psi_{1}\right|^{2}+\left|\psi_{2}\right|^{2},
$$

2) потенциал U оператора Дирака равен

$$
U=\frac{H e^{\alpha}}{2},
$$

где $H-$ средняя кривизна, ${ }^{2}$ т.е. $H=\frac{\varkappa_{1}+\varkappa_{2}}{2}$, где $\varkappa_{1}, \varkappa_{2}-$ главные кривизнь,

\footnotetext{
2Заметим, что вектор нормали $N$ удовлетворяет уравнению

$$
\Delta f=2 H N,
$$

где $\Delta=4 e^{-2 \alpha} \partial \bar{\partial}$ - оператор Лапласа-Бельтрами на поверхности.
} 
3) дифференциал Хопфа равен $A d z^{2}=\left(f_{z z}, N\right) d z^{2} u$

$$
|A|^{2}=\frac{\left(\varkappa_{1}-\varkappa_{2}\right)^{2} e^{4 \alpha}}{16}, \quad A=\bar{\psi}_{2} \partial \psi_{1}-\psi_{1} \partial \bar{\psi}_{2},
$$

4) уравнения Гаусса-Вейнгартена принимают вид

$$
\left[\frac{\partial}{\partial z}-\left(\begin{array}{cc}
\alpha_{z} & A e^{-\alpha} \\
-U & 0
\end{array}\right)\right] \psi=\left[\frac{\partial}{\partial \bar{z}}-\left(\begin{array}{cc}
0 & U \\
-\bar{A} e^{-\alpha} & \alpha_{\bar{z}}
\end{array}\right)\right] \psi=0,
$$

5) условия совместности для уравнений Гаусса-Вейнгартена - это уравнения Гаусса-Кодации вида

$$
A_{\bar{z}}=\left(U_{z}-\alpha_{z} U\right) e^{\alpha}, \quad \alpha_{z \bar{z}}+U^{2}-A \bar{A} e^{-2 \alpha}=0
$$

и гауссова кривизна равна $K=-4 e^{-2 \alpha} \alpha_{z \bar{z}}$.

Легко заметить, что если $\varphi$ удовлетворяет уравнению Дирака (1), то векторфункция $\varphi^{*}$, определенная формулой

$$
\varphi=\left(\begin{array}{l}
\varphi_{1} \\
\varphi_{2}
\end{array}\right) \rightarrow \varphi^{*}=\left(\begin{array}{c}
-\bar{\varphi}_{2} \\
\bar{\varphi}_{1}
\end{array}\right)
$$

также удовлетворяет уравнению Дирака.

Отождествим $\mathbb{R}^{3}$ с линейным пространством $(2 \times 2)$-матриц, порожденным над $\mathbb{R}$ матрицами

$$
e_{1}=\left(\begin{array}{cc}
0 & -i \\
i & 0
\end{array}\right), \quad e_{2}=\left(\begin{array}{cc}
0 & -1 \\
1 & 0
\end{array}\right), \quad e_{3}=\left(\begin{array}{cc}
-1 & 0 \\
0 & 1
\end{array}\right) .
$$

Существует следующее ортогональное представление $S U(2)$ на $\mathbb{R}^{3}$ :

$$
\begin{aligned}
e_{k} & \rightarrow \rho(S)\left(e_{k}\right)=\bar{S}^{\top} e_{i} S=S^{*} e_{k} S, \quad k=1,2,3, \\
S & =\left(\begin{array}{cc}
\lambda & \mu \\
-\bar{\mu} & \bar{\lambda}
\end{array}\right) \in S U(2), \quad \text { т.e. } \quad|\lambda|^{2}+|\mu|^{2}=1,
\end{aligned}
$$

которое пропускается через $S O(3)=S U(2) /\{ \pm 1\}$. Следующая лемма доказывается прямыми вычислениями.

Лемма 1. Если поверхность $\Sigma$ задана вектор-функиией $\psi$ посредством представления Вейерштрасса, то

1) $\lambda \psi+\mu \psi^{*}$ задает поверхность, полученную из $\Sigma$ преобразованием $\rho(S)$ обгемлющего пространства $\mathbb{R}^{3}$;

2) $\lambda \psi$ с $\lambda \in \mathbb{R}$ задает образ поверхности $\Sigma$ под действием гомотетии $x \rightarrow \lambda x$.

ЗАмЕчаниЕ. Формулы (1) и (3) были введены в [2] для построения поверхностей, допускающих солитонные деформации, заданные модифицированным уравнением Веселова-Новикова. Они восходят к некоторым комплекснозначным формулам, полученным для других целей Эйзенхартом [16]. Аналогичное представление для поверхностей постоянной средней кривизны в терминах оператора Дирака было предложено Абрешем в 1989 г. (доклад в Люмини). Достаточно скоро было понято, что эти формулы дают локальное представление 
произвольной поверхности (см. [1]; в доказательстве, изложенном выше, мы следовали работе [10], позднее другое доказательство было дано в [17] и с физической точки представление было описано в [18]). Более того, это представление оказалось эквивалентным представлению Кенмоцу [19], которое не было связано явно с оператором Дирака.

2.2. Глобальное представление Вейерштрасса. В [1] было введено глобальное представление Вейерштрасса замкнутых поверхностей. Именно для этого необходимо использовать специальные $\psi_{1}$-расслоения над поверхностями и рассматривать оператор Дирака, определенный на сечениях таких расслоений. При этом

а) функционал Уиллмора возникает как интеграл от квадрата нормы потенциала $U$ и конформная геометрия поверхности связывается со спектральными свойствами соответствующего оператора Дирака;

б) так как в [1] было доказано, что торы деформируются в торы под действием потока, заданного модифицированным уравнением Веселова-Новикова, и функционал Уиллмора сохраняется этим потоком, пространство модулей погруженных торов вкладывается в фазовое пространство интегрируемой системы, для которой функционал Уиллмора является интегралом движения.

Согласно теореме об униформизации каждая замкнутая ориентированная поверхность $\Sigma$ конформно эквивалентна поверхности постоянной секционной кривизны $\Sigma_{0}$ и выбор конформного параметра $z$ на $\Sigma$ задает такую эквивалентность $\Sigma_{0} \rightarrow \Sigma$.

Поскольку величины

$$
\bar{\psi}_{2}^{2} d z, \quad \psi_{1}^{2} d z, \quad \psi_{1} \bar{\psi}_{2} d z, \quad e^{2 \alpha} d z d \bar{z}, \quad H=2 U e^{-\alpha}
$$

определены глобально на поверхности $\Sigma_{0}$, это приводит к следующему описанию:

ТЕорема 2 [1], [6]. Любая ориентированная замкнутая поверхность $\Sigma$, погруженная в $\mathbb{R}^{3}$, имеет представление Вейерштрасса вида $(2),(3)$, где $\psi$ является сечением некоторого расслоения Е над конформно эквивалентной поверхностью $\Sigma_{0}$, имеющей постоянную секционную кривизну, и $\mathscr{D} \psi=0$. При этом

1) если $\Sigma=\mathbb{C} \cup\{\infty\}$ - сфера, то $\psi$ и $U$, определенные на $\mathbb{C}$, распространяются на окрестность бесконечности по формулам

$$
\left(\psi_{1}, \bar{\psi}_{2}\right) \rightarrow\left(z \psi_{1}, z \bar{\psi}_{2}\right), \quad U \rightarrow|z|^{2} U \quad \text { при } \quad z \rightarrow-z^{-1},
$$

и U имеет следующую асимптотику на бесконечности:

$$
U=\frac{\text { const }}{|z|^{2}}+O\left(\frac{1}{|z|^{3}}\right) \quad \text { при } \quad z \rightarrow \infty ;
$$

2) если $\Sigma$ конформно эквивалентна тору $\Sigma_{0}=\mathbb{R}^{2} / \Lambda$, то

$U(z+\gamma, \bar{z}+\bar{\gamma})=U(z, \bar{z}), \quad \psi(z+\gamma, \bar{z}+\bar{\gamma})=\mu(\gamma) \psi(z, \bar{z}) \quad$ для всех $\quad \gamma \in \Lambda$, где $\mu \in\{ \pm 1\}$ - характер группы $\Lambda \rightarrow\{ \pm 1\}$, принимающий значения в $\{ \pm 1\}$ и задающий расслоение

$$
E \stackrel{\mathbb{C}^{2}}{\longrightarrow} \Sigma_{0}
$$


сечением которого является вектор-функиия $\psi=\left(\psi_{1}, \psi_{2}\right)^{\perp}$;

3) если $\Sigma$ - поверхность рода $g \geqslant 2$, то $\Sigma_{0}=\mathscr{H} / \Lambda$, где $\mathscr{H}$ - верхняя полуплоскость Лобачевского и $\Lambda$ - дискретная подгруппа в $\operatorname{PSL}(2, \mathbb{R})$, которая действует на $\mathscr{H}=\{\operatorname{Im} z>0\} \subset \mathbb{C}$ по формуле

$$
z \rightarrow \gamma(z)=\frac{a z+b}{c z+d}, \quad\left(\begin{array}{ll}
a & b \\
c & d
\end{array}\right) \in S L(2, \mathbb{R})
$$

При этом $\psi$-расслоение

$$
E \stackrel{\mathbb{C}^{2}}{\longrightarrow} \Sigma_{0}
$$

задается правилами монодромии

$$
\gamma:\left(\psi_{1}, \bar{\psi}_{2}\right) \rightarrow(c z+d)\left(\psi_{1}, \bar{\psi}_{2}\right)
$$

$u$

$$
U(\gamma(z), \overline{\gamma(z)})=|c z+d|^{2} U(z, \bar{z})
$$

Расслоение Е расщепляется в сумму двух сопряженных расслоений $E=$ $E_{0} \oplus \bar{E}_{0}$, чвими сечениями являются $\psi_{1}$ и $\psi_{2}$ соответственно.

Поскольку $P S L(2, \mathbb{R})=S L(2, \mathbb{R}) /\{ \pm 1\}$, элемент $\gamma \in P S L(2, \mathbb{R})$ определяет монодромию с точностью до знака. Аналогичная ситуация имеет место для торов. Поэтому расслоения $E$ называются спинорными.

При заданном конформном параметре на $\Sigma$ потенциал $U$ фиксирован и задает потенциал представления. Более того,

$$
\mathscr{W}(\Sigma)=4 \int_{\Sigma} U^{2} d x \wedge d y
$$

Каждое сечение $\psi \in \Gamma(E)$ такое, что $\mathscr{D} \psi=0$, задает поверхность, которая в общем случае незамкнута, а только имеет периодическое гауссово отображение. При этом формулы Вейерштрасса задают погружение универсального накрытия $\widetilde{\Sigma}$ поверхности $\Sigma$. Следующее предложение показывает, когда такое погружение факторизуется в погружение компактной поверхности.

ПреДЛОЖЕНИЕ 2. Представление Вейерштрасса задает погружение компактной поверхности $\Sigma$, если и только если

$$
\int_{\Sigma_{0}} \bar{\psi}_{1}^{2} d \bar{z} \wedge \omega=\int_{\Sigma_{0}} \psi_{2}^{2} d \bar{z} \wedge \omega=\int_{\Sigma_{0}} \bar{\psi}_{1} \psi_{2} d \bar{z} \wedge \omega=0
$$

для каждого голоморфного дифференииала $\omega$ на $\Sigma_{0}$.

Мы видим, что каждому погруженному тору $\Sigma \subset \mathbb{R}^{3}$ с заданным конформным параметром $z$ отвечает оператор Дирака $\mathscr{D}$ с двояко-периодическим потенциалом

$$
U=V=\frac{H e^{\alpha}}{2},
$$

где $H$ - средняя кривизна и $e^{2 \alpha} d z d \bar{z}-$ индуцированная метрика. 
2.3. Поверхности в трехмерных группах Ли. Для поверхностей в трехмерных группах Ли представление Вейерштрасса обобщается следующим образом.

Пусть $G$ - трехмерная группа Ли с левоинвариантной метрикой и

$$
f: \Sigma \rightarrow G
$$

- погружение поверхности $\Sigma$ в $G$. Через $\mathscr{G}$ обозначим алгебру Ли группы $G$. Пусть $z=x+i y-$ конформный параметр на поверхности.

Рассмотрим обратный образ касательного расслоения $T G-\mathscr{G}$-расслоение над $\Sigma: \mathscr{G} \rightarrow E=f^{-1}(T G) \stackrel{\pi}{\rightarrow} \Sigma$, и рассмотрим дифференциал

$$
d_{\mathscr{A}}: \Omega^{1}(\Sigma ; E) \rightarrow \Omega^{2}(\Sigma ; E),
$$

который действует на $E$-значных 1-формах:

$$
d_{\mathscr{A}} \omega=d_{\mathscr{A}}^{\prime} \omega+d_{\mathscr{A}}^{\prime \prime} \omega
$$

где $\omega=u d z+u^{*} d \bar{z}$ и

$$
d_{\mathscr{A}}^{\prime} \omega=-\nabla_{\bar{\partial} f} u d z \wedge d \bar{z}, \quad d_{\mathscr{A}}^{\prime \prime} \omega=\nabla_{\partial f} u^{*} d z \wedge d \bar{z} .
$$

Прямыми вычислениями мы выводим первое деривационное уравнение

$$
d_{\mathscr{A}}(d f)=0 .
$$

Вектор напряжения $\tau(f)$ определяется из уравнения

$$
d_{\mathscr{A}}(* d f)=f \cdot\left(e^{2 \alpha} \tau(f)\right) d x \wedge d y=\frac{i}{2} f \cdot\left(e^{2 \alpha} \tau(f)\right) d z \wedge d \bar{z}
$$

где $f \cdot \tau(f)=2 H N, N$ - нормаль и $H$ - средняя кривизна. Это приводит ко второму деривационному уравнению:

$$
d_{\mathscr{A}}(* d f)=i e^{2 \alpha} H N d z \wedge d \bar{z} .
$$

Поскольку метрика левоинвариантная, мы перепишем деривационные уравнения в терминах

$$
\Psi=f^{-1} \partial f, \quad \Psi^{*}=f^{-1} \bar{\partial} f
$$

следующим образом:

$$
\begin{gathered}
\partial \Psi^{*}-\bar{\partial} \Psi+\nabla_{\Psi} \Psi^{*}-\nabla_{\Psi^{*}} \Psi=0, \\
\partial \Psi^{*}+\bar{\partial} \Psi+\nabla_{\Psi} \Psi^{*}+\nabla_{\Psi^{*}} \Psi=e^{2 \alpha} H f^{-1}(N) .
\end{gathered}
$$

Уравнение (10) эквивалентно уравнению (8), а уравнение (11) - уравнению (9).

Возьмем ортонормированный базис $e_{1}, e_{2}, e_{3}$ в алгебре Ли $\mathscr{G}$ группы $G$ и разложим $\Psi$ и $\Psi^{*}$ по этому базису:

$$
\Psi=\sum_{k=1}^{3} Z_{k} e_{k}, \quad \Psi^{*}=\sum_{k=1}^{3} \bar{Z}_{k} e_{k} .
$$


Уравнения (10) и (11) принимают вид

$$
\begin{gathered}
\sum_{j}\left(\partial \bar{Z}_{j}-\bar{\partial} Z_{j}\right) e_{j}+\sum_{j, k}\left(Z_{j} \bar{Z}_{k}-\bar{Z}_{j} Z_{k}\right) \nabla_{e_{j}} e_{k}=0 \\
\sum_{j}\left(\partial \bar{Z}_{j}+\bar{\partial} Z_{j}\right) e_{j}+\sum_{j, k}\left(Z_{j} \bar{Z}_{k}+\bar{Z}_{j} Z_{k}\right) \nabla_{e_{j}} e_{k} \\
=2 i H\left[\left(\bar{Z}_{2} Z_{3}-Z_{2} \bar{Z}_{3}\right) e_{1}+\left(\bar{Z}_{3} Z_{1}-Z_{3} \bar{Z}_{1}\right) e_{2}+\left(\bar{Z}_{1} Z_{2}-Z_{1} \bar{Z}_{2}\right) e_{3}\right] .
\end{gathered}
$$

Здесь мы подразумеваем, что базис $\left\{e_{1}, e_{2}, e_{3}\right\}$ положительно ориентирован и, следовательно,

$$
f^{-1}(N)=2 i e^{-2 \alpha}\left[\left(\bar{Z}_{2} Z_{3}-Z_{2} \bar{Z}_{3}\right) e_{1}+\left(\bar{Z}_{3} Z_{1}-Z_{3} \bar{Z}_{1}\right) e_{2}+\left(\bar{Z}_{1} Z_{2}-Z_{1} \bar{Z}_{2}\right) e_{3}\right]
$$

(для $G=S U(2)$ с метрикой Киллинга эта формула принимает вид $f^{-1}(N)=$ $\left.2 i e^{-2 \alpha}\left[\Psi^{*}, \Psi\right]\right)$. Так как параметр $z$ конформный, мы имеем

$$
\langle\Psi, \Psi\rangle=\left\langle\Psi^{*}, \Psi^{*}\right\rangle=0, \quad\left\langle\Psi, \Psi^{*}\right\rangle=\frac{1}{2} e^{2 \alpha},
$$

что переписывается как

$$
Z_{1}^{2}+Z_{2}^{2}+Z_{3}^{2}=0, \quad\left|Z_{1}\right|^{2}+\left|Z_{2}\right|^{2}+\left|Z_{3}\right|^{2}=\frac{1}{2} e^{2 \alpha}
$$

Следовательно, как и в случае поверхностей в $\mathbb{R}^{3}$, вектор $Z$ параметризуется в терминах $\psi$ следующим образом:

$$
Z_{1}=\frac{i}{2}\left(\bar{\psi}_{2}^{2}+\psi_{1}^{2}\right), \quad Z_{2}=\frac{1}{2}\left(\bar{\psi}_{2}^{2}-\psi_{1}^{2}\right), \quad Z_{3}=\psi_{1} \bar{\psi}_{2} .
$$

Покажем, как восстановить поверхность по вектор-функции $\psi$, удовлетворяющей деривационным уравнениям (10)) и (11). В случае некоммутативных групп Ли это нельзя сделать посредством интегральных формул Вейерштрасса.

Пусть $\psi$ задана на поверхности $\Sigma$ с комплексным параметром $z$ и $\Psi$ построена по $\psi$ и удовлетворяет уравнениям (10) и (11). Возьмем точку $P \in M$. Подставим $\psi$ в формулу (14) для компонент $Z_{1}, Z_{2}, Z_{3}$ вектора $\Psi=\sum_{k=1}^{3} Z_{k} e_{k}=$ $f^{-1} \partial f$ и решим линейное уравнение в группе $G$ :

$$
f_{z}=f \Psi
$$

с начальными данными $f(P)=g \in G$. Таким образом мы получим поверхность как отображение

$$
f: \Sigma \rightarrow G
$$

Для группы $\mathbb{R}^{3}$ решение этого уравнения дается формулами Вейерштрасса (2) и (3).

Из вывода уравнений (10) и (11) ясно, что каждая поверхность $\Sigma$ в $G$ получается с помощью этой процедуры, которая является обобщенным представлением Вейерштрасса для поверхностей в группах Ли. При этом мы говорим, что $\psi$ порождает поверхность $\Sigma$. 
Запишем деривационные уравнения (10) и (11) в терминах $\psi$. Они записываются как уравнение Дирака

$$
\mathscr{D} \psi=\left[\left(\begin{array}{cc}
0 & \partial \\
-\bar{\partial} & 0
\end{array}\right)+\left(\begin{array}{cc}
U & 0 \\
0 & V
\end{array}\right)\right] \psi=0,
$$

индуцированная метрика дается той же формулой

$$
d s^{2}=e^{2 \alpha} d z d \bar{z}, \quad e^{\alpha}=\left|\psi_{1}\right|^{2}+\left|\psi_{2}\right|^{2},
$$

и дифференциал Хопфа $A d z^{2}$ равен

$$
A=\left(\bar{\psi}_{2} \partial \psi_{1}-\psi_{1} \partial \bar{\psi}_{2}\right)+\left(\sum_{j, k} Z_{j} Z_{k} \nabla_{e_{j}} e_{k}, N\right)
$$

Для компактной группы с метрикой Киллинга и, в частности, для $G=S U(2)$ мы имеем $\nabla_{e_{j}} e_{k}=-\nabla_{e_{k}} e_{j}$ и дифференциал Хопфа принимает тот же вид, что и для поверхностей в $\mathbb{R}^{3}: A=\bar{\psi}_{2} \partial \psi_{1}-\psi_{1} \partial \bar{\psi}_{2}$.

Мы рассмотрим трехмерные группы Ли с геометриями Тёрстона. Напомним, что, согласно теореме Тёрстона [20], [21], все трехмерные максимальные односвязные геометрии $(X$, Isom $X)$, допускающие компактные факторпространства, даются следующим списком:

1) геометрии постоянной секционной кривизны: $X=\mathbb{R}^{3}, S^{3}$ и $H^{3}$;

2) геометрии произведений: $X=S^{2} \times \mathbb{R}$ и $H^{2} \times \mathbb{R}$;

3) три геометрии на группах Ли Nil, Sol и $\widetilde{S L}_{2}$ с некоторыми левоинвариантными метриками.

Группа $\mathbb{R}^{3}$ с евклидовой метрикой уже была рассмотрена выше. Нам остаются четыре группы:

$$
S U(2)=S^{3}, \quad \text { Nil, } \quad \text { Sol, } \quad \widetilde{S L}_{2},
$$

где Nil - нильпотентная группа, Sol - разрешимая группа, а $\widetilde{S L}{ }_{2}-$ универсальное накрытие группы $S L_{2}(\mathbb{R})$ :

$$
\mathrm{Nil}=\left\{\left(\begin{array}{ccc}
1 & x & z \\
0 & 1 & y \\
0 & 0 & 1
\end{array}\right)\right\}, \quad \operatorname{Sol}=\left\{\left(\begin{array}{ccc}
e^{-z} & 0 & x \\
0 & e^{z} & y \\
0 & 0 & 1
\end{array}\right)\right\}
$$

где $x, y, z \in \mathbb{R}$.

Случай $G=S U(2)$ был изучен в [10] и поверхности в других группах были рассмотрены в [11]:

a) $G=S U(2)$ :

$$
U=\bar{V}=\frac{1}{2}(H-i)\left(\left|\psi_{1}\right|^{2}+\left|\psi_{2}\right|^{2}\right),
$$

уравнения Гаусса-Вейнгартена:

$$
\left[\frac{\partial}{\partial z}-\left(\begin{array}{cc}
\alpha_{z} & A e^{-\alpha} \\
-U & 0
\end{array}\right)\right] \psi=\left[\frac{\partial}{\partial \bar{z}}-\left(\begin{array}{cc}
0 & \bar{U} \\
-\bar{A} e^{-\alpha} & \alpha_{\bar{z}}
\end{array}\right)\right] \psi=0
$$

их условия совместности - уравнения Гаусса-Кодацци - имеют вид

$$
\alpha_{z \bar{z}}+|U|^{2}-|A|^{2} e^{-2 \alpha}=0, \quad A_{\bar{z}}=\left(\bar{U}_{z}-\alpha_{z} \bar{U}\right) e^{\alpha},
$$


где $A d z^{2}$ - дифференциал Хопфа:

$$
A=\bar{\psi}_{2} \partial \psi_{1}-\psi_{1} \partial \bar{\psi}_{2}
$$

б) $G=\mathrm{Nil}$ :

$$
U=V=\frac{H}{2}\left(\left|\psi_{1}\right|^{2}+\left|\psi_{2}\right|^{2}\right)+\frac{i}{4}\left(\left|\psi_{2}\right|^{2}-\left|\psi_{1}\right|^{2}\right),
$$

уравнения Гаусса-Вейнгартена имеют вид

$$
\begin{gathered}
{\left[\frac{\partial}{\partial z}-\left(\begin{array}{cc}
\alpha_{z}-\frac{i}{2} \psi_{1} \bar{\psi}_{2} & A e^{-\alpha} \\
-U & 0
\end{array}\right)\right] \psi} \\
{\left[\frac{\partial}{\partial \bar{z}}-\left(\begin{array}{cc}
0 & U \\
-\bar{A} e^{-\alpha} & \alpha_{\bar{z}}-\frac{i}{2} \bar{\psi}_{1} \psi_{2}
\end{array}\right)\right] \psi}
\end{gathered}
$$

а уравнения Гаусса-Кодацци -

$$
\begin{gathered}
\alpha_{z \bar{z}}-|A|^{2} e^{-2 \alpha}+\frac{H^{2}}{4} e^{2 \alpha}=\frac{1}{16}\left(3\left|\psi_{1}\right|^{4}+3\left|\psi_{2}\right|^{4}-10\left|\psi_{1}\right|^{2}\left|\psi_{2}\right|^{2}\right), \\
A_{\bar{z}}-\frac{H_{z}}{2} e^{2 \alpha}+\frac{1}{2}\left(\left|\psi_{2}\right|^{4}-\left|\psi_{1}\right|^{4}\right) \psi_{1} \bar{\psi}_{2}=0
\end{gathered}
$$

где дифференциал Хопфа равен

$$
A=\left(\bar{\psi}_{2} \partial \psi_{1}-\psi_{1} \partial \bar{\psi}_{2}\right)+i \psi_{1}^{2} \bar{\psi}_{2}^{2}
$$

в) $G=\widetilde{S L}_{2}$ :

$$
\begin{aligned}
& U=\frac{H}{2}\left(\left|\psi_{1}\right|^{2}+\left|\psi_{2}\right|^{2}\right)+i\left(\frac{1}{2}\left|\psi_{1}\right|^{2}-\frac{3}{4}\left|\psi_{2}\right|^{2}\right), \\
& V=\frac{H}{2}\left(\left|\psi_{1}\right|^{2}+\left|\psi_{2}\right|^{2}\right)+i\left(\frac{3}{4}\left|\psi_{1}\right|^{2}-\frac{1}{2}\left|\psi_{2}\right|^{2}\right),
\end{aligned}
$$

уравнения Гаусса-Вейнгартена записываются как

$$
\begin{aligned}
& {\left[\frac{\partial}{\partial z}-\left(\begin{array}{cc}
\alpha_{z}+\frac{5 i}{4} \psi_{1} \bar{\psi}_{2} & A e^{-\alpha} \\
-U & 0
\end{array}\right)\right] \psi=0,}
\end{aligned}
$$

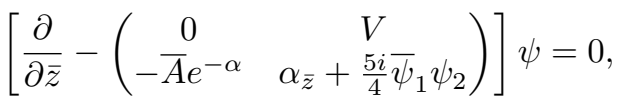

уравнения Гаусса-Кодацци имеют вид

$$
\begin{gathered}
\alpha_{z \bar{z}}-e^{-2 \alpha}|A|^{2}+\frac{1}{4} e^{2 \alpha} H^{2}=e^{2 \alpha}-5\left|Z_{3}\right|^{2}, \\
\bar{\partial}\left(A+\frac{5 Z_{3}^{2}}{2(H-i)}\right)=\frac{1}{2} H_{z} e^{2 \alpha}+\bar{\partial}\left(\frac{5}{2(H-i)}\right) Z_{3}{ }^{2},
\end{gathered}
$$

где дифференциал Хопфа равен

$$
A=\left(\bar{\psi}_{2} \partial \psi_{1}-\psi_{1} \partial \bar{\psi}_{2}\right)-\frac{5 i}{2} \psi_{1}^{2} \bar{\psi}_{2}^{2}
$$


г) $G=$ Sol: мы рассматриваем только области, где $Z_{3}=\psi_{1} \bar{\psi}_{2}$, для которых

$$
\begin{aligned}
& U=\frac{H}{2}\left(\left|\psi_{1}\right|^{2}+\left|\psi_{2}\right|^{2}\right)+\frac{1}{2} \bar{\psi}_{2}^{2} \frac{\bar{\psi}_{1}}{\psi_{1}}, \\
& V=\frac{H}{2}\left(\left|\psi_{1}\right|^{2}+\left|\psi_{2}\right|^{2}\right)+\frac{1}{2} \bar{\psi}_{1}^{2} \frac{\bar{\psi}_{2}}{\psi_{2}},
\end{aligned}
$$

уравнения Гаусса-Вейнгартена состоят из уравнения Дирака и следующей системы уравнений:

$$
\partial \psi_{1}=\alpha_{z} \psi_{1}+A e^{-\alpha} \psi_{2}-\frac{1}{2} \bar{\psi}_{2}^{3}, \quad \bar{\partial} \psi_{2}=-\bar{A} e^{-\alpha} \psi_{1}+\alpha_{\bar{z}} \psi_{2}-\frac{1}{2} \bar{\psi}_{1}^{3},
$$

уравнения Гаусса-Кодацци принимают вид

$$
\begin{aligned}
\alpha_{z \bar{z}}-e^{-2 \alpha}|A|^{2}+\frac{1}{4} e^{2 \alpha} H^{2} & =\frac{1}{4}\left(6\left|\psi_{1}\right|^{2}\left|\psi_{2}\right|^{2}-\left(\left|\psi_{1}\right|^{4}+\left|\psi_{2}\right|^{4}\right)\right), \\
A_{\bar{z}}-\frac{1}{2} H_{z} e^{2 \alpha} & =\left(\left|\psi_{2}\right|^{4}-\left|\psi_{1}\right|^{4}\right) \psi_{1} \bar{\psi}_{2}
\end{aligned}
$$

где

$$
A=\left(\bar{\psi}_{2} \partial \psi_{1}-\psi_{1} \partial \bar{\psi}_{2}\right)+\frac{1}{2}\left(\bar{\psi}_{2}^{4}-\psi_{1}^{4}\right) .
$$

Необходимо сделать несколько объясняющих замечаний.

1) Для последних трех групп в формулах возникает выражение $Z_{3}$. Направление вектора $e_{3}$ имеет различный смысл для этих групп:

1a) группы Nil и $\widetilde{S L}_{2}$ допускают $S^{1}$-симметрии, являющиеся вращениями вокруг геодезической, выпущенной в направлении вектора $e_{3}$; это вращение вместе с левыми сдвигами порождает группу изометрий Isom $G$;

1б) для группы Sol векторы $e_{1}$ и $e_{2}$ коммутируют. Следовательно, уравнение $Z_{3}=\psi_{1} \bar{\psi}_{2}=0$ может выполняться на открытом подмножестве $B$ поверхности и уравнение Дирака не продолжается по непрерывности на всю поверхность. Так как $H=0$ в $B$, мы полагаем

$$
U=V=0 \quad \text { при } \quad \psi_{1} \bar{\psi}_{2}=0 \quad \text { и } \quad G=\text { Sol . }
$$

Но на границе $\partial B$ множества $\left\{Z_{3} \neq 0\right\}$ потенциалы $U_{\text {Sol }}$ и $V_{\text {Sol }}$ не всегда корректно определены из-за неопределенности выражения $\frac{\bar{\psi}_{1}}{\psi_{1}}$ при $\psi_{1}=0$, и уравнение Дирака с данными потенциалами выполняется вне $\partial B$.

2) При $G=\mathbb{R}^{3}$ или $S U(2)$ уравнения Гаусса-Кодацци выводятся следующим образом. Мы имеем

$$
R \psi=(\partial-\mathscr{A})(\bar{\partial}-\mathscr{B}) \psi-(\bar{\partial}-\mathscr{B})(\partial-\mathscr{A}) \psi=\left(\mathscr{A}_{\bar{z}}-\mathscr{B}_{z}+[\mathscr{A}, \mathscr{B}]\right) \psi=0,
$$

где $(\partial-\mathscr{A}) \psi=(\bar{\partial}-\mathscr{B}) \psi=0$ - это уравнения Гаусса-Вейнгартена и векторфункция $\psi^{*}$ (см. (4)) удовлетворяет тому же самому уравнению $R \psi^{*}=0$, что вместе с $R \psi=0$ влечет $R=\mathscr{A}_{\bar{z}}-\mathscr{B}_{z}+[\mathscr{A}, \mathscr{B}]=0$. Для других групп уравнения $\mathscr{D} \psi^{*}=0$ и $R \psi^{*}=0$ не выполняются и, в частности, ядро оператора Дирака не может рассматриваться как векторное пространство над кватернионами. Поэтому уравнения Гаусса-Кодацци выведены в [11] другими методами. 
3) В действительности уравнения Дирака в случае некоммутативных групп Ли нелинейны по $\psi$ из-за ограничений на потенциалы. Следовательно, если $\psi$ определяет поверхность, то $\lambda \psi$ не задает другую поверхность при $|\lambda| \neq 1$, поскольку эти группы не допускают гомотетий. Для $S U(2)$ отображение (4) переводит решение уравнения Дирака в другое решение этого уравнения и выполняется аналог части 1) леммы 1: $\lambda \psi+\mu \psi^{*},|\lambda|^{2}+|\mu|^{2}=1$, задает образ изначальной поверхности при внутреннем автоморфизме группы $S U(2)$, отвечающем вращению алгебры Ли.

Укажем несколько следствий. Поскольку случай $G=S U(2)$ был хорошо изучен, ${ }^{3}$ мы рассмотрим только другие группы.

ТЕОРема 3. 1) Если $\psi$ порождает минимальную поверхность в трехмерной группе Ли, то выполняются следующие уравнения:

$$
\begin{aligned}
& \bar{\partial} \psi_{1}=\frac{i}{4}\left(\left|\psi_{2}\right|^{2}-\left|\psi_{1}\right|^{2}\right) \psi_{2}, \quad \partial \psi_{2}=-\frac{i}{4}\left(\left|\psi_{2}\right|^{2}-\left|\psi_{1}\right|^{2}\right) \psi_{1} \quad \text { при } \quad G=\mathrm{Nil}, \\
& \bar{\partial} \psi_{1}=i\left(\frac{3}{4}\left|\psi_{1}\right|^{2}-\frac{1}{2}\left|\psi_{2}\right|^{2}\right) \psi_{2}, \quad \partial \psi_{2}=-i\left(\frac{1}{2}\left|\psi_{1}\right|^{2}-\frac{3}{4}\left|\psi_{2}\right|^{2}\right) \psi_{1} \quad \text { при } \quad G=\widetilde{S L}_{2} \text {, } \\
& \bar{\partial} \psi_{1}=\frac{1}{2} \bar{\psi}_{1}^{2} \bar{\psi}_{2}, \quad \partial \psi_{2}=-\frac{1}{2} \bar{\psi}_{1} \bar{\psi}_{2}^{2} \quad \text { при } \quad G=\text { Sol . }
\end{aligned}
$$

2) (Абреш [22]) Если поверхность имеет постоянную среднюю кривизну, то квадратичный дифференциал $\widetilde{A} d z^{2}$ голоморфен:

$$
\begin{array}{ll}
\widetilde{A} d z^{2}=\left(A+\frac{Z_{3}^{2}}{2 H+i}\right) d z^{2} \quad \text { при } \quad G=\mathrm{Nil}, \\
\widetilde{A} d z^{2}=\left(A+\frac{5}{2(H-i)} Z_{3}^{2}\right) d z^{2} \quad \text { при } \quad G=\widetilde{S L}_{2} .
\end{array}
$$

3) Если для поверхности в $G=\mathrm{Nil}$ дифференциал $\widetilde{A} d z^{2}$ голоморфен, то она имеет постоянную среднюю кривизну.

Было бы интересно понять связь формул для поверхностей постоянной средней кривизны с солитонными уравнениями. Такие связи известны для поверхностей в $\mathbb{R}^{3}$ и $S U(2)$.

Аналоги утверждения 2) известны для поверхностей в $S^{2} \times \mathbb{R}$ и $H^{2} \times \mathbb{R}[23]$. Но только для поверхностей в Nil обратное утверждение 3) тоже доказано. ${ }^{4}$

Мы заметим, что для минимальных поверхностей в Nil и Sol аналог представления Вейерштрасса был получен другими методами в [25], [26]. Другие подходы к изучению поверхностей в группах Ли были применены в [27], [28].

${ }^{3}$ Для $S U(2)$ уравнения минимальных поверхностей имеют вид

$$
\bar{\partial} \psi_{1}=-\frac{i}{2}\left(\left|\psi_{1}\right|^{2}+\left|\psi_{2}\right|^{2}\right) \psi_{2}, \quad \partial \psi_{2}=\frac{i}{2}\left(\left|\psi_{1}\right|^{2}+\left|\psi_{2}\right|^{2}\right) \psi_{1},
$$

и поверхности постоянной средней кривизны выделяются условием $A_{\bar{z}}=0$.

${ }^{4}$ После того как эта статья была представлена к печати, было доказано, что для поверхностей в $\widetilde{S L}_{2}$ и $H^{2} \times \mathbb{R}$ аналогичное утверждение неверно: существуют поверхности, для которых квадратичный дифференциал $\widetilde{A} d z^{2}$ голоморфен, но их средняя кривизна не постоянна [24]. 
2.4. Кватернионный язык и кватернионная теория функций. Педит и Пинкалль записали представление Вейерштрасса для поверхностей в $\mathbb{R}^{3}$ на языке кватернионов и затем распространили его на поверхности в $\mathbb{R}^{4}[8]$ (см. некоторые предварительные результаты в [29]-[31]).

Действительно, идея использования кватернионов исходит из симметрии ядра оператора Дирака относительно преобразования (4) (заметим, что это выполняется для поверхностей в $\mathbb{R}^{3}$ и $S U(2)$, когда $U=\bar{V}$, и не верно для поверхностей в других трехмерных группах Ли).

Идентифицируем $\mathbb{C}^{2}$ с пространством кватернионов $\mathbb{H}$ следующим образом:

$$
\left(z_{1}, z_{2}\right) \rightarrow z_{1}+\mathbf{j} z_{2}=\left(\begin{array}{cc}
z_{1} & -\bar{z}_{2} \\
z_{2} & \bar{z}_{1}
\end{array}\right)
$$

и рассмотрим два матричных оператора

$$
\bar{\partial}=\left(\begin{array}{ll}
\bar{\partial} & 0 \\
0 & \partial
\end{array}\right), \quad \mathbf{j} U=\mathbf{j}\left(\begin{array}{cc}
U & 0 \\
0 & \bar{U}
\end{array}\right)=\left(\begin{array}{cc}
0 & -\bar{U} \\
U & 0
\end{array}\right) .
$$

Здесь $\mathbf{j}$ - одна из стандартных образующих алгебры кватернионов, и мы имеем

$$
\mathbf{j}^{2}=-1, \quad z \mathbf{j}=\mathbf{j} \bar{z}, \quad \bar{\partial} \mathbf{j}=\mathbf{j} \partial .
$$

Уравнение Дирака принимает вид

$$
(\bar{\partial}+\mathbf{j} U)\left(\psi_{1}+\mathbf{j} \psi_{2}\right)=\left(\bar{\partial} \psi_{1}-\bar{U} \psi_{2}\right)+\mathbf{j}\left(\partial \psi_{2}+U \psi_{1}\right)=0 .
$$

Поскольку, согласно $(6), \psi_{1}$ и $\bar{\psi}_{2}$ - сечения одного и того же расслоения $E_{0}$, в терминах кватернионов разумнее переписать уравнение Дирака в виде

$$
(\bar{\partial}+\mathbf{j} U)\left(\psi_{1}+\bar{\psi}_{2} \mathbf{j}\right)=0 .
$$

Можно рассматривать $L=E_{0} \oplus E_{0}$ как кватернионное линейное расслоение, чьи сечения имеют вид $\psi_{1}+\bar{\psi}_{2} \mathbf{j}$ и которое снабжено таким кватернионно линейным эндоморфизмом $J$, что $J^{2}=-1$. В нашем случае $J$ действует просто как умножение справа на $\mathbf{j}$ :

$$
J:\left(\psi_{1}, \bar{\psi}_{2}\right) \rightarrow\left(-\bar{\psi}_{2}, \bar{\psi}_{1}\right) \quad \text { или } \quad \psi_{1}+\bar{\psi}_{2} \mathbf{j} \rightarrow\left(\psi_{1}+\bar{\psi}_{2} \mathbf{j}\right) \mathbf{j}=-\overline{\psi_{2}}+\psi_{1} \mathbf{j} .
$$

Отображение $J$ определяет для каждого кватернионного слоя каноническое разбиение в сумму $\mathbb{C} \oplus \mathbb{C}$ (в нашем случае - разбиение на слагаемые $\psi_{1}$ и $\bar{\psi}_{2}$ ). В [8], [12] такое расслоение называется "комплексным кватернионным линейным расслоением".

Оператор Дирака в этих терминах есть

$$
\mathscr{D} \psi=(\bar{\partial}+\mathbf{j} U)\left(\psi_{1}+\bar{\psi}_{2} \mathbf{j}\right)=\left(\bar{\partial} \psi_{1}-\bar{U} \psi_{2}\right)+\left(\overline{\partial \psi}_{2}+\bar{U}_{1}\right) \mathbf{j},
$$

и мы видим, что его ядро инвариантно умножениям справа на постоянные кватернионы (см. лемму 1) и, следовательно, ядро может быть рассмотрено как векторное пространство над кватернионами $\mathbb{H}$. Согласно (6), мы имеем оператор

$$
\mathscr{D}: \Gamma(L) \rightarrow \Gamma(\bar{K} L)
$$

где для расслоения $V$ мы обозначаем через $\Gamma(V)$ пространство его сечений и $\bar{K}$ - пучок 1 -форм типа $(0,1)$, т.е. вида $f d \bar{z}$, над поверхностью $\Sigma_{0}$. 
Этот оператор, конечно, не является линейным относительно умножений справа на кватернионнозначные функции, и выполняется следующая очевидная формула:

$$
\mathscr{D}(\psi \lambda)=(\mathscr{D} \psi) \lambda+\psi_{1}(\bar{\mu}+\mathbf{j} \partial \eta)+\bar{\psi}_{2}(-\bar{\partial} \eta+\mathbf{j} \partial \mu)
$$

где $\lambda=\mu+\mathbf{j} \eta=\mu+\bar{\eta} \mathbf{j}$. В [8] эта формула выписана в бескоординатной форме как

$$
\mathscr{D}(\psi \lambda)=(\mathscr{D} \psi) \lambda+\frac{1}{2}(\psi d \lambda+J \psi * d \lambda),
$$

потенциал $U$, умноженный слева на $\mathbf{j}$, называется полем Хопфа $Q=\mathbf{j} U$ связности $\mathscr{D}$ на $L$, и величина

$$
\mathscr{W}=\int_{\Sigma_{0}}|U|^{2} d x \wedge d y
$$

называется энергией Уиллмора связности $\mathscr{D}$.

Хотя вначале этот кватернионный язык казался очень искусственным, по крайней мере нам, он привел к распространению представления Вейерштрасса на поверхности в $\mathbb{R}^{4}[8]$.

Позднее он был развит в инструмент исследования, состоящий в разработке аналогий между комплексной алгебраической геометрией и теорией комплексных кватернионных линейных расслоений. Оказалось, что он эффективно применим к изучению специальных типов поверхностей и преобразований Бэклунда в рамках конформного подхода, когда не различаются $\mathbb{R}^{4}$ и $S^{4}$ [12], [32]. В конечном итоге этот подход привел к замечательному распространению соотношений типа Плюккера из комплексной алгебраической геометрии на геометрию комплексных кватернионных линейных расслоений и применению их к получению нижних оценок для функционала Уиллмора [7] (см. п. 5.4). При этом эта теория работает с общими расслоениями $L$, не всегда приходящими из теории поверхностей [7]. Расслоения, связанные с поверхностями, выделяются их степенями: из (5) и (6) следует, что

$$
\operatorname{deg} E_{0}=\operatorname{genus}\left(\Sigma_{0}\right)-1=g-1 .
$$

2.5. Поверхности в $\mathbb{R}^{4}$. Грассманиан ориентированных двумерных плоскостей в $\mathbb{R}^{4}$ диффеоморфен квадрике

$$
y_{1}^{2}+y_{2}^{2}+y_{3}^{2}+y_{4}^{2}=0, \quad y \in \mathbb{C} P^{3} .
$$

Рассмотрим другие координаты $y_{1}^{\prime}, y_{2}^{\prime}, y_{3}^{\prime}, y_{4}^{\prime}$ в $\mathbb{C}^{4}$ :

$$
y_{1}=\frac{i}{2}\left(y_{1}^{\prime}+y_{2}^{\prime}\right), \quad y_{2}=\frac{1}{2}\left(y_{1}^{\prime}-y_{2}^{\prime}\right), \quad y_{3}=\frac{1}{2}\left(y_{3}^{\prime}+y_{4}^{\prime}\right), \quad y_{4}=\frac{i}{2}\left(y_{3}^{\prime}-y_{4}^{\prime}\right) .
$$

В этих координатах многообразие $\widetilde{G}_{4,2}$ задается уравнением

$$
y_{1}^{\prime} y_{2}^{\prime}=y_{3}^{\prime} y_{4}^{\prime} .
$$

Очевидно, что существует диффеоморфизм

$$
\mathbb{C} P^{1} \times \mathbb{C} P^{1} \rightarrow \widetilde{G}_{4,2},
$$


заданный отображением Сегре

$$
y_{1}^{\prime}=a_{2} b_{2}, \quad y_{2}^{\prime}=a_{1} b_{1}, \quad y_{3}^{\prime}=a_{2} b_{1}, \quad y_{4}^{\prime}=a_{1} b_{2},
$$

где $\left(a_{1}: a_{2}\right)$ и $\left(b_{1}: b_{2}\right)$ - однородные координаты на различных экземплярах $\mathbb{C} P^{1}$.

Параметризуем $x_{z}^{k}, k=1,2,3,4$, в терминах этих координат и положим

$$
a_{1}=\varphi_{1}, \quad a_{2}=\bar{\varphi}_{2}, \quad b_{1}=\psi_{1}, \quad b_{2}=\bar{\psi}_{2} .
$$

В отличие от трехмерной ситуации эта параметризация неединственна даже по модулю умножений на \pm 1 и вектор-функции $\psi$ и $\varphi$ определены с точностью до калибровочных преобразований

$$
\left(\begin{array}{l}
\psi_{1} \\
\psi_{2}
\end{array}\right) \rightarrow\left(\begin{array}{c}
e^{f} \psi_{1} \\
e^{\bar{f}} \psi_{2}
\end{array}\right), \quad\left(\begin{array}{l}
\varphi_{1} \\
\varphi_{2}
\end{array}\right) \rightarrow\left(\begin{array}{c}
e^{-f} \varphi_{1} \\
e^{-\bar{f}} \varphi_{2}
\end{array}\right),
$$

где $f$ - произвольная функция. Но отображения

$$
G_{\psi}=\left(\psi_{1}: \bar{\psi}_{2}\right), \quad G_{\varphi}=\left(\varphi_{1}: \bar{\varphi}_{2}\right)
$$

в $\mathbb{C} P^{1}$ корректно определены и задают разбиение гауссова отображения

$$
G=\left(G_{\psi}, G_{\varphi}\right): \Sigma \rightarrow \widetilde{G}_{4,2}=\mathbb{C} P^{1} \times \mathbb{C} P^{1} .
$$

Мы имеем следующие формулы для погружения поверхности:

$$
x^{k}=x^{k}(0)+\int\left(x_{z}^{k} d z+\bar{x}_{z}^{k} d \bar{z}\right), \quad k=1,2,3,4,
$$

где

$$
\begin{aligned}
x_{z}^{1} & =\frac{i}{2}\left(\bar{\varphi}_{2} \bar{\psi}_{2}+\varphi_{1} \psi_{1}\right), & x_{z}^{2} & =\frac{1}{2}\left(\bar{\varphi}_{2} \bar{\psi}_{2}-\varphi_{1} \psi_{1}\right), \\
x_{z}^{3} & =\frac{1}{2}\left(\bar{\varphi}_{2} \psi_{1}+\varphi_{1} \bar{\psi}_{2}\right), & x_{z}^{4} & =\frac{i}{2}\left(\bar{\varphi}_{2} \psi_{1}-\varphi_{1} \bar{\psi}_{2}\right) .
\end{aligned}
$$

Конечно, как и в трехмерном случае, эти формулы задают поверхность тогда и только тогда, когда подынтегральные выражения - замкнутые формы или, что эквивалентно,

$$
\operatorname{Im} x_{z \bar{z}}^{k}=0, \quad k=1,2,3,4 .
$$

Это условие переписывается как

$$
\left(\bar{\varphi}_{2} \psi_{1}\right)_{\bar{z}}=\left(\bar{\varphi}_{1} \psi_{2}\right)_{z}, \quad\left(\bar{\varphi}_{2} \bar{\psi}_{2}\right)_{\bar{z}}=-\left(\bar{\varphi}_{1} \bar{\psi}_{1}\right)_{z}
$$

Для $\varphi$ и $\psi$ общего положения это условие не переписывается в терминах уравнений Дирака.

Но имеет место следующая теорема.

Теорема 4. Пусть $r: W \rightarrow \mathbb{R}^{4}$ - погруженная поверхность с конформным параметром z и $G_{\psi}=\left(e^{i \theta} \cos \eta: \sin \eta\right)$ - одна из компонент гауссова отображения.

Существует другой представитель $\psi$ отображения $G_{\psi}=\left(\psi_{1}: \bar{\psi}_{2}\right)$ такой, что он удовлетворяет уравнению Дирака

$$
\mathscr{D} \psi=0, \quad \mathscr{D}=\left(\begin{array}{cc}
0 & \partial \\
-\bar{\partial} & 0
\end{array}\right)+\left(\begin{array}{cc}
U & 0 \\
0 & \bar{U}
\end{array}\right)
$$


с некоторым потенииалом $U$.

Вектор-функиия $\psi=\left(e^{g+i \theta} \cos \eta, e^{\bar{g}} \sin \eta\right)$ определяется из уравнения

$$
g_{\bar{z}}=-i \theta_{\bar{z}} \cos ^{2} \eta,
$$

чве решение g определено с точностью до прибавления произвольной голоморфной бункции $h$, и соответствующий потенциал $U$ задается формулой

$$
U=-e^{\bar{g}-g-i \theta}\left(i \theta_{z} \sin \eta \cos \eta+\eta_{z}\right)
$$

с точностъю до умножения на $e^{\bar{h}-h}$.

Если задана вектор-функция $\psi$, то вектор-функция $\varphi$, представляющая другую компоненту $G_{\varphi}$ отображения Гаусса, удовлетворяет уравнению

$$
\mathscr{D}^{\vee} \varphi=0, \quad \mathscr{D}^{\vee}=\left(\begin{array}{cc}
0 & \partial \\
-\bar{\partial} & 0
\end{array}\right)+\left(\begin{array}{cc}
\bar{U} & 0 \\
0 & U
\end{array}\right) .
$$

Различные подбемы в $\mathbb{C}^{2} \times \mathbb{C}^{2}$ отображения Гаусса $G: \Sigma \rightarrow \mathbb{C} P^{1} \times \mathbb{C} P^{1}$ связаны калибровочными преобразованиями

$$
\left(\begin{array}{l}
\psi_{1} \\
\psi_{2}
\end{array}\right) \rightarrow\left(\begin{array}{c}
e^{h} \psi_{1} \\
e^{\bar{h}} \psi_{2}
\end{array}\right), \quad\left(\begin{array}{l}
\varphi_{1} \\
\varphi_{2}
\end{array}\right) \rightarrow\left(\begin{array}{c}
e^{-h} \varphi_{1} \\
e^{-\bar{h}} \varphi_{2}
\end{array}\right), \quad U \rightarrow \exp (\bar{h}-h) U,
$$

где $h$ - произвольная голоморфная функция на $W$.

СЛЕДСТвИЕ 1. Каждая ориентированная поверхность в $\mathbb{R}^{4}$ задается формулами (17) и (18), где вектор-функиии $\psi$ и ч удовлетворяют уравнениям типа Дирака (20) и (22):

$$
\mathscr{D} \psi=\mathscr{D}^{\vee} \varphi=0 .
$$

Индуиированная метрика имеет вид

$$
e^{2 \alpha} d z d \bar{z}=\left(\left|\psi_{1}\right|^{2}+\left|\psi_{2}\right|^{2}\right)\left(\left|\varphi_{1}\right|^{2}+\left|\varphi_{2}\right|^{2}\right) d z d \bar{z}
$$

и норма вектора средней кривизны $\mathbf{H}=2 x_{z \bar{z}} / e^{2 \alpha}$ удовлетворяет равенству

$$
|U|=\frac{|\mathbf{H}| e^{\alpha}}{2} .
$$

Рассмотрим диагональное вложение

$$
\widetilde{G}_{3,2}=\mathbb{C} P^{1} \rightarrow \widetilde{G}_{4,2}=\mathbb{C} P^{1} \times \mathbb{C} P^{1} .
$$

Если $\varphi$ и $\psi$ порождают поверхность и лежат на диагонали: $\varphi= \pm \psi$, то $x^{4}=0$ и мы получаем представление Вейерштрасса поверхности в $\mathbb{R}^{3}$.

Формулы (17) и (18) появились в [9] для построения поверхностей. Это следствие показывает, что они имеют общий характер, хотя это должно следовать из [8], где такое представление впервые было указано на кватернионном языке.

Мы укажем два особых свойства представления поверхностей в $\mathbb{R}^{4}$, которые не обсуждались в предыдущих статьях:

а) для заданной поверхности представление неединственно и различные представления связаны нетривиальными калибровочными преобразованиями; 
б) представление Вейерштрасса некоторой области не всегда продолжается до представления всей поверхности и для того, чтобы получить представление всей поверхности, надо решить на ней $\bar{\partial}$-задачу (21).

Действительно, возьмем $\psi$ и $\varphi$, порождающие поверхность $\Sigma$, область $W \subset \Sigma$ и голоморфную функцию $f$ на $W$, которая не продолжается аналитически вне $W$. Затем построим с помощью (16) по $\psi, \varphi$ и $f$ иное представление $W$, которое не продолжается вне $W$.

ПРИМЕР. ЛАГРАНЖЕВЫ ПОВЕРХНОСТИ В $\mathbb{R}^{4}$. МЫ изложим представление Вейерштрасса для лагранжевых поверхностей в $\mathbb{R}^{4}$, полученное Эла и Ромоном [33]. Редукция формул (18) к формулам из [33] была указана Эла [34].

Возьмем следующую симплектическую форму на $\mathbb{R}^{4}$ :

$$
\omega=d x^{1} \wedge d x^{2}+d x^{3} \wedge d x^{4} .
$$

Напомним, что $n$-мерное подмногообразие $\Sigma$ в $2 n$-мерном симплектическом многообразии $M^{2 n}$ с симплектической формой $\omega$ называется лагранжевым, если ограничение $\omega$ на $\Sigma$ равно нулю:

$$
\left.\omega\right|_{\Sigma}=0
$$

Это означает, что в каждой точке $x \in \Sigma$ ограничение формы $\omega$ на касательное пространство $T_{x} \Sigma$ равно нулю, т.е. $T_{x} \Sigma$-лагранжева $n$-мерная плоскость в $\mathbb{R}^{2 n}$.

Условие, что двумерная плоскость лагранжева в $\mathbb{R}^{4}$, записывается как

$$
\operatorname{Im}\left(y_{1} \bar{y}_{2}+y_{3} \bar{y}_{4}\right)=0
$$

или

$$
\left|y_{1}^{\prime}\right|^{2}-\left|y_{2}^{\prime}\right|^{2}-\left|y_{3}^{\prime}\right|^{2}+\left|y_{4}^{\prime}\right|^{2}=0 .
$$

В терминах $a_{1}, a_{2}, b_{1}$ и $b_{2}$ это условие принимает вид

$$
\left|b_{1}\right|^{2}=\left|b_{2}\right|^{2}
$$

Следовательно, грассманиан лагранжевых двумерных плоскостей в $\mathbb{R}^{4}$ является произведением многообразий

$$
G_{4,2}^{\mathrm{Lag}}=\mathbb{C} P^{1} \times S^{1}
$$

где $\left(a_{1}: a_{2}\right)$ параметризует $\mathbb{C} P^{1}$ и окружность $S^{1}$ параметризована величиной

$$
\beta=\frac{1}{i} \log \frac{b_{1}}{b_{2}} \bmod 2 \pi,
$$

которая называется лагранжевым углом.

Мы заключаем, что поверхность лагранжева тогда и только тогда, когда

$$
\left|\psi_{1}\right|=\left|\psi_{2}\right|
$$

в ее представлении Вейерштрасса. Положим

$$
s=\left(\frac{e^{i \beta}}{\sqrt{2}}, \frac{1}{\sqrt{2}}\right), \quad\left(s_{1}: s_{2}\right)=\left(\psi_{1}: \bar{\psi}_{2}\right) \in \mathbb{C} P^{1}
$$


и применим теорему 4. Мы получим следующие формулы:

$$
g=-\frac{i \beta}{2}, \quad U=-\frac{1}{2} \beta_{z}, \quad \psi_{1}=\psi_{2}=\frac{1}{\sqrt{2}} e^{i \beta / 2} .
$$

Для каждого решения $\varphi$ уравнения $\mathscr{D}^{\vee} \varphi=0$ мы получаем лагранжеву поверхность, заданную вектор-функциями $\psi$ и $\varphi$ по формулам (18). Более того, все лагранжевы поверхности представляются в таком виде.

Пусть

$$
f: \Sigma \rightarrow \mathbb{R}^{4}
$$

- погружение ориентированной замкнутой поверхности в $\mathbb{R}^{4}$. По теореме 4 эта поверхность локально определяется формулами (17) и (18). Глобализация этого представления аналогична случаю поверхностей в $\mathbb{R}^{3}$ и на кватернионном языке была описана в [8], [12], но для получения его необходимо решить $\bar{\partial}-$ задачу на поверхности [35]:

ПреДЛОЖЕНИЕ 3. Для представления Вейерштрасса погруженной ориентированной замкнутой поверхности $\Sigma$ в $\mathbb{R}^{4}$ соответствующие функции $\psi$ и ч являются сечениями $\mathbb{C}^{2}$-расслоений $E$ и $E^{\vee}$ над $\Sigma$, которые имеют следующий вид:

1) $E$ и $E^{\vee}$ распадаются в суммы попарно сопряженных линейных расслоениนั

$$
E=E_{0} \oplus \bar{E}_{0}, \quad E^{\vee}=E_{0}^{\vee} \oplus \bar{E}_{0}^{\vee}
$$

так, что $\psi_{1} u \bar{\psi}_{2}-$ сечения $E_{0}, a \varphi_{1} u \bar{\varphi}_{2}-$ сечения $E_{0}^{\vee}$;

2) спаривание сечений $E_{0}$ и $E_{0}^{\vee}$ дает $(1,0)$-форму на $\Sigma$ : если

$$
\alpha \in \Gamma\left(E_{0}\right), \quad \beta \in \Gamma\left(E_{0}^{\vee}\right),
$$

mo

$$
\alpha \beta d z
$$

- корректно определенная 1-форма на $\Sigma$;

3) из уравнения Дирака $\mathscr{D} \psi=0$ следует, что $U$ - это сечение того же самого линейного расслоения $E_{U}$, что и

$$
\frac{\partial \gamma}{\alpha} \in \Gamma\left(E_{U}\right) \quad \text { при } \quad \alpha \in \Gamma\left(E_{0}\right), \quad \gamma \in \Gamma\left(\bar{E}_{0}\right)
$$

u $U \bar{U} d z \wedge d \bar{z}-$ корректно определенная $(1,1)$-форма на $\Sigma$, чей интеграл по поверхности равен

$$
\int_{\Sigma} U \bar{U} d z \wedge d \bar{z}=-\frac{i}{2} \mathscr{W}(\Sigma)
$$

где $\mathscr{W}(\Sigma)=\int_{\Sigma}|\mathbf{H}|^{2} d \mu-$ функционал Уиллмора.

Калибровочное преобразование (23) показывает, что в отличие от трехмерного случая $\psi$ не обязательно являются сечениями спинорных расслоений.

Для торов из теоремы 4 мы выводим следующий результат. 
Теорема 5 [35]. Пусть $\Sigma-$ тор в $\mathbb{R}^{4}$, который конформно эквивалентен $\mathbb{C} / \Lambda$ и z-конформный параметр на нем.

Тогда существуют такие вектор-функиии $\psi$ и ч и функция $U$ на $\mathbb{C}$, что

1) $\psi$ и ч задают представление Вейерштрасса поверхности $\Sigma$;

2) потенциал $U$ этого представления $\Lambda$-периодичен;

3) функиии $\psi, \varphi$ и U, удовлетворяющие 1) и 2), определяются с точностью до калибровочных преобразований

$$
\left(\begin{array}{l}
\psi_{1} \\
\psi_{2}
\end{array}\right) \rightarrow\left(\begin{array}{c}
e^{h} \psi_{1} \\
e^{\bar{h}} \psi_{2}
\end{array}\right), \quad\left(\begin{array}{l}
\varphi_{1} \\
\varphi_{2}
\end{array}\right) \rightarrow\left(\begin{array}{c}
e^{-h} \varphi_{1} \\
e^{-\bar{h}} \varphi_{2}
\end{array}\right), \quad U \rightarrow e^{\bar{h}-h} U
$$

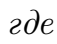

$$
h(z)=a+b z, \quad \operatorname{Im}(b \gamma) \in \pi \mathbb{Z} \quad \text { для всех } \gamma \in \Lambda .
$$

Как и в случае поверхностей в $\mathbb{R}^{3}$, в общем случае вектор-функции $\psi$ и $\varphi$ определяют погружение в $\mathbb{R}^{4}$ универсального накрытия $\widetilde{\Sigma}$ поверхности $\Sigma$.

ПредлоЖениЕ 4. Погружение $\widetilde{\Sigma}$ факторизуется в погружение $\Sigma$, если и только если

$$
\int_{\Sigma} \bar{\psi}_{1} \bar{\varphi}_{1} d \bar{z} \wedge \omega=\int_{\Sigma} \bar{\psi}_{1} \varphi_{2} d \bar{z} \wedge \omega=\int_{\Sigma} \psi_{2} \bar{\varphi}_{1} d \bar{z} \wedge \omega=\int_{\Sigma} \psi_{2} \varphi_{2} d \bar{z} \wedge \omega=0
$$

для каждого голоморфного дифференииала $\omega$ на $\Sigma$.

При $\psi_{1}= \pm \varphi_{1}, \psi_{2}= \pm \varphi_{2}$ формула (25) переходит в (7).

\section{§ 3. Интегрируемые деформации поверхностей}

3.1. Модифицированное уравнение Веселова-Новикова. Иерархия модифицированных уравнений Веселова-Новикова (мBН) была введена Богдановым [36], [37], и уравнения этой иерархии имеют вид “ $L, A, B$ ”-троек

$$
\frac{\partial L}{\partial t_{n}}=\left[L, A_{n}\right]-B_{n} L
$$

где $L=\mathscr{D}$ - оператор Дирака

$$
L=\left(\begin{array}{cc}
0 & \partial \\
-\bar{\partial} & 0
\end{array}\right)+\left(\begin{array}{cc}
U & 0 \\
0 & U
\end{array}\right)
$$

и $A_{n}, B_{n}$ - матричные дифференциальные операторы, при этом старший член оператора $A_{n}$ имеет следующий вид:

$$
A_{n}=\left(\begin{array}{cc}
\partial^{2 n+1}+\bar{\partial}^{2 n+1} & 0 \\
0 & \partial^{2 n+1}+\bar{\partial}^{2 n+1}
\end{array}\right)+\cdots
$$

В отличие от “ $L, A$ "-пар “ $L, A, B$ "-тройка сохраняет только нулевой уровень энергии оператора $L$, деформируя соответствующие собственные функции. Действительно, мы имеем

$$
\frac{\partial L \psi}{\partial t}=L_{t} \psi+L \psi_{t}=L\left[\left(A+\partial_{t}\right) \psi\right]-(A+B)[L \psi] .
$$


Следовательно, если $\psi$ удовлетворяет уравнению

$$
\frac{\partial \psi}{\partial t}+A \psi=0
$$

и $L \psi_{0}=0$ для начальных данных $\psi_{0}=\left.\psi\right|_{t=t_{0}}$ этого эволюционного уравнения, то

$$
L \psi=0
$$

для всех $t \geqslant t_{0}$.

При $n=1$ мы имеем исходное уравнение мВН

$$
U_{t}=\left(U_{z z z}+3 U_{z} V+\frac{3}{2} U V_{z}\right)+\left(U_{\bar{z} \bar{z} \bar{z}}+3 U_{\bar{z}} \bar{V}+\frac{3}{2} U \bar{V}_{\bar{z}}\right),
$$

где

$$
V_{\bar{z}}=\left(U^{2}\right)_{z}
$$

Мы видим, что если начальные данные задачи Коши $\left.U\right|_{t=0}-$ вещественнозначная функция, то решение тоже вещественнозначно. В случае, когда функция $\left.U\right|_{t=0}$ зависит только от переменной $x$, мы имеем $U=U(x, t)$ и уравнение мВН сводится к модифицированному уравнению Кортевега-де Фриза

$$
U_{t}=\frac{1}{4} U_{x x x}+6 U_{x} U^{2}
$$

(здесь $V=U^{2}$ ).

Эта редукция объясняет название уравнения, поскольку Веселов и Новиков ввели в [38], [39] иерархию $(2+1)$-мерных солитонных уравнений, которые имеют вид “ $L, A, B$ "-троек для скалярного оператора $L=\partial \bar{\partial}+U$ (двумерного потенциального оператора Шрёдингера) и в $(1+1)$-пределе переходят в уравнение Кортевега-де Фриза. Оригинальное уравнение Веселова-Новикова имеет вид

$$
U_{t}=U_{z z z}+U_{\bar{z} \bar{z} \bar{z}}+(V U)_{z}+(\bar{V} U)_{\bar{z}}, \quad V_{\bar{z}}=3 U_{z}
$$

и его вывод был позднее модифицирован Богдановым для вывода уравнения $\mathrm{MBH}$.

Из формул (2) и (3) представления Вейерштрасса следует, что именно нулевой уровень энергии оператора Дирака отвечает поверхностям в $\mathbb{R}^{3}$. Это приводит к следующей теореме.

Теорема 6 [2]. Пусть $U(z, \bar{z}, t)$ - вещественнозначное решение уравнения мBН (27). Пусть $\Sigma$ - поверхность, построенная с помощъю представления Вейерштрасса (2) и (3) по вектор-функции $\psi_{0}$ такой, что $\psi_{0}$ удовлетворяет уравнению Дирака $\mathscr{D} \psi_{0}=0$ с потенциалом $U=U(z, \bar{z}, 0)$. Пусть $\psi(z, \bar{z}, t)-$ решение уравнения (26) с начальными данными $\left.\psi\right|_{t=0}=\psi_{0}$.

Тогда поверхности $\Sigma(t)$, построенные по $\psi(z, \bar{z}, t)$ с помощъю представления Вейерштрасса, задают солитонную деформацию поверхности $\Sigma$.

Деформация, данная этой теоремой, называется мBН-деформацией поверхности.

Конечно, эта теорема верна для всех уравнений иерархии мВН. Рекурсионная формула для них до сих пор неизвестна, и до последнего времени следующие уравнения не были выписаны явно, за исключением случая $n=2$ [1]. Конечнозонные решения уравнений мВН построены в [40] (см. также [41]). 
В [1] было установлено, что эта деформация имеет глобальный характер для торов и сохраняет функционал Уиллмора.

Теорема 7 [1]. Дебормаиия мBН переводит торы в торы и сохраняет их конформные классы и значения функционала Уиллмора.

Доказательство этой теоремы состоит в следующем. Для того чтобы корректно определить эту деформацию, надо разрешить условие (28), и для торов это можно сделать, как показано в [1]. Мы должны взять решение $V$ уравнения (28), нормированное условием

$$
\int_{\Sigma} V d z \wedge d \bar{z}=0
$$

Форма $\left(U^{2}\right)_{t} d z \wedge d \bar{z}$ является точной формой на торе $\Sigma$ :

$$
U U_{t}=\left(U U_{z z}-\frac{U_{z}^{2}}{2}+\frac{3}{2} U^{2} V\right)_{z}+\left(U U_{\bar{z} \bar{z}}-\frac{U_{\bar{z}}^{2}}{2}+\frac{3}{2} U^{2} \bar{V}\right)_{\bar{z}}
$$

и, следовательно, функционал Уиллмора сохраняется:

$$
\frac{d}{d t} \int_{\Sigma} U^{2} d z \wedge d \bar{z}=\int_{\Sigma}\left(U^{2}\right)_{t} d z \wedge d \bar{z}=0
$$

Плоская структура на торе позволяет идентифицировать дифференциалы с периодическими функциями. $\mathrm{K}$ примеру, формально $U^{2} d z d \bar{z}-$ это $(1,1)$ дифференциал, а $V d z^{2}-$ квадратичный дифференциал. Это невозможно для поверхностей большего рода и тем самым препятствует глобальному определению мВН-деформаций таких поверхностей.

Некоторая попытка переопределить солитонные деформации в полностью геометрических терминах была предпринята в [42]. В итоге не удалось избежать введения конформного параметра на поверхности, но удалось вскрыть интересные геометрические свойства деформаций.

После работ [2], [1] в рамках аффинной геометрии и сферической геометрии Ли были введены и изучены некоторые другие солитонные деформации с геометрическими законами сохранения [43]-[45].

3.2. Модифицированное уравнение Кортевега-де Фриза. В случае, когда потенциал $U$ зависит только от $x=\operatorname{Re} z$, уравнение Дирака $\mathscr{D} \psi=0$ для функций вида

$$
\psi(z, \bar{z})=\varphi(x) \exp \left(\frac{i y}{2}\right)
$$

сводится к задаче Захарова-Шабата

$$
L \varphi=0, \quad L=\left[\left(\begin{array}{cc}
0 & 1 \\
-1 & 0
\end{array}\right) \frac{d}{d x}+\left(\begin{array}{cc}
q & -i k \\
-i k & q
\end{array}\right)\right], \quad q=2 U,
$$

при $k=i / 2$.

Заметим, что для поверхностей вращения функция $\psi$ принимает вид (30) по отношению к некоторой конформной координате $z=x+i y$, где $y$ - угол вращения. Но существует много других поверхностей с внутренней $S^{1}$-симметрией, для которых потенциал $U$ зависит только от $x$. Функция $\varphi$ периодична для торов вращения и быстро убывает для сфер вращения [6]. 
Оператор $L$ ассоциирован с солитонной иерархией модифицированных уравнений Кортевега-де Фриза, которые имеют представление в виде “ $L, A$ "-пары

$$
\frac{d L}{d t}=\left[L, A_{n}\right]
$$

Простейшие из них -

$$
\begin{gathered}
q_{t}=q_{x x x}+\frac{3}{2} q^{2} q_{x}, \quad n=1, \\
q_{t}=q_{x x x x x}+\frac{5}{2} q^{2} q_{x x x}+10 q q_{x} q_{x x}+\frac{5}{2} q_{x}^{3}+\frac{15}{8} q^{4} q_{x}, \quad n=2 .
\end{gathered}
$$

Первое из них совпадает с редукцией (29) уравнения мВН после подстановки $q \rightarrow 4 U$ и замены масштаба временной переменной $t \rightarrow 4 t$. В действительности, иерархия мКдФ является редукцией иерархии мВН при $U=U(x)$.

Мы видим, что в случае уравнений мКдФ нет условий связи вида (28) и можно легко определять мКдФ-деформации поверхностей вращения. Более того, в этом случае существует рекурсионная формула для высших уравнений:

$$
\frac{\partial q}{\partial t_{n}}=D^{n} q_{x}, \quad D=\partial_{x}^{2}+q^{2}+q_{x} \partial_{x}^{-1} q .
$$

Введем интегралы Крускала-Миуры. Их плотности $R_{k}$ определяются следующей рекурсионной процедурой:

$$
R_{1}=\frac{i q_{x}}{2}-\frac{q^{2}}{4}, \quad R_{n+1}=-R_{n x}-\sum_{k=1}^{n-1} R_{k} R_{n-k}
$$

Можно показать, что $R_{2 n}$ - полные производные и только интегралы

$$
H_{k}=\int R_{2 k-1} d x
$$

не равны нулю тождественно.

Теорема 8 [46]. Для каждого $n \geqslant 1$ n-е уравнение иерархии мКдФ преобразует (как редукиия мВН-дебормации) торы вращения в торы вращения с сохранением конформных типов и значений функиионалов $H_{k}, k \geqslant 1$.

Доказательство аналогичной теоремы для сфер вращения (они изучены в [6]) фактически то же самое, что и для торов.

Заметим, что сохранение торов не является тривиальным утверждением. Данный оператор $L$ входит также в “ $L, A$ "-пару для уравнения sin-Гордон, которое тем самым тоже порождает деформации поверхностей вращения. Однако эта деформация раскрывает торы в цилиндры.

Мы видим, что

$$
H_{1}=-\frac{1}{4} \int q^{2} d x=-4 \int U^{2} d x=-\frac{2}{\pi} \int U^{2} d x \wedge d y
$$

и, следовательно, первый интеграл Крускала-Миуры пропорционален функционалу Уиллмора. Следующие интегралы имеют вид

$$
H_{2}=\frac{1}{16} \int\left(q^{2}-4 q_{x}^{2}\right) d x, \quad H_{3}=\frac{1}{32} \int\left(q^{6}-20 q^{2} q_{x}^{2}+8 q_{x} x^{2}\right) d x .
$$

Интересно было бы понять, 
каков геометрический смысл функционалов $H_{k}$ и каковы экстремали этих функционалов среди компактных поверхностей вращения?

мКдФ-деформации поверхностей вращения задают деформации кривых, порождающих поверхности при вращении, в верхней полуплоскости. Геометрия таких деформаций и взаимосвязь рекурсионных соотношений и геометрии кривых изучались в [47], [48].

3.3. Уравнение Дэви-Стюартсона. Уравнения мВН сами являются редукциями при $U=-p=\bar{q}$ уравнений Дэви-Стюартсона (ДС), заданных " $L, A, B$ "-тройками с

$$
L=\left(\begin{array}{cc}
0 & \partial \\
-\bar{\partial} & 0
\end{array}\right)+\left(\begin{array}{cc}
-p & 0 \\
0 & q
\end{array}\right) .
$$

В действительности эта редукция уравнений ДС дает больше уравнений, имеющих вид

$$
U_{t}=i\left(\partial^{2 n} U+\bar{\partial}^{2 n} U\right)+\cdots
$$

или

$$
U_{t}=\partial^{2 n+1} U+\bar{\partial}^{2 n+1} U+\cdots
$$

при $n \geqslant 1$. Уравнения из первого семейства не сохраняют условие вещественности $U=\bar{U}$, а второе семейство при $U=\bar{U}$ сводится к иерархии мВН.

Первые два из этих уравнений - это уравнение ДС 2

$$
U_{t}=i\left(U_{z z}+U_{\bar{z} \bar{z}}+2(V+\bar{V}) U\right)
$$

где

$$
V_{\bar{z}}=\partial\left(|U|^{2}\right),
$$

и уравнение ДС 3 (которое иногда называется уравнением Дэви-Стюартсона I):

$$
U_{t}=U_{z z z}+U_{\bar{z} \bar{z} \bar{z}}+3\left(V U_{z}+\bar{V} U_{\bar{z}}\right)+3\left(W+W^{\prime}\right) U
$$

где

$$
V_{\bar{z}}=\left(|U|^{2}\right)_{z}, \quad W_{\bar{z}}=\left(\bar{U} U_{z}\right)_{z}, \quad W_{z}^{\prime}=\left(\bar{U} U_{\bar{z}}\right)_{\bar{z}} .
$$

Уравнения ДС задают солитонные деформации поверхностей в $\mathbb{R}^{4}$. Как и для поверхностей в $\mathbb{R}^{3}$, эти деформации были введены Конопельченко, доказавшим в [9] соответствующий аналог теоремы 6 .

Однако в этом случае есть две специфических проблемы.

1) Как мы уже отмечали в п. 2.5, представление Вейерштрасса поверхности в $\mathbb{R}^{4}$ неединственно. Являются ли деформации ДС геометрически различными для разных представлений?

2) Условия связи для уравнений ДС более сложные и как разрешать условия (32) и (34), чтобы получить глобальные деформации замкнутых поверхностей?

Эти задачи мы рассмотрели в [35].

Ответ на первый вопрос демонстрирует большое различие с деформациями $\mathrm{MBH}$ :

деформации ДС корректно определены только для поверхностей с заданными потенциалами $U$ их представлений Вейерштрасса и для различных выборов потенциала эти деформации геометрически различны. 
Было бы интересно понять геометрический смысл этих различных деформаций одной и той же поверхности.

Ответ на второй вопрос дается следующим аналогом теоремы 7.

ТЕОРема 9. 1) Для функиии $V$, однозначно определенной уравнением (32) и нормировкой $\int V d z \wedge d \bar{z}=0$, уравнение Д $C_{2}$ задает деформачии торов в торь с сохранением их конформных классов и значений функиионала Уиллмора.

2) $\Pi p u$

$$
V_{\bar{z}}=\left(|U|^{2}\right)_{z}, \quad \int V d z \wedge d \bar{z}=0, \quad W=\partial \bar{\partial}^{-1}\left(\bar{u} u_{z}\right), \quad W^{\prime}=\bar{\partial} \partial^{-1}\left(\bar{u} u_{\bar{z}}\right)
$$

уравнение ДС $C_{3}$ задает деформацию торов в торь, которая сохраняет их конформные классы и функиионал Уиллмора.

Поверхность деформируется посредством деформаций вектор-функций $\psi$ и $\varphi$, и эти деформации связаны с оператором $A$ из “ $L, A, B$ "-тройки. Существует значительно больше дополнительных потенциалов, входящих в $A$ и уравнения ДС, как объяснено в [9]. Мы не объясняем здесь редукции в формуле для $A$, которые необходимы для сохранения замкнутости поверхностей при деформациях. Мы только заметим, что формула (34) определяет периодические потенциалы $W$ и $W^{\prime}$ с точностью до постоянных и формула (35) нормирует эти постоянные. Эта нормировка необходима для сохранения функционала Уиллмора. Разрешение всех эти условий изложено в статье [35], к которой мы отсылаем за всеми подробностями.

\section{§ 4. Спектральные кривые}

4.1. Некоторые факты из функционального анализа. Для заданной области $\Omega \subset \mathbb{R}^{n}$ обозначим через $L_{p}(\Omega)$ и $W_{p}^{k}$ пространства Соболева, которые являются замыканиями пространства финитных замкнутых функций на $\Omega$ относительно норм

$$
\|f\|_{p}=\int_{\Omega}|f(x)|^{p} d x_{1} \cdots d x_{n}
$$

И

$$
\|f\|_{k, p}=\sum_{0 \leqslant l_{1}+\cdots+l_{n}=l \leqslant k} \int_{\Omega}\left|\frac{\partial^{l} f}{\partial^{l_{1}} x_{1} \cdots \partial^{l_{n}} x_{n}}\right|^{p} d x_{1} \cdots d x_{n} .
$$

Для тора $T^{n}=\mathbb{R}^{n} / \Lambda$ мы обозначим через $L_{p}\left(T^{n}\right)$ и $W_{p}^{k}\left(T^{n}\right)$ аналогичные пространства Соболева, образованные $\Lambda$-периодическими функциями. При этом интегралы в определениях норм взяты по компактной фундаментальной области группы сдвигов $\Lambda$.

ПредЛОЖЕНИЕ 5. Пусть $\Omega$ - компактная замкнутая область в $\mathbb{R}^{n}$ или тор. Тогда

а) (Реллих) существует естественное непрерьвное вложение $W_{p}^{k}(\Omega) \rightarrow$ $L_{p}(\Omega)$, которое компактно при $k>0$;

б) (Гёльдер) умножение на $u \in L_{p}$ - это ограниченный оператор из $L_{q} 8$ $L_{r}$ u при этом $\|u v\|_{r} \leqslant\|u\|_{p}\|v\|_{q}, 1 / p+1 / q=1 / r$

в) (Соболев) существует непрерывное вложение $W_{p}^{1}(\Omega) \rightarrow L_{q}(\Omega), q \leqslant$ $n p /(n-p)$, чъя норма называется постоянной Соболева; 
г) (Кондрашов) при $q<n p /(n-p)$ вложение Соболева компактно.

Мы будем обозначать пространства двухкомпонентных векторных функций на торе $M=\mathbb{R}^{2} / \Lambda$ через

$$
L_{p}=L_{p}(M) \times L_{p}(M), \quad W_{p}^{k}=W_{p}^{k}(M) \times W_{p}^{k}(M)
$$

в отличие от пространств скалярных функций $L_{2}(M)$ и $W_{p}^{1}(M)$.

Пусть $H$ - гильбертово пространство. Оператор $A: H \rightarrow H-$ компактный, если для единичного шара $B=\{|x|<1: x \in H\}$ замыкание его образа $A(B)$ компактно. Спектр $\operatorname{Spec} A$ компактного оператора $A$ ограничен и может иметь предельную точку только в нуле.

Для заданного гильбертова пространства $H$ и оператора $A$ (не обязательно ограниченного) обозначим через $R(\lambda)$ резольвенту оператора $A$. Это - операторный пучок

$$
R(\lambda)=(A-\lambda)^{-1},
$$

имеющий полюсы в точках из $\operatorname{Spec} A$ и голоморфный по $\lambda$ вне $\operatorname{Spec} A$.

Тождество Гильберта утверждает, что

$$
R(\mu) R(\lambda)=\frac{1}{\mu-\lambda}(R(\lambda)-R(\mu))
$$

или, в других обозначениях,

$$
\frac{1}{A-\mu} \frac{1}{A-\lambda}=\frac{1}{\mu-\lambda}\left(\frac{1}{A-\lambda}-\frac{1}{A-\mu}\right) .
$$

Если в некоторой области из $\mathbb{C}$ задана резольвента, то она продолжается на всю плоскость $\mathbb{C}$ с помощью следующего следствия из тождества Гильберта:

$$
R(\mu)=R(\lambda)((\mu-\lambda) R(\lambda)+1)^{-1}
$$

(заметим, что $R(\lambda) R(\mu)=R(\mu) R(\lambda)$ ).

ПРЕДЛОЖЕНИЕ 6. Если оператор $R(\lambda)$ компактен при $\lambda=\lambda_{0}$ и голоморфен по $\lambda$ около $\lambda_{0}$, то

1) резольвента $R(\mu)$ компактна для любого $\mu \in \mathbb{C} \backslash$ Spec $A$ и имеет полюсъ только в точках из $\operatorname{Spec} A$;

2) $R(\lambda)$ голоморфна в $\mathbb{C} \backslash \operatorname{Spec} A$.

4.2. Спектральная кривая оператора Дирака с ограниченными потенциалами. В этом пункте мы изложим схему доказательства существования спектральной кривой для дифференциального оператора с периодическими коэффициентами, которую мы использовали в [3] для операторов Дирака с ограниченными потенциалами. Этот случай покрывает все операторы Дирака, отвечающие торам в $\mathbb{R}^{3}$.

Пусть

$$
\mathscr{D}=\left(\begin{array}{cc}
0 & \partial \\
-\bar{\partial} & 0
\end{array}\right)+\left(\begin{array}{cc}
U & 0 \\
0 & V
\end{array}\right)=\mathscr{D}_{0}+\left(\begin{array}{cc}
U & 0 \\
0 & V
\end{array}\right) .
$$

Здесь мы обозначаем через $\mathscr{D}_{0}$ свободный оператор Дирака:

$$
\mathscr{D}_{0}=\left(\begin{array}{cc}
0 & \partial \\
-\bar{\partial} & 0
\end{array}\right) \text {. }
$$


Собственная функция Флоке $\psi$ оператора $\mathscr{D}$ с собственным значением (или энергией) $E$ - это формальное решение уравнения

$$
\mathscr{D} \psi=E \psi
$$

отвечающее следующим условиям периодичности:

$$
\psi\left(z+\gamma_{j}, \bar{z}+\bar{\gamma}_{j}\right)=e^{2 \pi i\left(k, \gamma_{j}\right)} \psi(z, \bar{z})=\mu\left(\gamma_{j}\right) \psi(z, \bar{z}), \quad j=1,2,
$$

где

$$
\left(k, \gamma_{j}\right)=k_{1} \gamma_{j}^{1}+k_{2} \gamma_{j}^{2}, \quad \gamma_{j}=\gamma_{j}^{1}+i \gamma_{j}^{2} \in \mathbb{C}=\mathbb{R}^{2}, \quad k=\left(k_{1}, k_{2}\right) .
$$

Величины $k_{1}, k_{2}$ называются квазиимпульсами функции $\psi$ и $\left(\mu_{1}, \mu_{2}\right)=\left(\mu\left(\gamma_{1}\right)\right.$, $\left.\mu\left(\gamma_{2}\right)\right)$ называются мультипликаторами функции $\psi$.

Представим собственную функцию Флоке $\psi$ в виде произведения

$$
\psi(z, \bar{z})=e^{2 \pi i\left(k_{1} x+k_{2} y\right)} \varphi(z, \bar{z}), \quad z=x+i y, \quad x, y \in \mathbb{R},
$$

где функция $\varphi(z, \bar{z})-\Lambda$-периодическая. Уравнение $\mathscr{D} \psi=E \psi$ принимает вид

$$
\left[\left(\begin{array}{cc}
0 & \partial \\
-\bar{\partial} & 0
\end{array}\right)+\left(\begin{array}{cc}
U & \pi i\left(k_{1}-i k_{2}\right) \\
-\pi i\left(k_{1}+i k_{2}\right) & V
\end{array}\right)\right]\left(\begin{array}{l}
\varphi_{1} \\
\varphi_{2}
\end{array}\right)=E\left(\begin{array}{l}
\varphi_{1} \\
\varphi_{2}
\end{array}\right) .
$$

Мы имеем операторный пучок

$$
\mathscr{D}(k)=\mathscr{D}+T_{k},
$$

где

$$
T_{k}=\left(\begin{array}{cc}
0 & \pi i\left(k_{1}-i k_{2}\right) \\
-\pi i\left(k_{1}+i k_{2}\right) & 0
\end{array}\right),
$$

который аналитичен по $k_{1}, k_{2}$.

Мы видим, что найти функцию Флоке $\psi$ с квазиимпульсами $k_{1}, k_{2}$ и энергией $E$ - это то же самое, что найти периодическое решение $\varphi$ уравнения

$$
\mathscr{D}(k) \varphi=E \varphi .
$$

Мы рассмотрим решения этого уравнения из $L_{2}$.

Выберем такое $E_{0}$, что оператор $\left(\mathscr{D}_{0}-E_{0}\right)$ обратим на $L_{2}$, т.е. существует обратный оператор

$$
\left(\mathscr{D}_{0}-E_{0}\right)^{-1}: L_{2} \rightarrow W_{2}^{1} .
$$

Представим $\varphi$ в виде

$$
\varphi=\left(\mathscr{D}_{0}-E_{0}\right)^{-1} f
$$

и, подставляя это выражение в уравнение

$$
(\mathscr{D}(k)-E) \varphi=0,
$$

получим следующее уравнение:

$$
(1+A(k, E)) f=0, \quad f \in L_{2},
$$


где

$$
A(k, E)=\left(\begin{array}{cc}
U+\left(E_{0}-E\right) & \pi i\left(k_{1}-i k_{2}\right) \\
-\pi i\left(k_{1}+i k_{2}\right) & V+\left(E_{0}-E\right)
\end{array}\right)\left(\mathscr{D}_{0}-E_{0}\right)^{-1}=B(k, E)\left(\mathscr{D}_{0}-E_{0}\right)^{-1} .
$$

Окончательно проблема существования функции Флоке с квазиимпульсами $k$ и энергией $E$ сводится к разрешимости уравнения

$$
(1+A(k, E)) f=0
$$

в $L_{2}$. Заметим, что оператор $A(k, E)$ разлагается в следующую цепочку операторов:

$$
L_{2} \stackrel{\left(\mathscr{D}_{0}-E\right)^{-1}}{\longrightarrow} W_{2}^{1} \stackrel{\text { вложение }}{\longrightarrow} L_{2} \stackrel{\text { умножение }}{\longrightarrow} L_{2} .
$$

Первое отображение непрерывно, второе - компактно, и в предположении, что потенциалы $U$ и $V$ ограничены, третье отображение - умножение на $B(k, E)$ непрерывно. Следовательно, мы имеем

ПреДЛОЖЕНИЕ 7. Для заданных ограниченных потенииалов $U$ u $V$ аналитический пучок операторов $A(k, E): L_{2} \rightarrow L_{2}$ состоит из компактных операторов.

Теперь мы можем использовать теорему Келдыша [49], [50], которая является альтернативой Фредгольма для аналитических операторных пучков вида $[1+A(\mu)]$, где оператор $A(\mu)$ компактен для каждого $\mu$. Она утверждает, что

резольвента пучка $[1+A(\mu)]: H \rightarrow H$, где $A(\mu)$ - аналитический пучок компактных операторов, является мероморфной функцией по $\mu$; ее особенности отвечают решениям уравнения $(1+A(\mu)) f=0$ и образуют аналитическое подмножество $Q$ в пространстве параметров $\mu$.

В дальнейшем мы будем рассматривать только функции Флоке с $E=0$.

Для оператора $\mathscr{D}$ с потенциалами $U, V$ мы имеем $\mu=(k, E) \in \mathbb{C}^{3}$, и мы положим

$$
Q_{0}(U, V)=Q \cap\{E=0\} .
$$

Это множество инвариантно относительно сдвигов на векторы из двойственной решетки $\Lambda^{*} \subset \mathbb{R}^{2}=\mathbb{C}$ :

$$
k_{1} \rightarrow k_{1}+\eta_{1}, \quad k_{2} \rightarrow k_{2}+\eta_{2} .
$$

Напомним, что двойственная решетка состоит из таких векторов $\eta=\eta_{1}+i \eta_{2}$, что $(\eta, \gamma)=\eta_{1} \gamma^{1}+\eta_{2} \gamma^{2}$ для всех $\gamma=\gamma^{1}+i \gamma^{2} \in \Lambda$.

Спектральная кривая определяется как

$$
\Gamma=Q_{0}(U, V) / \Lambda^{*} .
$$

ЗАмечАНИЕ. Легко заметить, что композиция оператора

$$
(\mathscr{D}(k)-E)^{-1}=\left(\mathscr{D}_{0}-E_{0}\right)^{-1}(1+A(k, E))^{-1}: L_{2} \rightarrow W_{2}^{1}
$$

и канонического вложения $W_{2}^{1} \rightarrow L_{2}$ - это резольвента $R(k, E)$ оператора

$$
\mathscr{D}(k)=\mathscr{D}+\left(\begin{array}{cc}
0 & \pi i\left(k_{1}-i k_{2}\right) \\
-\pi i\left(k_{1}+i k_{2}\right) & 0
\end{array}\right) .
$$

Пересечение множества полюсов резольвенты $R(k, E)$ с плоскостью $E=0$ есть $Q_{0}(U, V)$. 
Мы приходим к следующим определениям:

а) спектральная кривая Г оператора $\mathscr{D}$ с потенциалами $U$ и $V$ - это комплексная кривая $Q(U, V) / \Lambda^{*}$, рассматриваемая с точностью до биголоморфной эквивалентности;

б) на Г определено мультипликаторное отображение, которое является локальным вложением в точке общего положения:

$$
\mathscr{M}: \Gamma \rightarrow \mathbb{C}^{2}: \mathscr{M}(k)=\left(\mu_{1}, \mu_{2}\right)=\left(e^{2 \pi i\left(k, \gamma_{1}\right)}, e^{2 \pi\left(k, \gamma_{2}\right)}\right),
$$

где $\gamma_{1}, \gamma_{2}$ - образующие $\Lambda \subset \mathbb{C}$ и $\left(k, \gamma_{j}\right)=k_{1} \operatorname{Re} \gamma_{j}+k_{2} \operatorname{Im} \gamma_{j}, j=1,2 ;^{5}$

в) каждой точке из $\Gamma$ сопоставляется пространство функций Флоке с данными мультипликаторами; размерность таких пространств в общем случае увеличивается в сингулярных точках из Г.

ПреДЛОЖЕНИЕ 8. Пустъ $k=\left(k_{1}, k_{2}\right)$ - квазиимпульсы функиии Флоке опеpamopa $\mathscr{D}$.

1) $Е с л и ~ U=\bar{V}$, то $\Gamma$ допускает антиголоморфную инволющию $\tau: k \rightarrow-\bar{k}$.

2) Если $U=\bar{U}$ и $V=\bar{V}$, то $\Gamma$ допускает антиголоморфную инволющию $k \rightarrow \bar{k}$.

3) Если $U=\bar{U}=V$, то композиция инволюций из 1) и 2) дает голоморфную инволюиию $\sigma: k \rightarrow-k$.

Такие условия обычны для спектральных кривых (см., например, случай потенциального оператора Шрёдингера в [38], [39]) и для оператора Дирака объяснены в [5], [40], [41]. Простейшее из них - первое, которое доказывается с помощью следующей очевидной леммы.

Лемма 2. Если $U=\bar{V}$, то преобразование $\varphi \rightarrow \varphi^{*}$ вида (4) переводит функиии Флоке в функиии Флоке, изменяя мультипликаторы следующим образом: $k \rightarrow-\bar{k}$.

Обозначим через $\Gamma_{\mathrm{nm}}$ нормализацию Г. Риманова поверхность Г не алгебраическая, но является комплексным пространством, для которых существование нормализации было доказано в [51]. Поскольку мы находимся в одномерной ситуации все сингулярные точки изолированы и нормализация имеет следующий вид:

1) если точка $P \in \Gamma$ приводима, т.е. несколько ветвей Г пересекаются в $P$, то эти ветви расклеиваются;

2 ) для неприводимой сингулярной точки $P$ нормализация $\Gamma_{\mathrm{nm}} \rightarrow \Gamma$ является локальным гомеоморфизмом около $P$ и записывается в форме рядов от локальных параметров

$$
k_{1}=t^{a}+\cdots, \quad k_{2}=t^{b}+\cdots, \quad a>1, \quad b>1 .
$$

Здесь $t$ - локальная координата около $P$ на $\Gamma_{\mathrm{nm}}$.

Если приводимых сингулярных точек нет, то отображение нормализации $\Gamma_{\mathrm{nm}} \rightarrow \Gamma$ является гомеоморфизмом.

\footnotetext{
5Это отображение зависит от выбора образующих $\gamma_{1}, \gamma_{2}$. Если базис $\gamma_{1}, \gamma_{2}$ заменить на другой базис $\widetilde{\gamma}_{1}=a \gamma_{1}+b \gamma_{2}, \widetilde{\gamma}_{2}=c \gamma_{1}+d \gamma_{2}$, то $\mathscr{M}=\left(\mu_{1}, \mu_{2}\right)$ преобразуется по формуле
}

$$
\mathscr{M} \rightarrow \widetilde{\mathscr{M}}=\left(\mu_{1}^{a} \mu_{2}^{b}, \mu_{1}^{c} \mu_{2}^{d}\right) \text {. }
$$


Род комплексной кривой $\Gamma_{\text {nm }}$ называется геометрическим родом кривой $Г$ и обозначается $p_{g}(\Gamma)$. Говорят, что оператор - конечнозонный (на нулевом уровне энергии), если $p_{g}(\Gamma)<\infty$.

Аналог арифметического рода для $Г$, который входит в теоремы типа Римана-Роха, всегда бесконечен: $p_{a}(\Gamma)=\infty$.

Мы имеем

несингулярные точки нормализованной спектральной кривой $\Gamma_{\mathrm{nm}}$ параметризуют (с точностью до множителей) функции Флоке $\psi$, $\mathscr{D} \psi=0$. В отличие от $\Gamma$ взаимная однозначность такой параметризации нарушается только в конечном числе сингулярных точек. ${ }^{6}$

В п. 4.7 мы покажем, что если род $\Gamma_{\mathrm{nm}}$ конечен, то лучше заменить $\Gamma_{\mathrm{nm}}$ на кривую $\Gamma_{\psi}$, чье определение использует функцию Бейкера-Ахиезера оператоpa $\mathscr{D}$.

ПРИМЕР. СПЕКТРАЛЬНАЯ КРИВАЯ ПРИ $U=V=0$ (СВОБОДНЫЙ ОПЕРАтОР). Для простоты предположим, что $\Lambda=\mathbb{Z}+i \mathbb{Z}$. Функции Флоке имеют вид

$$
\psi^{+}=\left(e^{\lambda_{+} z}, 0\right), \quad \psi^{-}=\left(0, e^{\lambda_{-} \bar{z}}\right)
$$

и параметризуются парой комплексных прямых с параметрами $\lambda_{+}$и $\lambda_{-}$. Эти комплексные прямые образуют нормализованную спектральную кривую $\Gamma_{\mathrm{nm}}$. Так как она имеет конечный род, мы компактифицируем ее парой точек на бесконечности таких, что в них $\psi$ имеет экспоненциальные сингулярности. Квазиимпульсы этих функций равны

$$
\begin{array}{cc}
k_{1}=\frac{\lambda_{+}}{2 \pi i}+n_{1}, & k_{2}=\frac{\lambda_{+}}{2 \pi}+n_{2} \quad \text { для } \quad \psi^{+}, \\
k_{1}=\frac{\lambda_{-}}{2 \pi i}+m_{1}, & k_{2}=-\frac{\lambda_{-}}{2 \pi}+m_{2} \quad \text { для } \quad \psi^{-},
\end{array}
$$

где $m_{j}, n_{j} \in \mathbb{Z}$. Функции $\psi^{+}$и $\psi^{-}$имеют одинаковые мультипликаторы в точках

$$
\lambda_{+}^{m, n}=\pi(n+i m), \quad \lambda_{-}^{m, n}=\pi(n-i m), \quad m, n \in \mathbb{Z},
$$

которые образуют резонансные пары. Комплексная кривая Г получается из двух комплексных прямых попарным отождествлением точек из резонансных пар.

ЗАМЕЧАНИЕ. СПЕКТРАЛЬНАЯ КРИВАЯ И УРАВНЕНИЕ КАДОМЦЕВА-ПЕТВИАшвили. Мы изложили выше схему, которую мы использовали для определения спектральных кривых дифференциальных операторов с периодическими коэффициентами в 1985 году (эта статья никогда не была опубликована, хотя ссылка на нее сделана в [52]). Очень похожая схема, как мы узнали позже, была использована Кучментом [53] (см. также [54]). Но одно наблюдение, касающееся уравнений Кадомцева-Петвиашвили, сделанное в это время, следует упомянуть. Существует два уравнения Кадомцева-Петвиашвили (КП)

$$
\partial_{x}\left(u_{t}+6 u u_{x}+u_{x x x}\right)=-3 \varepsilon^{2} u_{y y}
$$

\footnotetext{
6Это следует из асимптотического поведения спектральной кривой (см. п. 4.3).
} 
где $\varepsilon^{2}= \pm 1$. При $\varepsilon=i$ оно называется уравнением КП I, а при $\varepsilon=1$ - уравнение КП II. С физической точки зрения эти уравнения существенно различны. Оба уравнения допускают схожие представления в виде " $L, A$ "-пары $\dot{L}=[L, A]$ с оператором $L$ вида

$$
L=\varepsilon \partial_{y}+\partial_{x}^{2}+u \text {. }
$$

Здесь потенциал $u$ двояко-периодичен или, что то же самое, определен на торе $\mathbb{R}^{2} / \Lambda$. Свободный оператор равен $L_{0}=\varepsilon \partial_{y}-\partial^{2}$ и, чтобы доказать существование спектральной кривой по указанной выше схеме, мы должны рассмотреть обратный оператор

$$
\left(L_{0}-E_{0}\right)^{-1}: L_{2} \rightarrow W_{2}^{2,1}
$$

где $W_{2}^{2,1}$ - пространство таких функций на торе, что $u, u_{x}, u_{x x}$ и $u_{y}$ принадлежат $L_{2}$. Для упрощения вычислений мы рассмотрим случай, когда $\Lambda$ порождена векторами $(2 \pi, 0)$ и $\left(0,2 \pi \tau^{-1}\right)$. Тогда базис Фурье в $L_{2}$ образован функциями

$$
e^{i(k x+l \tau y)}, \quad k, l \in \mathbb{Z} .
$$

В этом базисе оператор $\left(L_{0}-E_{0}\right)$ диагонален и мы имеем

$$
\left(L_{0}-E_{0}\right) e^{i(k x+l \tau y)}=\left(i \varepsilon l \tau-k^{2}-E_{0}\right) e^{i(k x+l \tau y)} .
$$

Так как $\varepsilon=1$ для KP II, мы получаем при $E_{0}>0$ ограниченный оператор

$$
\left(L_{0}-E_{0}\right)^{-1} e^{i(k x+l \tau y)}=\frac{1}{i l \tau-k^{2}-E_{0}} e^{i(k x+l \tau y)} .
$$

Легко проверить, что если $\varepsilon=i$, то для любого значения $E_{0}$ либо оператор $\left(L_{0}-E_{0}\right)$ не обратим, либо обратный к нему не ограничен. Это верно для любой решетки $\Lambda$. Из этих рассуждений можно вывести, что для оператора $L=i \partial_{y}+$ $\partial_{x}^{2}+u$ спектральная кривая не существует. Для оператора теплопроводности $L=\partial_{y}+\partial_{x}^{2}+u$ она существует и сохраняется уравнением КП II.

Спектральная кривая двумерного периодического оператора на нулевом уровне энергии была впервые введена в работе Дубровина, Кричевера и Новикова [4] для случая оператора Шрёдингера, где показано, что

1) периодический оператор, который конечнозонен на нулевом уровне энергии, восстанавливается по некоторым алгебраическим данным, включающим эту кривую; ${ }^{7}$

2) эта кривая является первым интегралом деформаций оператора $L$, заданных “ $L, A, B$ ”-тройками.

ПреДЛОЖЕНИЕ 9 [4]. Пустъ $L$ - двумерный периодический оператор, $\Gamma$ его спектральная кривая и $\mathscr{M}$ - мультипликаторное отображение.

Пусть дано эволющионное уравнение

$$
\frac{\partial L}{\partial t}=[L, A]-B L,
$$

где оператор $A$ также периодический. Тогда эта деформачия оператора $L$ сохраняет $\Gamma$ и $\mathscr{M}$.

\footnotetext{
7 Для оператора Дирака $\mathscr{D}$ см. формулу восстановления (51) и ее вывод в [55] и в п. 4.7.
} 
Этот результат обобщает закон сохранения спектральной кривой одномерного оператора $L$ при его деформации, заданной “ $L, A$ "-парой $\frac{\partial L}{\partial t}=[L, A]$ (это впервые было установлено Новиковым для периодического уравнения КдФ в $[56])$.

Это предложение вытекает из уравнения деформаций $\psi_{t}+A \psi=0$ для функций Флоке, которое сохраняет мультипликаторы (см. П. 3.1 и уравнение (26)). Сохранение нулевого уровня спектра для общих операторов впервые было указано Манаковым в работе [57], где и были введены “ $L, A, B$ "-тройки.

СледСтвиЕ 2. Спектралъная кривая Г и мулътипликаторное отображение $\mathscr{M}$ периодического оператора Дирака $\mathscr{D}$ сохраняются модифицированными уравнениями Веселова-Новикова и уравнениями Дэви-Стюартсона.

Для уравнений мКдФ мы имеем две спектральные кривые: $Г$, определенную для двумерного оператора Дирака, и $\Gamma^{\prime}$, определенную для одномерного оператора $L_{\mathrm{mKdV}}$, входящего в задачу Захарова-Шабата (см. п. 3.2 ) и в представление в виде " $L, A$ "-пары для уравнения мКдФ. Эти комплексные кривые связаны каноническим разветвленным двулистным накрытием $\Gamma \rightarrow \Gamma_{0}[40]$ и обе они сохраняются уравнением мКдФ. Комплексная кривая $\Gamma_{0}$ однозначно восстанавливается по интегралам Крускала-Миуры $H_{k}, k=1,2, \ldots$, которые также являются первыми интегралами уравнения мКдФ.

4.3. Асимптотическое поведение спектральной кривой. Спектральная кривая оператора $\mathscr{D}$ является возмущением спектральной кривой свободного оператора $\mathscr{D}_{0}$. Хотя возмущение может быть достаточно сильным в ограниченной области $|k| \leqslant C$, вне этой области оно выражается в преобразовании двойных точек, отвечающих резонансным парам, в ручки. Более того, размер ручек убывает при $|k| \rightarrow \infty$ и оценивается в терминах возмущения.

Таким образом, мы имеем:

1) компактную часть $\Gamma_{0}=Q_{0} \cap\{|k| \leqslant C\}$, чья граница состоит из пары окружностей;

2 ) комплексную кривую $\Gamma_{\infty}$, полученную из плоскостей $k_{1}=i k_{2}$ и $k_{1}=-i k_{2}$ удалением из них областей $\{|k| \leqslant C\}$ и преобразованием некоторых двойных точек, отвечающих резонансным парам, в ручки;

3) $\Gamma_{0}$ и $\Gamma_{\infty}$ склеиваются вдоль их границ;

4) Г имеет два конца, на которых $\mathscr{M}(\Gamma)$ асимптотически ведет себя так же, как в случае свободного оператора.

Данная комплексная кривая получается из спектральной кривой Г расклейкой двойных точек, которые отвечали резонансным парам и сохранились при возмущении. Мы будем обозначать ее опять через Г.

Оператор называется конечнозонным (на нулевом уровне энергии), если при возмущении $\mathscr{D}_{0} \rightarrow \mathscr{D}$ только конечное число двойных точек преобразовалось в ручки.

Эта картина типична для теории солитонов, где спектральная кривая оператора с потенциалами является возмущением спектральной кривой соответствующего свободного оператора и при этом возмущение мало при больших квазиимпульсах. Она была строго обоснована Кричевером для двумерного оператора Шрёдингера с использованием асимптотических методов [52]. В [10] мы предложили обосновать эту картину для оператора Дирака с помощью тех же 
самых методов и сформулировали предполагаемое утверждение как Предтеорему.

Теория спектральных кривых инициировала развитие аналитической теории римановых поверхностей (не только гиперэллиптических) бесконечного рода [58], [59].

В [5] Шмидт предложил другой подход к обоснованию такого асимптотического поведения спектральной кривой. Этот подход основан на его результате о существовании спектральных кривых для операторов Дирака с $L_{2}$-потенциалами и непрерывном поведении этих кривых для слабо сходящихся последовательностей потенциалов.

Tеорема 10 [5]. При $U, V \in L_{2}\left(T^{2}\right)$ уравнение

$$
\mathscr{D}(k) \varphi=\left(\mathscr{D}+T_{k}\right) \varphi=E \varphi,
$$

где $k \in \mathbb{C}^{2}, E \in \mathbb{C}$, имеет решение в $L_{2}$, если и только если $(k, E) \in Q$, где $Q$ - аналитическое подмножество в $\mathbb{C}^{3}$. Это подмножество $Q$ образовано полюсами операторного пучка

$$
\left(1+A_{U, V}(k, E)\right)^{-1} L_{2} \rightarrow L_{2},
$$

где оператор $A_{U, V}(k, E)$ полиномиален по $k, E$. Более того, если

$$
U_{n}, V_{n} \stackrel{\text { слабо }}{\longrightarrow} U_{\infty}, V_{\infty}
$$

${ }_{8}\left\{\|U\|_{2 ; \varepsilon} \leqslant C,\|V\|_{2 ; \varepsilon} \leqslant C\right\},{ }^{8} m o$

$$
\left\|A_{U_{n}, V_{n}}(k, E)-A_{U_{\infty}, V_{\infty}}(k, E)\right\|_{2} \rightarrow 0
$$

равномерно около каждого $k \in \mathbb{C}^{2}$.

Мы излагаем доказательство этой теоремы в приложении 1. Вернемся к асимптотическому поведению спектральной кривой.

Сначала укажем следующее тождество, которое проверяется прямыми вычислениями:

$$
\begin{gathered}
\left(\begin{array}{cc}
e^{-a} & 0 \\
0 & e^{-b}
\end{array}\right)\left(\mathscr{D}_{0}+\left(\begin{array}{cc}
U & 0 \\
0 & V
\end{array}\right)+T_{k}\right)\left(\begin{array}{cc}
e^{b} & 0 \\
0 & e^{a}
\end{array}\right) \\
=\mathscr{D}_{0}+\left(\begin{array}{cc}
e^{b-a} U & 0 \\
0 & e^{a-b} V
\end{array}\right)+T_{k}+\left(\begin{array}{cc}
0 & a_{z} \\
-b_{\bar{z}} & 0
\end{array}\right)
\end{gathered}
$$

для всех гладких функций $a, b: \mathbb{C} \rightarrow \mathbb{C}$.

Для каждого $\kappa=\left(\kappa_{1}, \kappa_{2}\right) \in \Lambda^{*} \subset \mathbb{C}$ определим $\Lambda$-периодические функции

$$
\psi_{ \pm \kappa}(z, \bar{z})=e^{ \pm 2 \pi i\left(\kappa_{1} x+\kappa_{2} y\right)}
$$

и выберем функции $a(z, \bar{z})$ и $b(z, \bar{z})$ вида

$$
a(z, \bar{z})=2 \pi i\left(\alpha_{1} x+\alpha_{2} y\right), \quad b(z, \bar{z})=2 \pi i\left(\left(\alpha_{1}-\kappa_{1}\right) x+\left(\alpha_{2}-\kappa_{2}\right) y\right),
$$

\footnotetext{
${ }^{8}$ Напомним, что последовательность $\left\{u_{n}\right\}$ в гильбертовом пространстве $H$ слабо сходится к $u_{\infty}: u_{n} \stackrel{\text { слабо }}{\longrightarrow} u_{\infty}$, если для каждого $v \in H$ мы имеем $\lim _{n \rightarrow \infty}\left\langle u_{n}, v\right\rangle=\left\langle u_{\infty}, v\right\rangle$, где $\langle u, v\rangle-$ гильбертово произведение в $H$.
} 
где

$$
\alpha(\kappa)=\left(\alpha_{1}, \alpha_{2}\right)=\left(\frac{\kappa_{1}+i \kappa_{2}}{2}, \frac{-i \kappa_{1}+\kappa_{2}}{2}\right) .
$$

Следующие равенства очевидны: $e^{b-a}=\psi_{-\kappa}, a_{z}=b_{\bar{z}}=0$. Вместе с (43) это влечет

ПрЕДЛОЖЕНИЕ 10 [5]. Если $\varphi \in L_{2}$ удовлетворяет уравнению

$$
\left[\mathscr{D}_{0}+\left(\begin{array}{cc}
\psi_{-\kappa} U & 0 \\
0 & \psi_{\kappa} V
\end{array}\right)+T_{k}\right] \varphi=0
$$

то $\varphi^{\prime}=\left(\begin{array}{cc}\psi_{-\kappa} & 0 \\ 0 & 1\end{array}\right) \varphi \in L_{2}$ удовлетворяет уравнению

$$
\left(\mathscr{D}+T_{k+\alpha}\right) \varphi^{\prime}=0 .
$$

Следовательно,

$$
Q_{0}\left(\psi_{-\kappa} U, \psi_{\kappa} V\right)=Q_{0}(U, V)+\alpha(\kappa) \quad \text { для всех } \quad \kappa \in \Lambda^{*}
$$

(здесь правая часть обозначает множество $Q_{0}(U, V)$, сдвинутое на $\alpha$ ).

Функции $\psi_{\kappa}, \kappa \in \Lambda^{*}$, образуют базис Фурье в $L_{2}$. Отображение $U \rightarrow \widehat{U}=$ $\psi_{\kappa} U, U=\sum_{\nu \in \lambda^{*}} U_{\nu} \psi_{\nu}$, сдвигает коэффициенты Фурье функции $U: \widehat{U}_{\nu}=U_{\nu-\kappa}$. Следовательно, мы имеем

$$
\psi_{\kappa} U \stackrel{\text { слабо }}{\longrightarrow} 0 \quad \text { при } \quad|\kappa| \rightarrow \infty
$$

Теорема 10 (см. приложение 1) и предложение 10 влекут, что в малых ограниченных окрестностях $O(k)$ точек $k \in \mathbb{C}^{2}$ при больших $|\kappa|$ пересечение $Q_{0}(U, V)$ с $O(k)+\alpha(\kappa)$ очень близко к пересечению $Q_{0}(0,0)$ с $O(k)$ :

$$
Q_{0}(U, V) \cap[O(k)+\alpha(\kappa)] \approx Q_{0}(0,0) \cap O(k) \quad \text { при } \quad|\kappa| \rightarrow \infty .
$$

Мы заключаем, что асимптотически при $|k| \rightarrow \infty$ спектральная кривая оператора $\mathscr{D}$ ведет себя как спектральная кривая свободного оператора $\mathscr{D}_{0}$ на $L_{2}$.

При $U=V=0$ спектральная кривая $\Gamma$ биголоморфно эквивалентна паре плоскостей (комплексных прямых), заданных в $\mathbb{C}^{2}$ уравнениями

$$
k_{2}=i k_{1}, \quad k_{2}=-i k_{1}
$$

и склеенных по бесконечному числу пар точек, отвечающих так называемым резонансным парам

$$
\left(k_{1}=\frac{\bar{\gamma}_{1} n-\bar{\gamma}_{2} m}{\bar{\gamma}_{1} \gamma_{2}-\gamma_{1} \bar{\gamma}_{2}}, k_{2}=i k_{1}\right) \leftrightarrow\left(k_{1}=\frac{\gamma_{1} n-\gamma_{2} m}{\bar{\gamma}_{1} \gamma_{2}-\gamma_{1} \bar{\gamma}_{2}}, k_{2}=-i k_{1}\right),
$$

где $m, n \in \mathbb{Z}$. Более того, эти плоскости естественно пополняются парой точек $\infty_{ \pm}$, лежащих на бесконечности и получающихся в пределе $\left(k_{1}, \pm i k_{1}\right) \rightarrow$ $\infty_{ \pm}$при $k_{1} \rightarrow \infty$. Около точек общего положения определено двойное накрытие $\Gamma \rightarrow \mathbb{C}:\left(k_{1}, k_{2}\right) \rightarrow k_{1}$. Согласно предложению 10 , мы имеем 
СлеДСтвиЕ 3. Для оператора Дирака с $L_{2}$-потенциалами $\mathscr{M}(\Gamma)$ при достаточно больших $|k|$ асимптотически ведет себя как

$$
k_{2} \approx \pm i k_{1}
$$

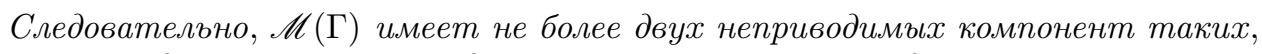
что каждая компонента содержит по крайней мере один из асимптотических концов.

Граница на число неприводимых компонент очевидна, поскольку другие компоненты должны быть локализованы в ограниченной области из $\mathbb{C}^{2}$, что невозможно для одномерных аналитических множеств.

Тем самым, мы приходим к определению, согласованному с используемым в теории конечнозонного интегрирования [4], [60]:

если спектральная кривая Г оператора $\mathscr{D}$ имеет конечный род, то этот оператор конечнозонен и мы называем пополнение Г парой бесконечно удаленных точек $\infty_{ \pm}$спектральной кривой конечнозонного оператора.

Мы закончим процедурой, позволяющей восстановить значение

$$
\int_{\mathbb{C} / \Lambda} U V d x \wedge d y
$$

по $(\Gamma, \mathscr{M})$, когда $\Gamma$ конечного рода. Около асимптотического конца $k_{2} \approx i k_{1}$ введем локальный параметр $\lambda_{+}^{-1}$ такой, что мультипликаторы ведут себя следующим образом:

$$
\mu(\gamma)=\lambda_{+} \gamma+\frac{C_{0} \bar{\gamma}}{\lambda_{+}}+O\left(\lambda_{+}^{-2}\right) .
$$

Тогда

$$
\int_{\mathbb{C} / \Lambda} U V d x \wedge d y=-C_{0} \cdot(\operatorname{Area}(\mathbb{C} / \Lambda))
$$

(см. [61], [10] для случая $U=V$ ).

Аналогичная формула для площади минимальных торов в $S^{3}$ была выведена Хитчиным в [62].

Эта формула дает основание рассматривать пару $(\Gamma, \mathscr{M})$ как обобщение функционала Уиллмора. Впервые это обсуждалось для торов вращения в [46]. В этом случае спектральная кривая восстанавливается по бесконечному числу величин, известных как интегралы Крускала-Миуры.

4.4. Спектральные кривые торов. Для тора $\Sigma$, погруженного в трехмерную группу Ли $G=\mathbb{R}^{3}, S U(2)=S^{3}$, Nil или $\widetilde{S L_{2}}$, и его представления Вейерштрасса рассмотрим спектральную кривую Г оператора $\mathscr{D}$, входящего в это представление.

Мы назовем ее спектральной кривой тора $\Sigma$.

Она определена для всех гладких торов, а не только для интегрируемых (см. п. 4.6). Это определение изначально было введено для торов в $\mathbb{R}^{3}$ в [3] и для торов в $S^{3}$ в [6] в его связи с физическим объяснением гипотезы Уиллмора. Формула (44) показывает, что функционал Уиллмора восстанавливается по Г 
и мультипликаторному отображению $\mathscr{M}$ (по крайней мере в случае, когда Г имеет конечный род).

Это определение не зависит от выбора конформного параметра на торе $\Sigma=$ $\mathbb{R}^{2} / \Lambda$. Мультипликаторное отображение $\mathscr{M}$ зависит от выбора базиса в $\Lambda$, и изменение базиса приводит к простому алгебраическому преобразованию $\mathscr{M}$ (см. (42)).

Определим спектральную кривую для торов в $\mathbb{R}^{4}$.

В [35] мы объяснили, что представление Вейерштрасса для поверхности в $\mathbb{R}^{4}$ неединственно. Потенциалы различных представлений тора связаны формулой

$$
U \rightarrow U \exp (\bar{a}+\overline{b z}-a-b z)
$$

где $\operatorname{Im} b \gamma \in \pi \mathbb{Z}$ для всех $\gamma \in \Lambda$. Мультипликаторное отображение $\mathscr{M}$ зависит от выбора $U$ и при преобразовании (45) изменяется следующим образом:

$$
\mu(\gamma) \rightarrow e^{b \gamma} \mu(\gamma), \quad \gamma \in \Lambda
$$

Как и в случае торов в $\mathbb{R}^{3}$, интеграл от квадрата нормы потенциала $U$ восстанавливается по $(\Gamma, \mathscr{M})$ по той же формуле $(44)$.

Конформная инвариантность функционала Уиллмора привела нас к гипотезе, которую мы обосновали численными экспериментами в [46] и которая очень скоро после ее формулировки была подтверждена в [61]:

ТеОРема 11. Для тора в $\mathbb{R}^{3}$ его спектральная кривая $Г$ и мультипликаторное отображение $\mathscr{M}$ инвариантны относительно конформных преобразований $\overline{\mathbb{R}}^{3}$.

Доказательство из [61] строго работает только для спектральных кривых конечного рода и является следующим. Рассмотрим генераторы конформной группы $S O(4,1)$ и распишем деформационные уравнения для функций Флоке $\varphi$, имеющие вид

$$
\mathscr{D} \delta \varphi+\delta U \cdot \varphi
$$

Достаточно проверить инвариантность только относительно инверсий и даже только для одной из них, поскольку все они попарно сопряжены ортогональными преобразованиями. Выберем следующий генератор для инверсии:

$$
\delta x^{1}=-2 x^{1} x^{3}, \quad \delta x^{2}=-2 x^{2} x^{3}, \quad \delta x^{3}=\left(x^{1}\right)^{2}+\left(x^{2}\right)^{2}-\left(x^{3}\right)^{2},
$$

и вычислим соответствующую вариацию потенциала:

$$
\delta U=\left|\psi_{2}\right|^{2}-\left|\psi_{1}\right|^{2}
$$

где $\psi$ порождает тор. В [61] для этой вариации найдена явная формула в терминах функций, мероморфных на спектральной кривой, для решения уравнения (46). Из этой явной формулы следует, что мультипликаторы сохраняются. Для спектральной кривой конечного рода эти мероморфные функции легко определить. В случае спектральной кривой бесконечного рода необходимо прояснить некоторые аналитические детали, что, по нашему мнению, можно сделать и что зависит от строгого и тщательного изучения асимптотического поведения спектральной кривой.

Другое доказательство теоремы 11 для изотермических торов дано в [10]. Оно геометрическое и работает для спектральных кривых любого рода. 


\section{5. Примеры спектральных кривых.}

ПрОИЗВЕДЕНИЯ ОКРУЖНОСТЕЙ В $\mathbb{R}^{4}$. Рассмотрим торы $\Sigma_{r, R}$, заданные уравнениями

$$
\left(x^{1}\right)^{2}+\left(x^{2}\right)^{2}=r^{2}, \quad\left(x^{3}\right)^{2}+\left(x^{4}\right)^{2}=R^{2} .
$$

Они параметризуются угловыми переменными $x, y$, определенными по модулю $2 \pi: x^{1}=r \cos x, x^{2}=r \sin x, x^{3}=R \cos y, x^{4}=R \sin y$. Конформный параметр, решетка периодов и индуцированная метрика имеют вид

$$
z=x+i \frac{R}{r} y, \quad \Lambda=\left\{2 \pi m+i 2 \pi \frac{r}{R} n: m, n \in \mathbb{Z}\right\}, \quad d s^{2}=r^{2} d z d \bar{z} .
$$

Простыми вычислениями получим формулу для гауссова отображения:

$$
a_{1} / a_{2}=-e^{i(y-x)}, \quad b_{1} / b_{2}=e^{-i(y+x)} .
$$

Применим теорему 4 к отображению

$$
\Sigma_{r, R} \rightarrow\left(b_{1}: b_{2}\right)=\left(\frac{e^{-i(x+y)}}{\sqrt{2}}: \frac{1}{\sqrt{2}}\right) \in \mathbb{C} P^{1} .
$$

Мы имеем $g=\frac{i(x+y)}{2}$,

$$
U=\frac{1}{4}\left(\frac{r}{R}+i\right)
$$

и тор $\Sigma_{r, R}$ задается при представлении Вейерштрасса вектор-функциями

$$
\psi_{1}=\psi_{2}=\frac{1}{\sqrt{2}} \exp \left(-\frac{i(x+y)}{2}\right), \quad \varphi_{1}=-\varphi_{2}=-\frac{r}{\sqrt{2}} \exp \left(\frac{i(y-x)}{2}\right) .
$$

Значения функционала Уиллмора на таких торах даны формулой

$$
\mathscr{W}\left(\Sigma_{r, R}\right)=4 \int_{\Sigma_{r, R}}|U|^{2} d x \wedge d y=\pi^{2}\left(\frac{r}{R}+\frac{R}{r}\right)
$$

и достигают минимума на торе Клиффорда $\Sigma$ в $\mathbb{R}^{4}: \mathscr{W}\left(\Sigma_{r, r}\right)=2 \pi^{2}$.

Спектральная кривая $Г(u)$ оператора Дирака

$$
\mathscr{D}=\left(\begin{array}{cc}
0 & \partial \\
-\bar{\partial} & 0
\end{array}\right)+\left(\begin{array}{cc}
u & 0 \\
0 & \bar{u}
\end{array}\right), \quad u=\text { const }
$$

с постоянным потенциалом $U=u$ является комплексной сферой с парой отмеченных точек (“бесконечностей") $\lambda=0$ и $\lambda=\infty$ :

$$
\Gamma(u)=\mathbb{C} P^{1} .
$$

Нормированная функция Бейкера-Ахиезера (или функция Флоке) равна

$$
\psi(z, \bar{z}, \lambda)=\left(\begin{array}{l}
\psi_{1} \\
\psi_{2}
\end{array}\right)=\frac{\lambda}{\lambda-u} \exp \left(\lambda z-\frac{|u|^{2}}{\lambda} \bar{z}\right)\left(\begin{array}{c}
1 \\
-u / \lambda
\end{array}\right) .
$$

Нормировка состоит в выполнении двух асимптотик

$$
\psi \approx\left(\begin{array}{c}
e^{\lambda_{+} z} \\
0
\end{array}\right) \quad \text { при } \quad \lambda_{+} \rightarrow \infty, \quad \psi \approx\left(\begin{array}{c}
0 \\
e^{\lambda_{-} \bar{z}}
\end{array}\right) \quad \text { при } \quad \lambda_{-} \rightarrow 0
$$

с локальными параметрами $\lambda_{+}=\lambda$ около $\lambda=\infty$ и $\lambda_{-}=-|u|^{2} / \lambda$ около $\lambda=\infty$.

Для торов $\Sigma_{r, R}$ мы имеем 
а) функция $\psi$, порождающая их при представлении (18), равна $\psi(z, \bar{z},-u)$, $u=\frac{1}{4}\left(\frac{r}{R}+i\right)$, и имеет монодромию

$$
\psi(z+2 \pi, \bar{z}-2 \pi i,-u)=\psi\left(z+i 2 \pi \frac{R}{r}, \bar{z}-i 2 \pi \frac{R}{r},-u\right)=-\psi(z, \bar{z},-u)
$$

б) существует в точности четыре точки на спектральной кривой $\Gamma(u)$, для которых функция $\psi(z, \bar{z}, \lambda)$ имеет ту же самую монодромию, что и $\psi(z, \bar{z},-\lambda):$ это $-\lambda= \pm u, \pm \bar{u}$. Более того,

$$
\left(\begin{array}{c}
\psi_{1}(z, \bar{z},-u) \\
\psi_{2}(z, \bar{z},-u)
\end{array}\right)=\left(\begin{array}{c}
-\bar{\psi}_{2}(z, \bar{z}, u) \\
\bar{\psi}_{1}(z, \bar{z}, u)
\end{array}\right)
$$

в) спектральная кривая $\Gamma(u)$ - гладкая.

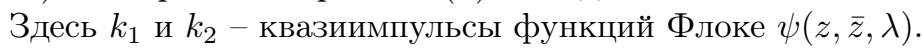

Периодический потенциал $U$ определен с точностью до калибровочного преобразования $(24)$, которое при $b=0$ и $e^{\bar{a}-a}=-\frac{1+i}{\sqrt{2}}$ преобразует потенциал $U$ тора Клиффорда в потенциал

$$
\frac{1}{4}(1+i) \rightarrow \frac{e^{\bar{a}-a}}{4}(1+i)=-\frac{i}{2 \sqrt{2}}
$$

который совпадает с потенциалом этого же тора, рассмотренного как тор в трехмерной сфере $S^{3} \subset \mathbb{R}^{4}$ [10]. Это приводит к следующим вопросам:

1) всегда ли спектральные кривые тора $S^{3} \subset \mathbb{R}^{4}$, определенные для него как для тора в $S^{3}$ и в $\mathbb{R}^{4}$, совпадают?

2) для тора в $S^{3} \subset \mathbb{R}^{4}$ всегда ли потенциал $U$ его представления Вейерштрасса в $\mathbb{R}^{4}$ калибровочно эквивалентен потенциалу его представления Вейерштрасса в $S^{3}: U=\frac{(H-i) e^{\alpha}}{2}$, где $H$ - средняя кривизна тора в $S^{3}$ ?

Положительный ответ на второй вопрос влечет положительный ответ на первый. Мы думаем, что ответы на оба вопроса положительны.

Тор Клиффорда в $\mathbb{R}^{3}$. Тор Клиффорда в $\mathbb{R}^{3}$ является образом тора Клиффорда в $S^{3} \subset \mathbb{R}^{4}$ при стереографической проекции

$$
\left(x^{1}, x^{2}, x^{3}, x^{4}\right) \rightarrow\left(\frac{x^{1}}{1-x^{4}}, \frac{x^{2}}{1-x^{4}}, \frac{x^{3}}{1-x^{4}}\right), \quad \sum_{k}\left(x^{k}\right)^{2}=1 .
$$

Он рассматривается с точностью до конформных преобразований $\overline{\mathbb{R}}^{3}$ и, таким образом, может быть получен как следующий тор вращения: возьмем окружность радиуса $r=1$ в плоскости $x^{1} x^{3}$ такую, что расстояние от ее центра до оси $x^{1}$ равно $R=\sqrt{2}$, тор Клиффорда получается вращением этой окружности вокруг оси $x^{1}$.

Теорема 12 [55]. Функиия Бейкера-Ахиезера оператора Дирака $\mathscr{D}$ с потенциалом

$$
U=\frac{\sin y}{2 \sqrt{2}(\sin y-\sqrt{2})}
$$

есть такая вектор-функиия $\psi(z, \bar{z}, P)$, где $z \in \mathbb{C} u P \in \Gamma$, ито 
1) комплексная кривая $\Gamma$ - это сфера $\mathbb{C} P^{1}=\overline{\mathbb{C}}$ с двумя отмеченными точками $\infty_{+}=(\lambda=\infty), \infty_{-}=(\lambda=0)$, где $\lambda$ - афбинный параметр на $\mathbb{C} \subset \mathbb{C} P^{1}$, и двумя двойными точками, которые получаятся отождествлением точек из следующих пар:

$$
\left(\frac{1+i}{4}, \frac{-1+i}{4}\right) \text { и }\left(-\frac{1+i}{4}, \frac{1-i}{4}\right) ;
$$

2) бункиия $\psi$ мероморфна на $\Gamma \backslash\left\{\infty_{ \pm}\right\}$и имеет в отмеченных точках ("бесконечностях") следующие асимптотики:

$$
\psi \approx\left(\begin{array}{c}
e^{k_{+} z} \\
0
\end{array}\right) \text { при } k_{+}=\lambda \rightarrow \infty ; \quad \psi \approx\left(\begin{array}{c}
0 \\
e^{k_{-} \bar{z}}
\end{array}\right) \text { при } k_{-}=-\frac{|u|^{2}}{\lambda} \rightarrow \infty,
$$

где $u=\frac{1+i}{4} u k_{ \pm}^{-1}-$ локальные параметры около $\infty_{ \pm}$;

3) $\psi$ имеет три полюса на $\Gamma \backslash\left\{\infty_{ \pm}\right\}$, которые не зависят от z и лежат в точкаx

$$
p_{1}=\frac{-1+i+\sqrt{-2 i-4}}{4 \sqrt{2}}, \quad p_{2}=\frac{-1+i-\sqrt{-2 i-4}}{4 \sqrt{2}}, \quad p_{3}=\frac{1}{\sqrt{8}} .
$$

При этом геометрический род $p_{g}(\Gamma)$ и арифметический род $p_{a}(\Gamma)$ кривой $Г$ следующие:

$$
p_{g}(\Gamma)=0, \quad p_{a}(\Gamma)=2 .
$$

Функиия Бейкера-Ахиезера удовлетворяет уравнению Дирака $\mathscr{D} \psi=0$ с потенчиалом $U$ вида (47) в каждой точке из $\Gamma \backslash\left\{\infty_{+}, \infty_{-}, p_{1}, p_{2}, p_{3}\right\}$.

Тор Клиффорда строится посредством представления Вейеритрасса (2) и (3) по функции

$$
\psi=\psi\left(z, \bar{z}, \frac{1-i}{4}\right)
$$

Показано, что функция $\psi$ имеет вид

$$
\begin{aligned}
& \psi_{1}(z, \bar{z}, \lambda)=e^{\lambda z-\frac{|u|^{2}}{\lambda} \bar{z}}\left(q_{1} \frac{\lambda}{\lambda-p_{1}}+q_{2} \frac{\lambda}{\lambda-p_{2}}+\left(1-q_{1}-q_{2}\right) \frac{\lambda}{\lambda-p_{3}}\right), \\
& \psi_{2}(z, \bar{z}, \lambda)=e^{\lambda z-\frac{|u|^{2}}{\lambda} \bar{z}}\left(t_{1} \frac{p_{1}}{p_{1}-\lambda}+t_{2} \frac{p_{2}}{p_{2}-\lambda}+\left(1-t_{1}-t_{2}\right) \frac{p_{3}}{p_{3}-\lambda}\right),
\end{aligned}
$$

где $u=\frac{1+i}{4}$ и функции $q_{1}, q_{2}, t_{1}, t_{2}$ зависят только от $y$ и $2 \pi$-периодичны по $y$. Они находятся из следующих условий:

$$
\psi\left(z, \bar{z}, \frac{1+i}{4}\right)=\psi\left(z, \bar{z}, \frac{-1+i}{4}\right), \quad \psi\left(z, \bar{z},-\frac{1+i}{4}\right)=\psi\left(z, \bar{z}, \frac{1-i}{4}\right) .
$$

4.6. Спектральные кривые интегрируемых торов. Говорят, что поверхность интегрируема, если уравнения Гаусса-Кодацци являются условием совместности

$$
\left[\partial_{x}-A(\lambda), \partial_{y}-B(\lambda)\right]=0
$$

линейных задач

$$
\partial_{x} \varphi=A(\lambda) \varphi, \quad \partial_{y} \varphi=B(\lambda) \varphi,
$$


где $A$ и $B$ - ряды Лорана от спектрального параметра $\lambda$. Также подразумевается, что вхождение $\lambda$ в это представление нетривиально. Для получения явных решений уравнения нулевой кривизны (48) можно использовать весь аппарат теории солитонов и, в частности, теории интегрируемых гармонических отображений, начало развитию которой было положено статьями [63]-[65] и которая интенсивно развивалась в последние 30 лет (ее современное состояние представлено в [66]-[68]). Наиболее полный список интегрируемых поверхностей в $\mathbb{R}^{3}$ дан в [69] (см. также [70]).

Эта теория хорошо работает для сфер, когда достаточно использовать алгебраическую геометрию комплексных рациональных кривых, и для торов, когда явные формулы для поверхностей выводятся в терминах тэта-функций на некоторых римановых поверхностях. Для поверхностей большего рода теория интегрируемых систем не привела к существенному прогрессу. Это, возможно, имеет серьезные причины в том, что среди замкнутых поверхностей только торы допускают плоские метрики.

Спектральные кривые интегрируемых торов возникают как спектральные кривые операторов, входящих в эти вспомогательные линейные задачи. Эти комплексные кривые (римановы поверхности) служат для построения явных формул для торов в терминах тэта-функций этих римановых поверхностей.

Оказывается, это не случайно и эти спектральные кривые интегрируемых торов являются просто частными случаями общих спектральных кривых, определенных в п. 4.4 для всех торов (не только интегрируемых).

В [10] мы доказали такое совпадение (по модулю дополнительных неприводимых компонент) для торов постоянной средней кривизны и изотермических торов в $\mathbb{R}^{3}$ и для минимальных торов в $S^{3}$. Следствие 3 исключает существование дополнительных компонент.

А) Торы Постоянной средней кривизны в $\mathbb{R}^{3}$. По теореме Ру-Вилмса гауссово отображение поверхности в $\mathbb{R}^{3}$ является гармоническим тогда и только тогда, когда поверхность имеет постоянную среднюю кривизну [71]. Согласно уравнениям Гаусса-Кодацци, это эквивалентно тому, что дифференциал Хопфа $A d z^{2}$ голоморфен:

$$
A_{\bar{z}}=0 .
$$

На сфере голоморфный квадратичный дифференциал равен нулю, и по теореме Хопфа сферы постоянной средней кривизны в $\mathbb{R}^{3}$ - это, в точности, круглые сферы (т.е. сферы постоянной кривизны) [72].

Хопф также высказал гипотезу, что все погруженные замкнутые поверхности постоянной средней кривизны в $\mathbb{R}^{3}$ - это, в точности, круглые сферы. Хотя эта гипотеза была подтверждена Александровым для вложенных поверхностей [73], она была опровергнута для поверхностей большего рода. Существование торов постоянной средней кривизны было установлено в начале 1980-х годов Венте с помощью теоремы о неявной функции для банаховых пространств [74]. Первые явные примеры были найдены Абрешем в [75] и анализ этих примеров в [76] указал на связь этой задачи с интегрируемыми системами. Позднее было доказано, что для тора постоянной средней кривизны комплексная кривая Г имеет конечный род [77], это позволило применить функции Бейкера-Ахиезера для получения явных формул для таких торов в терминах тэта-функций римановой поверхности Г (эта программа была реализована Бобенко в [78], [79]). Существование поверхностей постоянной средней кривизны родов, больших единицы, было установлено Капулеасом тоже 
неявными методами [80], [81], и проблема явного описания таких поверхностей остается открытой. Мы отметим, что иная интерпретация поверхностей постоянной средней кривизны в терминах бесконечномерной интегрируемой системы была предложена в [82] и основана на представлении Вейерштрасса.

На торе голоморфный квадратичный дифференциал имеет постоянные коэффициенты (по отношению к конформному параметру $z$ ). Если задан тор постоянной средней кривизны, то гомотетией поврехности и линейной заменой $z \rightarrow a z$ конформного параметра можно привести все к ситуации, когда

$$
A d z^{2}=\frac{1}{2} d z^{2}, \quad H=1 .
$$

В этом случае уравнения Гаусса-Кодацци утверждают, что

$$
u_{z \bar{z}}+\sinh u=0,
$$

где $u=2 \alpha$ и $e^{2 \alpha} d z d \bar{z}$ - метрика на торе. Это уравнение является условием совместности для следующей системы

$$
\left[\frac{\partial}{\partial z}-\frac{1}{2}\left(\begin{array}{cc}
-u_{z} & -\lambda \\
-\lambda & u_{z}
\end{array}\right)\right] \psi=0, \quad\left[\frac{\partial}{\partial \bar{z}}-\frac{1}{2 \lambda}\left(\begin{array}{cc}
0 & e^{-u} \\
e^{u} & 0
\end{array}\right)\right] \psi=0 .
$$

Пусть $\Lambda$ - решетка периодов для тора. Мы рассмотрим линейную задачу

$$
L \psi=\partial_{z} \psi-\frac{1}{2}\left(\begin{array}{cc}
-u_{z} & 0 \\
0 & u_{z}
\end{array}\right) \psi=\frac{1}{2}\left(\begin{array}{cc}
0 & -\lambda \\
-\lambda & 0
\end{array}\right) \psi
$$

Поскольку оператор $L$ - это $(2 \times 2)$-матричный оператор первого порядка, для каждого $\lambda \in \mathbb{C}$ система (49) имеет двумерное пространство $V_{\lambda}$ решений и эти пространства инвариантны относительно операторов трансляций

$$
\widehat{T}_{j} \varphi(z)=\varphi\left(z+\gamma_{j}\right), \quad j=1,2,
$$

где $\gamma_{1}$ и $\gamma_{2}$ - образующие решетки $\Lambda$. Операторы $\widehat{T}_{1}, \widehat{T}_{2}$ и $L$ коммутируют и, следовательно, имеют общие собственные векторы, которые склеиваются в мероморфную функцию $\psi(z, \bar{z}, P)$ на двулистном накрытии

$$
\widehat{\Gamma} \rightarrow \mathbb{C}: P \in \widehat{\Gamma} \rightarrow \lambda \in \mathbb{C},
$$

разветвленном в точках, где операторы $\widehat{T}_{j}$ и $L$ не диагонализируются одновременно. Это - стандартная процедура построения спектральных кривых периодических операторов [56].

Каждой точке $P \in \widehat{\Gamma}$ отвечает единственная (с точностью до постоянного множителя) функция Флоке $\psi(z, \bar{z}, P)$ с мультипликаторами $\mu\left(\gamma_{1}, P\right)$ и $\mu\left(\gamma_{2}, P\right)$. Комплексная кривая $\widehat{\Gamma}$ компактифицируется четырьмя "бесконечно удаленными" точками $\infty_{ \pm}^{1}, \infty_{ \pm}^{2}$ так, что $\infty_{ \pm}^{1}$ проектируются в $\lambda=\infty$ и $\infty_{ \pm}^{2}$ проектируются в $\lambda=0$, и мы можем взять функцию $\psi$, мероморфную на $\widehat{\Gamma}$ со следующими существенными особенностями в "бесконечностях":

$$
\begin{aligned}
& \psi(z, \bar{z}, P) \approx \exp \left(\mp \frac{\lambda z}{2}\right)\left(\begin{array}{c}
1 \\
\pm 1
\end{array}\right) \quad \text { при } \quad P \rightarrow \infty_{ \pm}^{1}, \\
& \psi(z, \bar{z}, P) \approx \exp \left(\mp \frac{\bar{z}}{2 \lambda}\right)\left(\begin{array}{c}
1 \\
\pm 1
\end{array}\right) \quad \text { при } \quad P \rightarrow \infty_{ \pm}^{2} .
\end{aligned}
$$


Мультипликаторы стремятся к $\infty$ при $\lambda \rightarrow 0, \infty$.

Комплексная кривая Г допускает инволюцию, сохраняющую мультипликаторы:

$$
\sigma(\lambda)=-\lambda, \quad\left(\begin{array}{c}
\varphi_{1} \\
\varphi_{2}
\end{array}\right) \rightarrow\left(\begin{array}{c}
\varphi_{1} \\
-\varphi_{2}
\end{array}\right), \quad \sigma\left(\infty_{ \pm}^{1}\right)=\infty_{\mp}^{1}, \quad \sigma\left(\infty_{ \pm}^{2}\right)=\infty_{\mp}^{2} .
$$

Комплексная фактор-кривая $\widehat{\Gamma} / \sigma$ называется спектральной кривой тора постоянной средней кривизны.

Мы имеем

ПреДЛОЖЕНИЕ 11 [10]. Вектор-функиия ч удовлетворяет уравнению (49), если и только если $\psi=\left(\lambda \varphi_{2}, e^{\alpha} \varphi_{1}\right)^{\top}$ удовлетворяет уравнению Дирака $\mathscr{D} \psi=0$ $c U=\frac{H e^{\alpha}}{2}=\frac{e^{\alpha}}{2}$.

Тем самым мы имеем аналитическое отображение Г на спектральную кривую тора общего положения, определенную в п. 4.4, сохраняющее значение мультипликаторов. Отсюда следует, что эти комплексные кривые совпадают с точностью до неприводимых компонент. Вместе со следствием 3 это влечет следующее предложение.

ПРЕДЛОжЕНИЕ 12. Спектральная кривая тора постоянной средней кривизны в $\mathbb{R}^{3}$ совпадает с (общей) спектральной кривой этого тора, определенной в $n .4 .4$.

Б) МинимальныЕ торы в $S^{3}$. Мы рассмотрим единичную сферу в $\mathbb{R}^{4}$ как группу Ли $S U(2)$. Для минимальных поверхностей в $S U(2)$ деривационные уравнения (10) и (11) упрощаются, и мы получаем систему Хитчина [62]

$$
\bar{\partial} \Psi-\partial \Psi^{*}+\left[\Psi^{*}, \Psi\right]=0, \quad \bar{\partial} \Psi+\partial \Psi^{*}=0 .
$$

Из первого уравнения следует, что $S L_{2}$-связность $\mathscr{A}=\left(\partial+\Psi, \bar{\partial}+\Psi^{*}\right)$ на $f^{-1}(T G)$ является плоской. При этом второе уравнение влечет, что эта связность продолжается до аналитического семейства плоских связностей

$$
\mathscr{A}_{\lambda}=\left(\partial+\frac{1+\lambda^{-1}}{2} \Psi, \bar{\partial}+\frac{1+\lambda}{2} \Psi^{*}\right),
$$

где $\mathscr{A}=\mathscr{A}_{1}$ и $\lambda \in \mathbb{C} \backslash\{0\}$. Тем самым мы получаем " $L, A$ "-пару со спектральным параметром и делаем вывод, что эта система интегрируема. Этот трюк является общим для интегрируемых гармонических отображений.

Определим спектральную кривую.

Пусть $\Sigma$ - минимальный тор в $S U(2)$ и $\left\{\gamma_{1}, \gamma_{2}\right\}$ - базис для решетки $\Lambda$. Определим матрицы $H(\lambda)$ и $\widetilde{H}(\lambda) \in S L(2, \mathbb{C})$, которые описывают монодромию связности $\mathscr{A}_{\lambda}$ вдоль замкнутой петли, реализующей $\gamma_{1}$ и $\gamma_{2}$ соответственно. Эти матрицы коммутируют и поэтому имеют общие собственные векторы $\varphi(\lambda, \mu)$, где $\mu$ - корень характеристического уравнения для $H(\lambda)$ :

$$
\mu^{2}-\operatorname{Tr} H(\lambda)+1=0 .
$$

Собственные значения

$$
\mu_{1,2}=\frac{1}{2}\left(\operatorname{Tr} H(\lambda) \pm \sqrt{\operatorname{Tr}^{2} H(\lambda)-4}\right)
$$


определены на римановой поверхности Г, которая является двулистным накрытием $\mathbb{C} P^{1}$, разветвленным в простых нулях функции $\left(\operatorname{Tr}^{2} H(\lambda)-4\right)$ и в 0 и $\infty$ (кратные нули удаляются при нормализации). Эта комплексная кривая Г называется спектральной кривой минимального тора в $S U(2)$ и имеет конечный род.

Выше мы изложили результаты Хитчина, которые верны для всех гармонических торов в $S^{3}$ (это включает оба случая минимальных торов в $S^{3}$ и гармонических гауссовых отображений в $S^{2} \subset S^{3}$ ) [62]. Теперь мы ограничимся минимальными торами в $S^{3}$.

Пусть $\mathscr{D}$ - оператор Дирака, ассоциированный с этим тором, и $\psi^{\prime}-$ спинор, задающий тор посредством представления Вейерштрасса. Пусть

$$
L=\frac{1}{\sqrt{2}}\left(\begin{array}{cc}
\bar{a} & -\bar{b} \\
b & a
\end{array}\right), \quad a=-i \bar{\psi}_{1}^{\prime}+\psi_{2}^{\prime}, \quad b=-i \psi_{1}^{\prime}+\bar{\psi}_{2}^{\prime} .
$$

Мы имеем следующее предложение.

ПредЛОЖЕниЕ 13 [10]. Собственные функции Хитчина ч преобразуются отображением

$$
\varphi \rightarrow \psi=e^{\alpha}\left(\begin{array}{cc}
0 & i \lambda \\
1 & 0
\end{array}\right) \cdot L^{-1} \varphi
$$

в решения уравнения Дирака $\mathscr{D} \psi=0$, отвечающего тору $\Sigma$ в $S^{3}$.

Как и в случае торов постоянной средней кривизны в $\mathbb{R}^{3}$ (см. выше), это предложение вместе со следствием 3 влечет

ПРеДЛОЖениЕ 14. Спектралъная кривая минимального тора в $S^{3}$ совпадает с (общей) спектралъной кривой этого тора, определенной в n. 4.4.

4.7. Сингулярные спектральные кривые. Возмущение свободного оператора можем быть настолько сильным, что у спектральной кривой $Г$ возникнут другие особые точки (кроме резонансных пар). Если $\Gamma_{\mathrm{nm}}-$ алгебраическая кривая, мы выпишем функцию Бейкера-Ахиезера $\psi(z, \bar{z}, P)$ такую, что

1) $\mathscr{D} \psi=0$

2) $\psi$ мероморфна на Г и имеет следующие асимптотики в бесконечно удаленных точках:

$$
\psi \approx\left(\begin{array}{c}
e^{\lambda_{+} z} \\
0
\end{array}\right) \quad \text { при } \quad P \rightarrow \infty_{+}, \quad \psi \approx\left(\begin{array}{c}
0 \\
e^{\lambda_{-} \bar{z}}
\end{array}\right) \quad \text { при } \quad P \rightarrow \infty_{-},
$$

где $\lambda_{ \pm}^{-1}-$ локальные координаты около $\infty_{ \pm}, \lambda_{ \pm}^{-1}\left(\infty_{ \pm}\right)=0$; можно положить $\lambda_{ \pm}=2 \pi i k_{1}$.

Функция $\psi$ образована функциями Флоке $\psi(z, \bar{z}, P)$, взятыми в различных точках спектральной кривой, так, что $\psi$ мероморфна и имеет указанные выше асимптотики. Функция $\psi$ уже “вырисовывает" кривую $\Gamma_{\psi}$, на которой она определена так, что никакая функция Флоке не учитывается дважды в различных точках из $\Gamma_{\psi}$. Имеет место цепочка отображений

$$
\Gamma_{\mathrm{nm}} \rightarrow \Gamma_{\psi} \rightarrow \Gamma
$$

такая, что их композиция является нормализацией кривой Г и первое из них является нормализацией кривой $\Gamma_{\psi}$. Мы имеем очевидные неравенства:

$$
p_{g}(\Gamma)=p_{g}\left(\Gamma_{\psi}\right) \leqslant p_{a}\left(\Gamma_{\psi}\right)<\infty,
$$


где $p_{a}\left(\Gamma_{\psi}\right)$ - арифметический род кривой $\Gamma_{\psi}$, отличающийся от геометрического рода за счет вклада особых точек.

Функцию $\psi$ можно поднять обратно на несингулярную кривую Г, на которой она имеет в точности $p_{a}\left(\Gamma_{\psi}\right)+1$ полюсов (это следует из теории конечнозонного интегрирования). Для оператора Дирака $p_{a}\left(\Gamma_{\psi}\right)$ на единицу меньше, чем "число полюсов нормализованной функции Бейкера-Ахиезера".

Мы приходим к следующему заключению:

а) функция Бейкера-Ахиезера $\psi$ задает риманову поверхность $\Gamma_{\psi}$ в классическом духе работы Римана, как поверхность, на которой естественно определена данная нам функция $\psi$; эта поверхность получается из Г нормализацией сингулярностей только в тех случаях, когда после нормализации размерность пространства функций Флоке в точке уменьшается (например, это случай резонансных пар);

б) в отличие от $\Gamma_{\mathrm{nm}}$, комплексная кривая $\Gamma_{\psi}$ дает взаимно однозначную параметризацию всех функций Флоке (с точностью до постоянных множителей).

Для минимальных торов в $S^{3}$ эта ситуация объясняется детально в [62].

Если мы хотим построить тор конечного спектрального рода в терминах тэта-функций, мы опять должны работать с кривой $\Gamma_{\psi}$, что мы продемонстрировали в п. 4.5 в случае тора Клиффорда.

Следующее определение $\Gamma_{\psi}$ проистекает из теории конечнозонного интегрирования.

Пусть $\mathscr{D}$ - оператор Дирака с двояко-периодическими потенциалами $U$ и $V$, и пусть $\Gamma_{\psi}$ - риманова поверхность (возможно, с особенностями) конечного арифметического рода $p_{a}\left(\Gamma_{\psi}\right)=g$ с двумя отмеченными неособыми точками $\infty_{ \pm}$и такими локальными координатами $k_{ \pm}^{-1}$ около этих точек, что $k_{ \pm}^{-1}\left(\infty_{ \pm}\right)=0$.

Пусть $\psi(z, \bar{z}, P)$ - функция Бейкера-Ахиезера $\psi$, определенная на $\mathbb{C} \times \Gamma_{\psi} \backslash\left\{\infty_{ \pm}\right\}$и такая, что

1) $\psi$ мероморфна по $P$ вне $\infty_{ \pm} \in \Gamma$ и имеет полюсы в $g+1$ неособых точках $P_{1}+\cdots+P_{g+1}$;

2) $\psi$ имеет следующие асимптотики в $\infty_{ \pm}$:

$$
\begin{aligned}
& \psi \approx e^{k_{+} z}\left[\left(\begin{array}{l}
1 \\
0
\end{array}\right)+\left(\begin{array}{l}
\xi_{1}^{+} \\
\xi_{2}^{+}
\end{array}\right) k_{+}^{-1}+O\left(k_{+}^{-2}\right)\right] \quad \text { при } \quad P \rightarrow \infty_{+}, \\
& \psi \approx e^{k_{-} \bar{z}}\left[\left(\begin{array}{l}
0 \\
1
\end{array}\right)+\left(\begin{array}{l}
\xi_{1}^{-} \\
\xi_{2}^{-}
\end{array}\right) k_{-}^{-1}+O\left(k_{-}^{-2}\right)\right] \quad \text { при } \quad P \rightarrow \infty_{-}
\end{aligned}
$$

и удовлетворяет уравнению Дирака $\mathscr{D} \psi=0$ всюду на $\Gamma_{\psi}$ за исключением "бесконечно удаленных точек" $\infty_{ \pm}$и полюсов $P_{1}, \ldots, P_{g+1}$.

Мы скажем, что $\Gamma_{\psi}$ является спектралъной кривой конечнозонного оператора $\mathscr{D}$.

Для дивизора $P_{1}+\cdots+P_{g+1}$ общего положения такая функция единственна и потенциалы восстанавливаются по формулам:

$$
U=-\xi_{2}^{+}, \quad V=\xi_{1}^{-} .
$$

Попытка определить такую риманову поверхность в случае, когда $p_{g}(\Gamma)=$ $\infty$, встречает много аналитических трудностей. 
Мы отсылаем к [55] за более детальным изложением некоторых вопросов, касающихся сингулярных спектральных кривых.

Мы видели в п. 4.5, что для тора Клиффорда $\Sigma_{1,1} \subset \mathbb{R}^{4}$ потенциал постоянен и спектральная кривая является сферой. Более того,

$$
p_{g}(\Gamma)=p_{a}\left(\Gamma_{\psi}\right)=0
$$

Но потенциал его стереографической проекции - тора Клиффорда в $\mathbb{R}^{3}$ равен

$$
U=\frac{\sin x}{2 \sqrt{2}(\sin x-\sqrt{2})},
$$

где $x$ - одна из угловых переменных, и по теореме 12 для оператора с таким потенциалом мы имеем

$$
p_{g}(\Gamma)=0, \quad p_{a}\left(\Gamma_{\psi}\right)=2 .
$$

Следовательно, стереографическая проекция тора Клиффорда из $S^{3}$ в $\mathbb{R}^{3}$ приводит к возникновению особых точек у кривой $\Gamma_{\psi}$.

Это ведет к интересной задаче:

как связаны спектральная кривая тора в единичной сфере $S^{3} \subset$

$\mathbb{R}^{4}$ и спектральная кривая его стереографической проекции?

Мы думаем, что ответ на этот вопрос следующий: потенциалы связаны некоторым преобразованием Бэклунда, приводящим к преобразованию спектральных кривых. Возможно, имеется аналогия с подобным преобразованием для одномерного оператора Шрёдингера, изложенным в [83]. Мы также предполагаем, что ответ на следующий вопрос положительный:

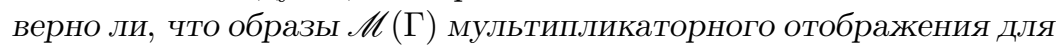

тора в $S^{3}$ и для его стереографической проекции совпадают?

Имеется другая интересная задача:

охарактеризовать спектральные кривые торов в $\mathbb{R}^{3}$ и $\mathbb{R}^{4}$.

Для торов в $\mathbb{R}^{3}$ и $\mathbb{R}^{4}$ ответы должны быть различны. Действительно, еще в [78] было отмечено, что спектральные кривые торов постоянной средней кривизны в $\mathbb{R}^{3}$ должны быть сингулярными (для них это выражается в существовании кратных точек ветвления, которые при нормализации переходят в пары точек, переставляемых гиперэллиптической инволюцией). ${ }^{9}$ Но для тора Клиффорда в $\mathbb{R}^{4}$ спектральная кривая несингулярна.

\section{§ 5. Функционал Уиллмора}

5.1. Поверхности Уиллмора и гипотеза Уиллмора. Функционал Уиллмора для замкнутых поверхностей в $\mathbb{R}^{3}$ определяется как

$$
\mathscr{W}(\Sigma)=\int_{\Sigma} H^{2} d \mu,
$$

${ }^{9} \mathrm{~B}[5]$ показано, что для торов в $\mathbb{R}^{3} \mathscr{M}(\Gamma)$ содержит точку кратности не менее четырех или пару двойных точек, в которых дифференциалы $d k_{1}$ и $d k_{2}$ имеют нули (здесь $k_{1}$ и $k_{2}-$ квазиимпульсы). Мы заметим, что это не означает, что $\Gamma_{\psi}$ удовлетворяет тем же самым условиям: например, для тора Клиффорда в $\mathbb{R}^{3}$ спектральная кривая $\Gamma_{\psi}$ имеет пару двойных точек, в которых $d k_{1}$ и $d k_{2}$ не имеют нулей, и не содержит других сингулярных точек. 
где $d \mu$ - индуцированная форма площади на поверхности. Он был введен Уиллмором в связи с вариационными задачами [84]. При этом Уиллмор был первым, кто сформулировал глобальную проблему конформной геометрии поверхностей - гипотезу Уиллмора, которую мы обсудим позднее. Уравнения Эйлера-Лагранжа для этого функционала имеют вид

$$
\Delta H+2 H\left(H^{2}-K\right)=0,
$$

где $\Delta$ - оператор Лапласа-Бельтрами на поверхности. Поверхности, удовлетворяющие этому уравнению, называются поверхностями Уиллмора.

Заметим, что $H=\frac{\varkappa_{1}+\varkappa_{2}}{2}$ и по теореме Гаусса-Бонне для компактной ориентированной поверхности $\Sigma$ без границы мы имеем

$$
\int_{\Sigma} K d \mu=\int_{\Sigma} \varkappa_{1} \varkappa_{2} d \mu=2 \pi \chi(\Sigma),
$$

где $\chi(\Sigma)$ - эйлерова характеристика $\Sigma$. Добавлением топологического члена к $\mathscr{W}$ мы получим функционал с теми же самыми экстремалями среди замкнутых поверхностей и можем упростить вариационную задачу. Это имеет место для сфер, когда рассматривая функционал

$$
\widehat{\mathscr{W}}(\Sigma)=\int\left(H^{2}-K\right) d \mu=\mathscr{W}(\Sigma)-2 \pi \chi(\Sigma),
$$

мы получаем, что ${ }^{10}$

$$
\widehat{\mathscr{W}}=\frac{1}{4} \int_{\Sigma}\left(\varkappa_{1}-\varkappa_{2}\right)^{2} d \mu .
$$

Напомним, что точка поверхности называется омбилической, если в ней $\varkappa_{1}=\varkappa_{2}$. Поверхность называется вполне омбилической, если все ее точки являются омбилическими. По теореме Хопфа вполне омбилическая поверхность в $\mathbb{R}^{3}$ является областью либо в круглой сфере, либо на плоскости. Это дает для сфер нижнюю оценку на функционал Уиллмора и описание всех его минимумов:

для сфер

$$
\mathscr{W}(\Sigma) \geqslant 4 \pi
$$

и $\mathscr{W}(\Sigma)=4 \pi$, если и только если $\Sigma-$ круглая сфера.

Для поверхностей большего рода этот трюк не работает.

Функционал $\widehat{\mathscr{W}}$ был введен Томсеном [85] и Бляшке [86], называвшими его конформной площадью по следующим причинам:

1) величина $\left(H^{2}-K\right) d \mu$ инвариантна относительно конформных преобразований объемлющего пространства и, следовательно, для компактной ориентированной поверхности $\Sigma \subset \mathbb{R}^{3}$ и конформного преобразования $G: \overline{\mathbb{R}}^{3} \rightarrow \overline{\mathbb{R}}^{3}$, переводящего $\Sigma$ в компактную поверхность, мы имеем

$$
\widehat{\mathscr{W}}(\Sigma)=\widehat{\mathscr{W}}(G(\Sigma))
$$

2) если $\Sigma$ - минимальная поверхность в $S^{3}$ и $\pi: S^{3} \rightarrow \overline{\mathbb{R}}^{3}$ - стереографическая проекция, отображающая $\Sigma$ в $\mathbb{R}^{3}$, то $\pi(\Sigma)$ - поверхность Уиллмора.

\footnotetext{
10Этот прием подобен инстантонному трюку, приведшему к открытию автодуальных связностей.
} 
Более того, как показано в [87],

3) вне омбилических точек определен дифференциал четвертой степени $\widehat{A}(d z)^{4}$, который голоморфен для поверхностей Уиллмора.

Мы излагаем эти результаты в приложении 2.

Согласно 2), существуют примеры компактных замкнутых поверхностей Уиллмора. Заметим, что не все поверхности Уиллмора являются стереографическими проекциями минимальных поверхностей в $S^{3}$ (впервые это было показано для торов в [88]).

Из 3) следует, что вне омбилических точек поверхности Уиллмора допускают хорошее описание, подобное описанию поверхностей постоянной средней кривизны в терминах голоморфности квадратичного дифференциала Хопфа. Но существуют примеры компактных поверхностей Уиллмора, которые содержат целые кривые, состоящие из омбилических точек [89].

Согласно 1), минимум функционала Уиллмора в каждом топологическом классе поверхностей конформно инвариантен и, следовательно, вырожден. Заметим, что существование минимума, который является вещественно-аналитической поверхностью, было доказано для торов Саймоном [90] и для поверхностей рода $g \geqslant 2$ Бауэром и Кувертом [91]. Недавно Шмидт представил доказательство следующего результата: для заданного рода и конформного класса ориентированной поверхности функционал Уиллмора достигает своего минимума на поверхности, которая а priori может иметь точки ветвления или быть разветвленным накрытием погруженной поверхности [92]. Его техника использует представление Вейерштрасса и некоторые идеи из [5]. ${ }^{11}$

Брайант инициировал программу классификации сфер Уиллмора, опираясь на то, что голоморфный дифференциал четвертой степени на сфере тождественно равен нулю и, следовательно, сферы Уиллмора допускают описание в терминах некоторых алгебро-геометрических данных [87]. Имеет место следующее утверждение:

образ минимальной поверхности в $\mathbb{R}^{3}$ при преобразовании Мёбиуса $\left(x-x_{0}\right) \rightarrow\left(x-x_{0}\right) /\left|x-x_{0}\right|^{2}$ является поверхностью Уиллмора, и каждая минимальная поверхность с плоскими концами переходит при преобразовании Мёбиуса с центром $x_{0}$ вне поверхности в такую гладкую компактную поверхность Уиллмора $\Sigma^{\prime}$, что

$$
\mathscr{W}\left(\Sigma^{\prime}\right)=4 \pi n,
$$

где $n$ - число плоских концов поверхности $\Sigma$.

Брайант доказал, что все сферы Уиллмора являются образами при преобразованиях Мёбиуса минимальных поверхностей с плоскими концами, что случай $n=1$ отвечает круглым сферам, что не существует таких сфер с $n=2$ и 3 , и описал все сферы Уиллмора с $n=4$. Позднее в [93] было доказано, что сферы Уиллмора существуют при всех четных $n \geqslant 6$ и всех нечетных $n \geqslant 9$. Оставшиеся случаи $n=5$ и 7 были окончательно исключены в [94].

Гипотеза Уиллмора утверждает, что для торов

$$
\mathscr{W} \geqslant 2 \pi^{2}
$$

и функционал Уиллмора достигает своего минимума на торе Клиффорда и его образах при конформных преобразованиях $\overline{\mathbb{R}}^{3}$.

\footnotetext{
${ }^{11}$ См. приложение 1.
} 
Тор Клиффорда был уже определен в п. 4.5.

Так как функционал Уиллмора конформно инвариантен и стереографическая проекция $\pi: S^{3} \rightarrow \overline{\mathbb{R}}^{3}$ конформна, мы не будем различать гипотезу Уиллмора для торов и ее версию для торов в $S^{3}$, в которой функционал Уиллмора заменяется на

$$
\mathscr{W}_{S^{3}}=\int\left(H^{2}+1\right) d \mu, \quad \mathscr{W}_{S^{3}}(\Sigma)=\mathscr{W}(\pi(\Sigma)) .
$$

Уиллмор сформулировал свою гипотезу в [84], где проверил ее для круглых торов вращения.

Она доказана во многих специальных случаях:

1) для трубчатых торов, получающихся таким проносом окружности вдоль замкнутой кривой, что центр окружности лежит на кривой, а сама окружность лежит в нормальной плоскости к кривой, доказательство дано Шиохамой и Такаги [95] и Уиллмором [96] (если разрешить радиусу окружности варьироваться, мы получим канальные торы, для которых гипотеза была доказана в [97]);

2) для торов вращения - Лангером и Зингером [98];

3) для торов, конформно эквивалентных $\mathbb{R}^{2} / \Gamma(a, b)$, где $0 \leqslant a \leqslant 1 / 2$, $\sqrt{1-a^{2}} \leqslant b \leqslant 1$ и решетка $\Gamma(a, b)$ порождена векторами $(1,0)$ и $(a, b)$ (Ли-Яо [99]);

4) предыдущий результат Ли и Яо был улучшен Монтьелем и Росом, распространившим его на замкнутую область $\left(a-\frac{1}{2}\right)^{2}+(b-1)^{2} \leqslant \frac{1}{4}[100]$;

$5)$ для торов в $S^{3}$, инвариантных относительно антиподального отображения (Рос [101]);

6) поскольку Ли и Яо также доказали [99], что если поверхность имеет точку самопересечения кратности $n$, то $\mathscr{W} \geqslant 4 \pi n$, гипотеза установлена для торов с самопересечениями.

Некоторые другие частные результаты были получены в [102], [103].

В статье [104] формула второй вариации функционала $\mathscr{W}$ для тора Клиффорда была вычислена и было доказано, что она неотрицательна. Формула второй вариации для общих поверхностей Уиллмора была получена в [105].

В общем случае гипотеза Уиллмора остается открытой.

Мы обсудим некоторый новый подход, примененный в [5], в следующем параграфе.

Согласно (53), следующая гипотеза является специальным случаем гипотезы Уиллмора, который тоже остается открытым:

для минимальных торов в $S^{3}$ объем ограничен снизу величиной $2 \pi^{2}$ и достигает своего минимума на торе Клиффорда в $S^{3}$.

Согласно теореме Ли-Яо о поверхностях с самопересечениями, эта гипотеза вытекает из следующей гипотезы Сяна-Лоусона:

тор Клиффорда является единственным минимальным тором, вложенным в $S^{3}$.

Поскольку голоморфный дифференциал четвертой степени на торе имеет постоянные коэффициенты, существуют две возможности: он равен нулю или $c(d z)^{4}, c=$ const $\neq 0$.

В первом случае тор получается как образ минимального тора с плоскими концами при отображении Мёбиуса. По очевидным причинам не существует торов с $n=1$ и 2 концами. Случай $n=3$ был исключен Куснером и 
Шмиттом, которые также построили примеры с $n=4$ [106]. Первые примеры минимальных прямоугольных торов с четырьмя плоскими концами были построены Костой [107]. Недавно Шамаев построил примеры таких торов для любого четного $n \geqslant 6$ [108]. Хотя из конструкции ясно, что эти торы не имеют точек ветвления, это было строго доказано только для $n=6,8$ и 10 .

Во втором случае уравнения типа Кодацци для торов Уиллмора без омбилических точек совпадают с четырехчастичной решеткой Тоды [109], [110]. ${ }^{12}$ Тэта-формулы для таких торов Уиллмора выведены в [110] с помощью функций Бейкера-Ахиезера, связанных с этой решеткой Тоды.

Другая конструкция торов Уиллмора методами теории интегрируемых систем предложена в [111].

Для поверхностей большего рода кандидаты на минимумы для функционала Уиллмора были предложены Куснером [112].

Существует гипотеза, что для торов в $\mathbb{R}^{4}$ функционал Уиллмора $\int|H|^{2} d \mu$ достигает своего минимума на торе Клиффорда в $\mathbb{R}^{4}$, т.е. на произведении окружностей одинакового радиуса (см. [113], [114]). Так как этот тор лагранжев, то последняя гипотеза ослабляется следующим образом: тор Клиффорда является минимумом функционала Уиллмора на меньшем классе лагранжевых торов. Она обсуждается в [115], где доказано, что $\mathscr{W}$ достигает своего минимума среди лагранжевых торов на некотором вещественно-аналитическом торе.

Мы не обсуждаем обобщения функционала Уиллмора для поверхностей в произвольных римановых многообразиях:

$$
\int\left(|H|^{2}+\widehat{K}\right) d \mu
$$

где $\widehat{K}$ - секционная кривизна объемлющего пространства вдоль касательной плоскости к поверхности. Величина $\left(|H|^{2}-K+\widehat{K}\right) d \mu$ инвариантна относительно конформных преобразований объемлющего пространства [116].

В статье [11] предложено иное обобщение функционала Уиллмора для поверхностей в трехмерных группах Ли. Оно основано на спектральной теории операторов Дирака, входящих в представления Вейерштрасса (см. п. 5.5).

Мы также должны упомянуть поток Уиллмора, который сходен потоку средней кривизны и уменьшает значение функционала $\mathscr{W}$ (см. статью [117] и ссылки в ней).

Мы закончим эту часть замечанием об условно уиллморовских поверхностях, которые по определению являются критическими точками функционала Уиллмора, ограниченного на пространство поверхностей того же самого конформного типа. Лангером впервые было отмечено, что компактные поверхности постоянной средней кривизны в $\mathbb{R}^{3}$ являются условно уиллморовскими, поскольку для них отображение Гаусса является гармоническим [118]. За основами теории таких поверхностей мы отсылаем к [119].

5.2. Спектральные кривые и гипотеза Уиллмора. Как показано в [1], в терминах потенциала $U$ представления Вейерштрасса тора в $\mathbb{R}^{3}$ функционал Уиллмора принимает вид

$$
\mathscr{W}=4 \int_{M} U^{2} d x d y
$$

\footnotetext{
${ }^{12}$ См. приложение 2.
} 
Таким образом он измеряет возмущение свободного оператора.

Мы напомним, что гипотеза Уиллмора утверждает, что этот функционал для торов достигает своего минимума на торе Клиффорда, для которого функционал равен $2 \pi^{2}$.

Исходя из наблюдения, что функционал Уиллмора является первым интегралом мВН потока, деформирующего торы в торы с сохранением конформного класса (см. п. 3.1), мы выдвинули в 1995 году следующую гипотезу (см. [1]): нестационарный (по отношению к мВН-потоку) тор не может быть локальным минимумом функционала Уиллмора.

Она базировалась на предположении, что минимум такой вариационной задачи не должен быть вырожден и поэтому должен быть стабилен по отношению к солитонным деформациям, заданными уравнениями из иерархии мВН и сохраняющими значение функционала Уиллмора. Из теории солитонов известно, что эти уравнения линеаризуются на многообразии Якоби нормализованной спектральной кривой и, в общем случае, эти линейные потоки порождают все многообразие Якоби, которое является абелевым многообразием комплексной размерности $p_{g}(\Gamma)$, или подмногообразие Прима многообразия Якоби.

Геометрический аналог этой гипотезы был сформулирован в [3], где мы ввели понятие спектрального рода тора как $p_{g}(\Gamma)$ :

для заданного конформного класса торов в $\mathbb{R}^{3}$ минимумы функци-

онала Уиллмора достигаются на торах с наименьшим спектральным родом.

В [3] мы предложили следующее объяснение нижним оценкам для $\mathscr{W}:$ для малых возмущений нулевого потенциала $U=0$ представление Вейерштрасса дает плоскости, которые не свертываются в торы, и, поскольку для поверхностей в $\mathbb{R}^{3}$ функционал Уиллмора - это квадрат $L_{2}$-нормы $U$, нижняя оценка показывает, каким большим должно быть возмущение нулевого потенциала, чтобы заставить плоскости свернуться в торы.

Стратегия доказательства гипотезы Уиллмора после доказательства последней гипотезы состоит в вычислении значений функционала Уиллмора для торов минимального спектрального рода (с помощью формулы (44) или иным способом) и проверке гипотезы Уиллмора.

Мы уже упомянули в тексте статью Шмидта [5]. Эта статья содержит ряд интересных результатов. ${ }^{13}$ Для наших целей мы излагаем только результаты, касающиеся асимптотического поведения спектральной кривой. Хотя до настоящего времени мы не разобрали все детали в [5], мы должны сказать, что

в действительности, статъя [5] предлагает доказательство только нашей последней гипотезы (см. выше); значение $p_{a}\left(\Gamma_{\psi}\right)$ a priori неограничено, но в [5] вычисления функционала Уиллмора проведены только в случае минимально возможных значений $u p_{g}(\Gamma), u p_{a}\left(\Gamma_{\psi}\right)$.

Следуя духу предыдущих гипотез, естественно предположить, что

\footnotetext{
${ }^{13}$ В этой статье также предложено доказательство того, что спектральный род условно уиллморовского тора в $\mathbb{R}^{3}$ конечен. Другое доказательство этого факта было предложено Кричевером (неопубликовано). Это факт нетривиален даже для уиллморовских торов, поскольку трюк, использующий теорию солитонов и применявшийся в [62], [77] к гармоническим торам в $S^{3}$ и торам постоянной средней кривизны в $\mathbb{R}^{3}$ (см. также в [79]), в данной ситуации работает не для всех торов. Он применим только к тем торам, которые описываются четырехчастичной решеткой Тоды и не содержат омбилических точек, в которых $e^{\beta}=0$ (см. приложение 2).
} 
для заданных конформного класса торов в $\mathbb{R}^{3}$ и спектрального рода минимумы функционала Уиллмора достигаются на торах с наименьшим значением $p_{a}\left(\Gamma_{\psi}\right)$.

Эта гипотеза тоже согласована с солитонным подходом, поскольку дополнительные степени свободы, отвечающие разности $p_{a}(\Gamma)-p_{g}(\Gamma)$ (или части ее, если потоки линеаризуются на многообразии Прима), также отвечают солитонным деформациям.

По нашему мнению эти гипотезы интересны сами по себе. Заметим, что доказательства последних двух из них вместе с вычислениями значений функционала Уиллмора при минимально возможных значениях $p_{g}(\Gamma)$ и $p_{a}\left(\Gamma_{\psi}\right)$ приведут к проверке гипотезы Уиллмора.

Мы также хотим отметить другую интересную задачу: как обобщить эту теорию спектральных кривых для компактных погруженных поверхностей больших родов?

5.3. Нижние оценки для функционала Уиллмора. В [6] в некотором специальном случае мы установили нижнюю оценку для функционала Уиллмора, квадратичную по размерности ядра оператора Дирака.

Представим сферу как бесконечный цилиндр $Z$, компактифицированный парой точек так, что $z=x+i y-$ конформный параметр на $Z$, переменная $y$ определена по модулю $2 \pi, x \in \mathbb{R}$ и эти две бесконечно удаленные точки получаются в пределах $x \rightarrow \pm \infty$.

Лемма 3 [6]. Для сферы в $\mathbb{R}^{3}$ функиия $\psi$ и потенииал U имеют следующие асимптотики:

$\left|\psi_{1}\right|^{2}+\left|\psi_{2}\right|^{2}=C_{ \pm} e^{-|x|}+O\left(e^{-2|x|}\right), \quad U=U_{ \pm} e^{-|x|}+O\left(e^{-2|x|}\right) \quad$ при $\quad x \rightarrow \pm \infty$,

где $C_{ \pm}$и $U_{ \pm}-$постоянные. Если $C_{+}=0$ или $C_{-}=0$, то соответствующая отмеченная точка $x=+\infty$ или $x=-\infty$ является точкой ветвления.

Ядро оператора $\mathscr{D}$ на сфере образовано такими решениями $\psi$ уравнения $\mathscr{D} \psi=0$ на иилиндре, что $\left|\psi_{1}\right|^{2}+\left|\psi_{2}\right|^{2}=O\left(e^{-|x|}\right)$ при $x \rightarrow \pm \infty$.

Предположим, что потенциал $U$ оператора Дирака зависит только от $x$. Например, такая ситуация имеет место для сферы вращения и при этом $y$ - это угол вращения. Но это имеет место не только для сфер вращения, а и для многих других поверхностей с внутренней $S^{1}$-симметрией, отражающейся в представлении Вейерштрасса.

Tеорема 13 [6]. Пусть $\mathscr{D}$ - оператор Дирака на сфере $M=S^{2}$ с вещественным потенциалом $U=V$, зависящим только от $x$. Тогда

$$
\int_{M} U^{2} d x \wedge d y \geqslant \pi N^{2}
$$

где $N=\operatorname{dim}_{\mathbb{H}} \operatorname{Ker} \mathscr{D}=\frac{1}{2} \operatorname{dim}_{\mathbb{C}} \operatorname{Ker} \mathscr{D} . Э_{\text {м }}$ минимумы достигаются на потенuиалах

$$
U_{N}(x)=\frac{N}{2 \cosh x} .
$$

Доказательство этой теоремы основано на методе обратной задачи рассеяния, примененном к одномерному оператору Дирака. Эта квадратичная оценка возникает из формул следов Фаддеева и Тахтаджяна [120].

Вместе с доказательством теоремы 13 мы высказали следующую гипотезу. 
ГиПотезА 1 [6]. Оценка (54) верна для всех операторов Дирака на двумерной сфере.

Прежде чем дать доказательство теоремы 13, мы изложим одно из следствий данной гипотезы - теорему 14.

Очень скоро после электронной публикации работы [6] Фридрих указал следующее следствие этой гипотезы. ${ }^{14}$

Теорема 14 [121]. Предположим, что гипотеза 1 верна. Пусть $\lambda$ - собственное значение оператора Дирака на двумерном спинорном многообразии, гомеоморфном двумерной сбере $S^{2}$. Имеет место неравенство

$$
\lambda^{2} \operatorname{Area}(M) \geqslant \pi m^{2}(\lambda),
$$

где $m(\lambda)$ - кратность собственного значения $\lambda$.

Заметим, что, согласно симметрии (4) оператора $\operatorname{Ker} D$, кратность собственного значения всегда четна. Для случая $m(\lambda)=2$ неравенство (55) было доказано Бэром [122].

ДокАЗАТЕЛЬСтво теоремы 14. Сначала напомним определение оператора Дирака на спинорном многообразии (см. подробные изложения в [123], [124]).

Спинорное $n$-мерное многообразие $M$ - это риманово многообразие со спинорным расслоением $E$ над $M$ таким, что в каждой точке $p \in M$ определено умножение Клиффорда

$$
T_{p} M \times E_{p} \rightarrow E_{p}
$$

и при этом

$$
v \cdot w \cdot \varphi+w \cdot v \cdot \varphi=-2(v, w) \varphi, \quad v, w \in T_{p} M, \quad \psi \in E_{p} .
$$

Мы также предполагаем, что задана риманова связность $\nabla$, индуцирующая связность на $E$. Тогда в каждой точке $p \in M$ оператор Дирака определяется формулой

$$
D \varphi=\sum_{k=1}^{n} e_{k} \cdot \nabla_{e_{k}} \varphi
$$

где $e_{1}, \ldots, e_{n}$ - ортонормированный базис в $T_{p} M$ и $\varphi$ - сечение расслоения $E$.

Рассмотрим, для примера, двумерное спинорное многообразие $M$ с плоской метрикой. Алгебра Клиффорда $\mathcal{C} l_{2}$ изоморфна $\mathbb{H}$. Таким образом, мы имеем спинорное $\mathbb{C}^{2}$-расслоение над $M$ (здесь мы отождествляем $\mathbb{H}$ с $\mathbb{C} \oplus \mathbb{C}$ ). Для плоской метрики на $M$ умножение Клиффорда задается матрицами

$$
e_{1}=e_{x}=\left(\begin{array}{cc}
0 & 1 \\
-1 & 0
\end{array}\right), \quad e_{2}=e_{y}=\left(\begin{array}{cc}
0 & -i \\
-i & 0
\end{array}\right)
$$

Легко проверить, что

$$
e_{x} e_{y}+e_{y} e_{x}=0, \quad e_{x}^{2}=e_{y}^{2}=-1
$$

\footnotetext{
14Эта гипотеза была окончательно доказана Ферусом, Лешке, Педитом и Пинкаллем в [7] вместе с обобщением (54), так называемой формулой Плюккера, для поверхностей большего рода, для всех $g \geqslant 0$ (мы излагаем это в п. 5.4).
} 
Оператор Дирака $D_{0}$ задается формулой

$$
D_{0}=e_{x} \cdot \partial_{x}+e_{y} \cdot \partial_{y}=2\left(\begin{array}{cc}
0 & \partial \\
-\bar{\partial} & 0
\end{array}\right)=2 \mathscr{D}_{0},
$$

и его квадрат равен оператору Лапласа (с точностью до знака):

$$
D_{0}^{2}=-\partial_{x}^{2}-\partial_{y}^{2}
$$

Для конформно-евклидовой метрики $e^{\sigma} d z d \bar{z}$ оператор Дирака имеет вид

$$
D=e^{-3 \sigma / 4} D_{0} e^{\sigma / 4}
$$

(см. [125]). Следовательно, задача на собственные значения

$$
D \varphi=\lambda \varphi
$$

для оператора Дирака, ассоциированного с этой метрикой, принимает вид

$$
D_{0}\left[e^{\sigma / 4} \varphi\right]-\lambda e^{\sigma / 2}\left[e^{\sigma / 4} \varphi\right]=0
$$

что переписывается как

$$
\left(\mathscr{D}_{0}+U\right) \psi=0, \quad U=-\frac{\lambda e^{\sigma / 2}}{2}, \quad \psi=e^{\sigma / 4} \varphi .
$$

Если гипотеза 1 верна, то мы имеем неравенство

$$
\int_{M} U^{2} d x \wedge d y=\frac{\lambda^{2}}{4} \operatorname{Area}(M) \geqslant \pi\left(\frac{\operatorname{dim}_{\mathbb{C}} \operatorname{Ker}\left(D_{0}+U\right)}{2}\right)^{2}=\pi \frac{m^{2}(\lambda)}{4} .
$$

Теорема 14 доказана.

ДоКАЗАТЕЛЬСТВо ТЕОРЕМЫ 13 . Если потенциал $U$ зависит только от $x$, то линейное пространство решений уравнения $\mathscr{D} \psi=0$ на сфере $S^{2}=Z \cup \pm \infty=$ $\mathbb{R}_{x} \times S_{y}^{1} \cup \infty$ порождено такими функциями вида $\psi(x, y)=\varphi(x) e^{\varkappa y}$, что

$$
L \varphi:=\left[\left(\begin{array}{cc}
0 & \partial_{x} \\
-\partial_{x} & 0
\end{array}\right)+\left(\begin{array}{cc}
2 U & 0 \\
0 & 2 U
\end{array}\right)\right] \varphi=\left(\begin{array}{cc}
0 & i \varkappa \\
i \varkappa & 0
\end{array}\right) \varphi
$$

где $e^{2 \pi \varkappa}=-1$ (это условие определяет спинорное расслоение над сферой, см. [45]) и $\varphi$ экспоненциально убывает при $x \rightarrow \pm \infty$. Последнее означает, что $\varphi$ является связанным состоянием оператора $L$, т.е. $\varkappa$ принадлежит дискретному спектру, инвариантному относительно комплексного сопряжения $\varkappa \rightarrow \bar{\varkappa}$. Следовательно, $\operatorname{dim}_{\mathbb{C}} \mathscr{D}=2 N$ - это удвоенное число связанных состояний, удовлетворяющих условию $\operatorname{Im} \varkappa>0$.

Формула следов (76) (см. приложение 3) для случая $p=q=2 U$ примет вид

$$
\int_{-\infty}^{\infty} U^{2}(x) d x=-\frac{1}{4 \pi} \int_{-\infty}^{\infty} \log \left(1-|b(k)|^{2}\right) d k+\sum_{j=1}^{N} \operatorname{Im} \varkappa_{j}
$$

Для заданного $\operatorname{dim} \operatorname{Ker} \mathscr{D}=N$ функционал $\int_{M} U^{2}(x) d x \wedge d y=2 \pi \int_{-\infty}^{\infty} U^{2}(x) d x$ достигает своего минимума на потенциале со следующими спектральными данными:

$$
b(k) \equiv 0, \quad \varkappa_{k}=\frac{i(2 k-1)}{2}, \quad k=1, \ldots, N,
$$


и мы имеем

$$
\int_{S^{2}} U^{2}(x) d x \wedge d y \geqslant 2 \pi\left(\frac{1}{2}+\frac{3}{2}+\cdots+\frac{N}{2}\right)=\pi N^{2} .
$$

В действительности, существует $N$-мерное семейство потенциалов, параметризованное $\lambda_{1}, \ldots, \lambda_{N}$, и, более того, это семейство инвариантно относительно уравнений мКдФ. Легко показать, что каждое такое семейство содержит потенциал $U_{N}=N /(2 \cosh x) ; p_{N}(x)=2 U_{N}(x)=N / \cosh x-$ это знаменитый $N$-солитонный потенциал оператора Дирака.

Теорема 13 доказана.

Мы видим, что равенство в (54) достигается на некоторых специальных сфеpax, которые являются частным случаем так называемых солитонных сфер. По определению это - сферы, для которых потенциал оператора Дирака $\mathscr{D}$ является солитонным (безотражательным) потенциалом $U(x)$. Разумно выделить специальный подкласс солитонных сфер, заданный условием, что все полюсы $\varkappa_{1}, \ldots, \varkappa_{N}, \operatorname{Im} \varkappa_{k}>0$, коэффициента прохождения $T(k)$ имеют вид $\frac{(2 m+1) i}{2}$, $m \in \mathbb{N}$.

Солитонные сферы легко строятся по спектральным данным с помощью метода обратной задачи рассеяния (см. (77) в приложении 3).

Мы показали в [6], что

а) нижняя оценка (54) достигает равенства на солитонных сферах, отвечающих потенциалам $U_{N}=\frac{N}{2 \cosh x}$;

б) в общем случае солитонная сфера не является сферой вращения; ${ }^{15}$

в) класс солитонных сфер сохраняется мКдФ-деформациями (заметим, что они задаются $(1+1)$-уравнениями), для которых интегралы КрускалаМиуры являются интегралами движения;

г) солитонные сферы, отвечающие потенциалам $U_{N}=\frac{N}{2 \cosh x}$, описываются в терминах рациональных функций, ${ }^{16}$ т.е. могут быть названы рациональными сферами;

д) солитонные сферы, для которых каждый полюс $\varkappa_{j}$ имеет вид $\frac{\left(2 m_{j}+1\right) i}{2}$, являются критическими точками функционала Уиллмора, ограниченного на класс сфер с одномерными потенциалами.

5.4. Формула Плюккера. Наши попытки доказать гипотезу 1 не удались из-за отсутствия хорошо развитого метода обратной задачи рассеяния для двумерных операторов. Но в замечательной работе [7] эта гипотеза была доказана вместе с ее обобщением на поверхности произвольного рода методами алгебраической геометрии.

Как отмечено в [8], следующее утверждение может быть выведено из результатов статьи [126] (см. также [127]).

15 Действительно, обозначим через $f_{1}=\varphi_{1}(x) e^{\varkappa_{1} y}, \ldots, f_{n}=\varphi_{N} e^{\varkappa_{N} y}$ различные образующие $\operatorname{Ker} \mathscr{D}$. Тогда любая линейная комбинация $f=\alpha_{1} f_{1}+\cdots+\alpha_{N} f_{N}$ посредством представления Вейерштрасса задает сферу в $\mathbb{R}^{3}$. Если существует пара не равных нулю коэффициентов $\alpha_{j}$ и $\alpha_{k}$ таких, что $\operatorname{Im} \varkappa_{j} \neq \operatorname{Im} \varkappa_{k}$, то сфера не является поверхностью вращения.

${ }^{16}$ Из формул восстановления (77) ясно, что это верно для всех безотражательных потенциалов. 
ПРЕДЛОЖЕНИЕ 15. Пусть $E-C^{2}$-расслоение над поверхностью $M, u$ пусть $\psi$ - нетривиальное сечение $E$ такое, что $\mathscr{D} \psi=0$. Тогда нули $\psi$ изолированы и для любой локальной координаты $z$ на $M$ с иентром в нуле $p$ функции $\psi: \psi(p)=0, z(p)=0$, мы имеем

$$
\psi=z^{k} \varphi+O\left(|z|^{k+1}\right),
$$

где $\varphi$ - локальное сечение $E$, которое не обращается в нуль в окрестности $р$. Целое число $k$ корректно определено и не зависит от выбора координаты $z$.

Целое число $k$ называется порядком нуля $p$ :

$$
\operatorname{ord}_{p} \psi=k \text {. }
$$

Теперь напомним уравнение (см. предложение 1 в п. 2.1):

$$
\alpha_{z \bar{z}}+U^{2}-|A|^{2} e^{-2 \alpha}=0, \quad e^{\alpha}=\left|\psi_{1}\right|^{2}+\left|\psi_{2}\right|^{2} .
$$

Для простоты предположим, что $M$ - сфера и $E$ - спинорное расслоение. Если сечение $\psi$ спинорного расслоения нигде не равно нулю, то оно задает поверхность в $\mathbb{R}^{3}$ и, интегрируя левую часть (56) по $M$, мы получаем

$$
\int_{M} \alpha_{z \bar{z}} d x \wedge d y+\int_{M} U^{2} d x \wedge d y-\int_{M}|A|^{2} e^{-2 \alpha} d x \wedge d y=0 .
$$

По теореме Гаусса первый член равен

$$
-\frac{1}{4} \int_{M}\left(-4 \alpha_{z \bar{z}} e^{-2 \alpha}\right) e^{2 \alpha} d x \wedge d y=-\frac{1}{4} \int_{M} K d \mu=-\pi,
$$

где $K$ - гауссова кривизна и $d \mu$ - мера, отвечающая индуцированной метрике. Таким образом, ${ }^{17}$

$$
\int_{M} U^{2} d x \wedge d y=\pi+\int_{M}|A|^{2} e^{-2 \alpha} d x \wedge d y \geqslant-\int_{M} \alpha_{z \bar{z}} d x \wedge d y=\pi .
$$

В общем случае для каждой поверхности и каждого сечения $\psi$, удовлетворяющего уравнению $\mathscr{D} \psi=0$ (т.е. мы не предполагаем, что $\psi$ нигде не равно нулю), мы имеем

$$
\begin{aligned}
\int_{M} U^{2} d x \wedge d y & =\pi\left(-\operatorname{deg} E_{0}+\sum_{p} \operatorname{ord}_{p} \psi\right)+\int_{M}|A|^{2} e^{-2 \alpha} d x \wedge d y \\
& \geqslant \pi\left(-\operatorname{deg} E_{0}+\sum_{p} \operatorname{ord}_{p} \psi\right)
\end{aligned}
$$

(см. [8]). Подынтегральное выражение $|A|^{2} e^{-2 \alpha}$ имеет особенности в нулях $\psi$, но интеграл сходится и неотрицателен.

Возвращаясь к случаю спинорных расслоений над сферами (deg $E_{0}=$ $g-1=-1)$ и предполагая, что $\operatorname{dim}_{\mathbb{H}} \operatorname{Ker} \mathscr{D}=N$, мы выберем точку $p$ и

\footnotetext{
17 Для общих комплексных кватернионных расслоений $L=E_{0} \oplus E_{0}$ мы имеем $\int_{M} \alpha_{z \bar{z}} d x \wedge$ $d y=\pi \operatorname{deg} E_{0}=\pi d$.
} 
функцию $\psi \in \operatorname{Ker} \mathscr{D}$ так, что $\operatorname{ord}_{p} \psi=\operatorname{dim} \operatorname{Ker}_{\mathbb{H}} \mathscr{D}-1=N-1$. Теперь подставим $\psi$ в $(56)$ и получим

$$
\int_{M} U^{2} d x \wedge d y=\pi(1+N-1)+\int_{M}|A|^{2} e^{-2 \alpha} d x \wedge d y \geqslant \pi N .
$$

Но эта оценка очень грубая, поскольку мы видим из доказательства теоремы 13, что не только функция из Ker $\mathscr{D}$ с максимальным порядком нулей вносит свой вклад в нижние оценки для функционала Уиллмора и необходимо рассматривать флаг функций.

В [7] была открыта глубокая аналогия между этой задачей и соотношениями Плюккера, которые связывают степени и индексы ветвления кривых, ассоциированных с некоторой алгебраической кривой в $\mathbb{C} P^{n}$. Это позволило выписать такой флаг и учесть вклад всего ядра оператора $\mathscr{D}$ в функционал Уиллмора. В итоге это привело к установлению нижних оценок для функционала Уиллмора, которые квадратичны по $\operatorname{dim}_{\mathbb{H}} \operatorname{Ker} \mathscr{D}$.

Чтобы сформулировать главный результат работы [7], мы введем некоторые определения. Пусть $H$ - подпространство в $\operatorname{Ker} \mathscr{D}$. Для каждой точки $p$ мы положим

$$
n_{0}(p)=\min _{\operatorname{ord}} \psi \quad \text { при } \quad \psi \in H .
$$

Затем последовательно определим

$$
n_{k}(p)=\min \operatorname{ord}_{p} \psi \quad \text { при } \psi \in H \text { таких, что } \operatorname{ord}_{p} \psi>n_{k-1}(p) .
$$

Мы имеем последовательность пробелов Вейерштрасса:

$$
n_{0}(p)<n_{1}(p)<\cdots<n_{N-1}(p), \quad N=\operatorname{dim}_{\mathbb{H}} H,
$$

и цепочку вложений

$$
H=H_{0} \supset H_{1} \supset \cdots \supset H_{N-1},
$$

где $H_{k}$ состоит из таких $\psi$, что $\operatorname{ord}_{p} \psi \geqslant n_{k}(p)$. Теперь мы определим порядок линейной системы $H$ в точке $p$ как

$$
\operatorname{ord}_{p} H=\sum_{k=0}^{N-1}\left(n_{k}(p)-k\right)=\sum_{k=0}^{N-1} n_{k}(p)-\frac{1}{2} N(N-1) .
$$

Мы говорим, что $p$ - точка Вейерштрасса, если $\operatorname{ord}_{p} H \neq 0$.

Теперь мы можем сформулировать основной результат этой теории.

TeOpema 15 [7]. Пустъ $H \subset \operatorname{Ker} \mathscr{D} u \operatorname{dim}_{\mathbb{H}} H=N$. Тогда

$$
\int_{M}|U|^{2} d x \wedge d y=\pi\left(N^{2}(1-g)+\operatorname{ord} H\right)+\mathscr{A}(M),
$$

где член $\mathscr{A}(M)$ неотрицателен и сводится $\kappa \int_{M}|A| e^{-2 \alpha} d x \wedge d y$ в случае (57).

В действительности основной результат из [7] верен для операторов Дирака с комплексно сопряженными потенциалами $U=\bar{V}^{18}$ на произвольных комплексных кватернионных линейных расслоениях произвольной степени $d$ (это

\footnotetext{
${ }^{18} \mathrm{~B}$ этом случае $\frac{1}{4} \mathscr{W}=\int U V d x \wedge d y=\int|U|^{2} d x \wedge d y$, где $\mathscr{W}-$ функционал Уиллмора.
} 
прямо видно из доказательства) и объясняет выражение $\mathscr{A}(M)$ в терминах двойственных кривых:

$$
\int_{M}|U|^{2} d x \wedge d y=\pi(N((N-1)(1-g)-d)+\operatorname{ord} H)+\mathscr{A}(M),
$$

где $g-$ род поверхности $M$ и $d=\operatorname{deg} L=\operatorname{deg} E_{0}$. В теореме 15 мы полагаем $d=1-g$, т.е. рассматриваем случай, представляющий интерес для теории поверхностей.

Для $U=0$ мы имеем $\mathscr{A}(M)=0$ и формула Плюккера (58) сводится к исходному соотношению Плюккера для алгебраических кривых (см., например, [128]):

$$
\text { ord } H=N((N-1)(g-1)+d) .
$$

При $g=0$ мы имеем

СледСтвие 4 [7]. Гипотеза 1 верна: $\int U^{2} d x \wedge d y \geqslant \pi N^{2}$.

При $g \geqslant 1$ мы имеем эффективную нижнюю оценку только в терминах ord $H$, член, квадратичный по $N$, равен нулю при $g=1$ и отрицателен при $g>1$.

Для получения эффективных нижних оценок функционала Уиллмора в [7] было предложено использовать специальные линейные системы $H$. Пусть $\operatorname{dim} \operatorname{Ker} \mathscr{D}=N$. Возьмем в $\operatorname{Ker} \mathscr{D} k$-мерную линейную систему $H$, выделенную условием, что для всех $\psi \in H$ мы имеем $\operatorname{ord}_{p} \psi \geqslant N-k$ для некоторой заданной точки $p$. Последовательность пробелов Вейерштрасса удовлетворяет неравенству

$$
n_{l}(p) \geqslant N-k+l, \quad l=0,1, \ldots, k-1,
$$

и, следовательно, $\operatorname{ord}_{p} H \geqslant k(N-k)$. Из (58) мы получаем

$$
\int U^{2} d x \wedge d y \geqslant \pi\left(k^{2}(1-g)+k(N-k)\right)=k N-k^{2} g .
$$

Если $g=0$, то правая часть достигает своего максимума при $k=N$ и мы получаем оценку (54).

При $g \geqslant 1$ функция $f(x)=x N-x^{2} g$ достигает своего максимума в точке $x_{\max }=N /(2 g)$. Следовательно, правая часть в (59) достигает своего максимума либо при $k=[N /(2 g)]$, либо при $[N /(2 g)]+1$, т.е. в целой точке, наиболее близкой к $x_{\max }$. Из этого легко вывести грубую оценку, верную для всех $g$. Конечно, в специальных случаях эта оценка может быть улучшена, как, например, в случае $g=1$ :

СлеДСтвиЕ 5 [7]. Имеют место неравенства

$$
\int U^{2} d x \wedge d y \geqslant \frac{\pi}{4 g}\left(N^{2}-g^{2}\right)
$$

npu $g>1 u$

$$
\int U^{2} d x \wedge d y \geqslant \begin{cases}\frac{\pi N^{2}}{4} & \text { при } N \text { четном, } \\ \frac{\pi\left(N^{2}-1\right)}{4} & \text { при } N \text { нечетном }\end{cases}
$$

npu $g=1$. 
Доказательство теоремы 14 прямо проходит для вывода следующего утверждения.

СлЕДСТВИЕ 6 [7]. Для данного собственного значения $\lambda$ оператора Дирака на двумерном спинорном многообразии рода $g$ имеют место следующие неравенства

$$
\lambda^{2} \operatorname{Area}(M) \geqslant \begin{cases}\pi m^{2}(\lambda) & \text { при } g=0, \\ \frac{\pi}{g}\left(m^{2}(\lambda)-g^{2}\right) & \text { при } g \geqslant 1,\end{cases}
$$

где $m(\lambda)$ - кратность собственного значения $\lambda$.

Другое важное приложение формулы (61) касается нижних оценок для площади торов постоянной средней кривизны в $\mathbb{R}^{3}$ и минимальных торов в $S^{3}$. Из явной конструкции спектральных кривых (см. п. 4.6) видно, что в обоих случаях нормализованные спектральные кривые - это гиперэллиптические кривые

$$
\mu^{2}=P(\lambda)
$$

такие, что пара точек ветвления отвечает "бесконечностям" $\infty_{ \pm}$. Имеется еще $2 g$ других точек ветвления (здесь $g$ - род этой гиперэллиптической кривой), в которых мультипликаторы функций Флоке равны \pm 1 (по построению спектральной кривой). Более того, существует еще пара точек, переставляющихся гиперэллиптической инволюцией, в которых мультипликаторы тоже равны \pm 1 (торы строятся в терминах этих функций Флоке, как показано в [62] и [78]). Таким образом, мы имеем пространство $F$ с $\operatorname{dim}_{\mathbb{C}} F=2 g+2$, состоящее из решений уравнения $\mathscr{D} \psi=0$ с мультипликаторами \pm 1 . Возьмем 4 -листное накрытие $\widehat{M}$ тора $M$, которое удваивает периоды. Подъемы функций из $F$ на это накрытие являются двояко-периодическими функциями, т.е. являются сечениями одного и того же спинорного расслоения над $\widehat{M}$. Комплексная размерность ядра оператора $\mathscr{D}$, действующего на этом спинорном расслоении, по меньшей мере $2 g+2$, и поэтому $\operatorname{dim}_{\mathbb{H}} \operatorname{Ker} \mathscr{D} \geqslant g+1$. Применяя (61), мы получаем нижние оценки на $\int|U|^{2} d x \wedge d y$. Для торов постоянной средней кривизны мы имеем $H=1$ и $U=\frac{e^{\alpha}}{2}$. Следовательно,

$$
\int_{\widehat{M}} U^{2} d x \wedge d y=\frac{1}{4} \operatorname{Area}(\widehat{M})=\operatorname{Area}(M) .
$$

Для минимальных торов в $S^{3}$ мы имеем $U=-\frac{i e^{\alpha}}{2}$ и $\int_{\widehat{M}}|U|^{2} d x \wedge d y=\operatorname{Area}(M)$. Мы выводим следующее утверждение.

СЛЕДСТВИЕ 7. Для минимальных торов в $S^{3}$ и торов постоянной средней $к р и в и з н ы ~ в ~ \mathbb{R}^{3}$ спектрального рода g выполняются следующие нижние оценки на площадъ:

$$
\text { Area } \geqslant \begin{cases}\frac{\pi(g+1)^{2}}{4} & \text { при } g \text { нечетном, } \\ \frac{\pi\left((g+1)^{2}-1\right)}{4} & \text { при } g \text { четном. }\end{cases}
$$


В [7] отмечается, что из [62] следует, что для минимальных торов в $S^{3}$ оценка может быть улучшена заменой $g+1$ на $g+2$. Более того, она верна для энергии всех гармонических торов в $S^{3}$.

Недавно род спектральной кривой был применен Хаскинсом совершенно в другой задаче: при изучении специальных лагранжевых $T^{2}$-конусов в $\mathbb{C}^{3}[129]$. Им были получены линейные (по роду) нижние оценки для ряда величин, характеризующих геометрическую сложность таких конусов, и высказано предположение, что эти оценки можно улучшить до квадратичных. Отметим, что методы работы [129] совершенно отличны от методов, примененных в [7].

Вклад члена ord $H$ легко демонстрируется на примере солитонных сфер, у которых полюсы коэффициента прохождения имеют вид $(2 l+1) i / 2$. В этом случае ord Ker $\mathscr{D}$ вычисляет пробелы в заполнении этих энергетических уровней.

Недавно определение солитонных сфер было обобщено в духе нижних оценок для функционала Уиллмора: сфера называется солитонной, если для нее "неравенство Плюккера"

$$
\int_{M}|U|^{2} d x \wedge d y \geqslant \pi\left(N^{2}(1-g)+\operatorname{ord} H\right)
$$

становится равенством, т.е. $\mathscr{A}(M)=0[130]$.

Как было показано Боле и Петерсом [131], этот класс сфер содержит много других интересных примеров.

Прежде чем сформулировать их результат, мы напомним, что поверхности Брайанта - это, в точности, поверхности постоянной кривизны 1 в гиперболическом трехмерном пространстве [132]. Согласно [131], поверхность Брайанта $M$ в трехмерном шаре - модели Пуанкаре гиперболического пространства $B^{3} \subset \mathbb{R}^{3}$ есть гладкий брайантовский конец, если существует такая точка $p_{\infty}$ на асимптотической границе шара $\partial B^{3}$, что $M \cup p_{\infty}-$ конформно погруженный диск в $\mathbb{R}^{3}$. В общем случае поверхность Брайанта называется компактной поверхностью Брайанта с гладкими концами, если она конформно эквивалентна компактной поверхности с конечным числом выколотых точек, каждая из которых имеет окрестность, изометричную гладкому брайантовскому концу.

Это, очевидно, - обобщение минимальных поверхностей с плоскими концами.

Мы имеем следующую теорему.

Теорема 16 [131]. Сферы Брайанта с гладкими концами являются солитонными сферами. Возможные значения функционала Уиллмора для таких сфер - это $4 \pi N$, где $N$ - положительное натуральное число, отличное от 2 , 3,5 u 7 .

Как отмечено Боле и Петерсом, к этой теореме их привело наблюдение, что простейшие солитонные сферы, отвечающие потенциалам $U_{N}=\frac{N}{2 \cosh x}$, могут быть рассмотрены как сферы Брайанта с гладкими концами. Они также анонсировали, что все уиллморовские сферы являются солитонными сферами (мы отметим, что, согласно результатам Брайанта и Пена, функционал Уиллмора принимает те же самые возможные значения для уиллморовских сфер, что и для сфер Брайанта с гладкими концами [87], [94], [93]). 
5.5. Функционалы уиллморовского типа для поверхностей в трехмерных группах Ли. Формула (44) показывает, что для поверхностей имеет смысл рассматривать функционал

$$
E(\Sigma)=\int_{\Sigma} U V d x \wedge d y
$$

Для торов он измеряет асимптотическую плоскость спектральной кривой и для поверхностей в $\mathbb{R}^{3}$ он равен $E=\frac{1}{4} \mathscr{W}$ [1]. В [11] этот функционал был рассмотрен для поверхностей в других группах Ли и назван энергией поверхности. Хотя произведение $U V$ не всегда имеет вещественные значения, сам функционал для замкнутых поверхностей является вещественным и равен

- для $S U(2)[10]:$

$$
E=\frac{1}{4} \int\left(H^{2}+1\right) d \mu
$$

т.е. кратен функционалу Уиллмора;

- для Nil [11]:

$$
E=\frac{1}{4} \int\left(H^{2}+\frac{\widehat{K}}{4}-\frac{1}{16}\right) d \mu
$$

- для $\widetilde{S L}_{2}[11]$ :

$$
E(M)=\frac{1}{4} \int_{M}\left(H^{2}+\frac{5}{16} \widehat{K}-\frac{1}{4}\right) d \mu ;
$$

- для поверхностей в Sol, поскольку потенциалы имеют неопределенности на множестве нулевой меры, энергия $E$ корректно определена; однако мы не знаем до сих пор ее геометрический смысл.

Напомним, что через $\widehat{K}$ мы обозначаем секционную кривизну объемлющего пространства вдоль касательной плоскости к поверхности.

Эти функционалы не были изучены и многие проблемы остаются открытыми:

1) ограничены ли они снизу (некоторые численные эксперименты подтверждают это)?

2) каковы у них экстремали?

3) каковы аналоги гипотезы Уиллмора для этих функционалов?

\section{Приложение 1. О существовании спектральной кривой оператора Дирака с $L_{2}$-потенциалами}

В этом приложении мы излагаем доказательство теоремы 10, следуя статье [5], где на наш взгляд оно изложено слишком кратко.

Более того, идеи доказательства этой теоремы являются существенными при доказательстве основного результата работы [92]: минимум функционала Уиллмора в заданном конформном классе поверхностей строится следующим образом. Рассмотрим инфимум в данном классе этого функционала и возьмем последовательность поверхностей, а точнее их представлений Вейерштрасса, со значениями функционала Уиллмора, стремящимися к инфимуму. Тогда существует слабо сходящаяся последовательность потенциалов соответствующих операторов Дирака. Оператор Дирака с предельным потенциалом также 
имеет нетривиальное ядро (это следует из сходимости резольвент), и по функции из этого ядра посредством представления Вейерштрасса строится искомая минимизирующая поверхность. Конечно, надо проконтролировать гладкость, что можно. В то же время в [92] отмечено, что, например, отсутствие точек ветвления у предельной поверхности при этом утверждать нельзя.

Аналогом разложения (40) является следующая последовательность:

$$
L_{p} \stackrel{\left(\mathscr{D}_{0}-E_{0}\right)^{-1}}{\longrightarrow} W_{p}^{1 \text { вложение Соболева }} L_{\frac{2 p}{2-p}} \stackrel{\text { умножение }}{\longrightarrow} L_{p}, \quad p<2 .
$$

Все операторы, входящие в нее, только непрерывны, и мы не можем рассуждать как в п. 4.2 .

Пусть $M=\mathbb{C} / \Lambda$ - тор и $z$ - линейная комплексная координата на нем, определенная по модулю решетки $\Lambda$. Через $\rho\left(z_{1}, z_{2}\right)$ мы обозначим расстояние между точками $z_{1}, z_{2} \in M$ в метрике, индуцированной евклидовой метрикой на $\mathbb{C}$ при накрытии $\mathbb{C} \rightarrow \mathbb{C} / \Lambda$.

Следующее предложение выводится из определения резольвенты

$$
\left(\mathscr{D}_{0}-E\right) R_{0}(E)=\delta\left(z-z^{\prime}\right)
$$

прямыми вычислениями.

ПРЕДЛОЖЕНИЕ 16. Резолъвента

$$
R_{0}(E)=\left(\mathscr{D}_{0}-E\right)^{-1}: L_{2} \rightarrow W_{2}^{1} \rightarrow L_{2}
$$

свободного оператора $\mathscr{D}_{0}: L_{2} \rightarrow L_{2}$ является интегралъным оператором

$$
f(z, \bar{z}) \rightarrow\left[R_{0}(E) f\right](z, \bar{z})=\int_{M} K_{0}\left(z, z^{\prime}, E\right) f\left(z^{\prime}, \bar{z}^{\prime}\right) d x^{\prime} d y^{\prime}, \quad z^{\prime}=x^{\prime}+i y^{\prime},
$$

с лдром $K_{0}\left(z, \bar{z}, z^{\prime}, \bar{z}^{\prime}, E\right)=\left(\begin{array}{ll}r_{11} & r_{12} \\ r_{21} & r_{22}\end{array}\right), r_{i k}=r_{i k}\left(z, \bar{z}, z^{\prime}, \bar{z}^{\prime}, E\right)$, где

$r_{12}=\frac{1}{E} \partial r_{22}, \quad r_{21}=-\frac{1}{E} \bar{\partial} r_{11}, \quad \frac{1}{E}\left(\partial \bar{\partial}+E^{2}\right) r_{11}=\frac{1}{E}\left(\partial \bar{\partial}+E^{2}\right) r_{22}=-\delta\left(z-z^{\prime}\right)$.

СледСтвиЕ 8. Интегральное ядро резольвенты $R_{0}(E)$ равно

$$
K_{0}\left(z, z^{\prime}, E\right)=\left(\begin{array}{cc}
-E G & -\partial G \\
\bar{\partial} G & -E G
\end{array}\right)
$$

где $G$ - (модифицированная) функиия Грина оператора Лапласа на торе $M$ :

$$
\left(\partial \bar{\partial}+E^{2}\right) G\left(z, z^{\prime}, E\right)=\delta\left(z-z^{\prime}\right) .
$$

ПримеР. Для тора $M=\mathbb{C} /\{2 \pi \mathbb{Z}+2 \pi i \mathbb{Z}\}$ мы имеем

$$
\begin{gathered}
\delta\left(z-z^{\prime}\right)=\sum_{k, l \in \mathbb{Z}} e^{i\left(k\left(x-x^{\prime}\right)+l\left(y-y^{\prime}\right)\right)}, \quad z=x+i y, \quad z^{\prime}=x^{\prime}+i y^{\prime}, \\
G\left(z, z^{\prime}, E\right)=-4 \sum_{k, l \in \mathbb{Z}} \frac{1}{k^{2}+l^{2}-4 E^{2}} e^{i\left(k\left(x-x^{\prime}\right)+l\left(y-y^{\prime}\right)\right)} .
\end{gathered}
$$

Для других решеток периодов $\Lambda$ аналог ряда (63) для $G$ выглядит почти так же и имеет очень сходные аналитические свойства. Мы не будем его выписывать и будем всегда ссылаться на (63) при рассмотрении его аналитических свойств. 
Следующее предложение очевидно.

ПреДЛОЖЕНИЕ 17. Ряд (63) сходится при $E=i \lambda$, где $\lambda \in \mathbb{R} u \lambda \gg 0$ (m.e. $\lambda$ достаточно велико).

Для вычисления оператора

$$
R_{0}(k, E)=\left(\mathscr{D}_{0}+T_{k}-E\right)^{-1}: L_{2} \rightarrow W_{2}^{1} \stackrel{\text { вложение }}{\longrightarrow} L_{2}
$$

мы используем следующее тождество:

$$
\mathscr{D}_{0}+T_{k}-E=\left(1+T_{k}\left(\mathscr{D}_{0}-E\right)^{-1}\right)\left(\mathscr{D}_{0}-E\right)=\left(1+T_{k} R_{0}(E)\right)\left(\mathscr{D}_{0}-E\right),
$$

которое влечет формулу для резольвенты

$$
R_{0}(k, E)=R_{0}(E)\left(1+T_{k} R_{0}(E)\right)^{-1}=R_{0}(E) \sum_{l=0}^{\infty}\left[-T_{k} R_{0}(E)\right]^{l}
$$

при условии, что ряд в правой части сходится.

ЗАМЕЧАНИЕ. При $p, 1<p<2$, символ

$$
R_{0}(E)=\left(\mathscr{D}_{0}-E\right)^{-1} \quad \text { или } \quad R_{0}(k, E)=\left(\mathscr{D}_{0}+T_{k}-E\right)^{-1}
$$

в разных ситуациях обозначает

а) оператор $A: L_{p} \rightarrow W_{p}^{1}$;

б) композицию $B: L_{p} \rightarrow L_{q}, q=2 p /(2-p)$, оператора $A$ и соболевского вложения $W_{p}^{1} \rightarrow L_{q}$;

в) композицию $C: L_{p} \rightarrow L_{p}$ оператора $A$ и естественного вложения $W_{p}^{1} \rightarrow L_{p}$. Действие этих операторов совпадает на пространстве гладких функций, которые можно рассматривать, как вложенные в $W_{p}^{1}$ или $L_{q}$ (все эти пространства - это замыкания пространства гладких функций по отношению к различным нормам). Следовательно, достаточно продемонстрировать и доказать все необходимые оценки только для гладких функций, и это можно сделать при помощи явных формул для резольвент.

Разложим резольвенты в следующие суммы интегральных операторов.

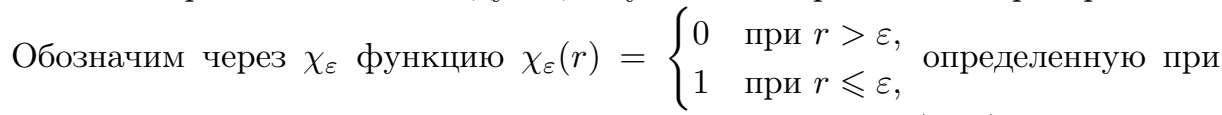
$r \geqslant 0, r \in \mathbb{R}$. При заданном $\delta>0$ разложим резольвенту $R_{0}(k, E)$ в сумму двух интегральных операторов

$$
R_{0}(k, E)=R_{0}^{\leqslant \varepsilon}(k, E)+R_{0}^{>\varepsilon}(k, E): L_{p} \rightarrow L_{q},
$$

где “ближняя" часть $R_{0}^{\leqslant \varepsilon}(k, E)$ определяется ее ядром

$$
K_{0}^{\leqslant \varepsilon}\left(z, \bar{z}, z^{\prime}, \bar{z}^{\prime}, E\right)=K_{0}\left(z, \bar{z}, z^{\prime}, \bar{z}^{\prime}, E\right) \chi_{\varepsilon}\left(\rho\left(z, z^{\prime}\right)\right)
$$

и “дальняя" $R_{0}^{>\varepsilon}(k, E)$ имеет следующее ядро:

$$
K_{0}^{>\varepsilon}\left(z, \bar{z}, z^{\prime}, \bar{z}^{\prime}, E\right)=K_{0}\left(z, \bar{z}, z^{\prime}, \bar{z}^{\prime}, E\right)\left(1-\chi_{\varepsilon}\left(\rho\left(z, z^{\prime}\right)\right)\right) .
$$


ПреДЛОЖЕНИЕ 18. Для заданнъх $p, 1<p<2, \hat{k}=\left(\hat{k}_{1}, \hat{k}_{2}\right) \in \mathbb{C}^{2} u \delta$, $0<\delta<1$, существует такая вещественная постоянная $\lambda_{0} \gg 0$, что

$$
\left\|T_{k} R_{0}(i \lambda)\right\|_{L_{p} \rightarrow L_{p}}<\delta
$$

для всех $\lambda>\lambda_{0} u k$, достаточно близких $\kappa \hat{k}$.

Следовательно, для таких $\lambda$ и $k$

1) ряд из (64) сходится и определяет ограниченный оператор из $L_{p}$ в $W_{p}^{1}$, $L_{q}$ или $L_{p}$ (в зависимости от того, какой смысл имеет символ $R_{0}(E)$, умноженный слева на ряд);

2) норма оператора

$$
R_{0}(k, i \lambda): L_{p} \rightarrow W_{p}^{1}
$$

ограничена некоторой постоянной $r_{p}$;

3) для заданного $\varepsilon>0$ мы имеем

$$
\lim _{\lambda \rightarrow \infty}\left\|R_{0}^{>\varepsilon}(k, i \lambda)\right\|_{L_{p} \rightarrow L_{q}}=0, \quad q=\frac{2 p}{2-p} .
$$

Это предложение вытекает из явной формулы (63) для ядра резольвенты.

Обозначим через $r_{\text {inj }}$ радиус инъективности метрики на $M$ и введем нормы $\|\cdot\|_{2 ; \varepsilon}$, определенные при $0<\varepsilon<r_{\text {inj }}$, следующим образом. Для $U \in L_{2}(M)$ мы обозначим через $\left.U\right|_{B(z, \varepsilon)}$ ограничение функции $U$ на шар $B(z, \varepsilon)=\{w \in M$ : $\rho(z, w)<\varepsilon\}$ и определим $\|U\|_{2 ; \varepsilon}$ как

$$
\|U\|_{2 ; \varepsilon}=\max _{z \in M}\left\|\left.U\right|_{B(z, \varepsilon)}\right\|_{2}
$$

ПреДЛОЖЕНИЕ 19. 1) Для всех $U \in L_{2}(M)$ выполняются неравенства

$$
\sqrt{\frac{\pi \varepsilon^{2}}{\operatorname{vol}(M)}} \leqslant\|U\|_{2 ; \varepsilon} \leqslant\|U\|_{2} .
$$

2) Для всех $C>0$ и м множества $\left\{\|U\|_{2 ; \varepsilon} \leqslant C\right\}$ замкнуты и, следователъно, компактны и в слабой топологии, и в топологии слабой сходимости на $L_{2}(M)$.

ДоказАтельство. Очевидно, что $\|U\|_{2 ; \varepsilon} \leqslant\|U\|_{2}$. Более того, мы имеем

$$
\begin{aligned}
\|U\|_{2 ; \varepsilon}^{2} \operatorname{vol}(M) & \geqslant \int_{M} \int_{B(z, \varepsilon)}\left|U\left(z+z^{\prime}, \bar{z}+\bar{z}^{\prime}\right)\right|^{2} d z^{\prime} d z \\
& =\int_{M} \int_{B(0, \varepsilon)}\left|U\left(z+z^{\prime}, \bar{z}+\bar{z}^{\prime}\right)\right|^{2} d z^{\prime} d z \\
& =\int_{B(0, \varepsilon)}\left[\int_{M}|U(z, \bar{z})|^{2} d z\right] d z^{\prime}=\pi \varepsilon^{2}\|U\|_{2}^{2},
\end{aligned}
$$

где $d z=d x \wedge d y, d z^{\prime}=d x^{\prime} \wedge d y^{\prime}$. Второе утверждение известно из курса функционального анализа.

Рассмотрим резольвенту

$$
R(k, E)=\left(\mathscr{D}+T_{k}-E\right)^{-1}: L_{p} \rightarrow L_{p}
$$


Мы опять используем тождество

$$
\mathscr{D}+T_{k}-E=\left[1+\left(\begin{array}{cc}
U & 0 \\
0 & V
\end{array}\right)\left(\mathscr{D}_{0}+T_{k}-E\right)^{-1}\right]\left(\mathscr{D}_{0}+T_{k}-E\right),
$$

которое влечет равенство

$$
R(k, E)=R_{0}(k, E) \sum_{l=0}^{\infty}\left[-\left(\begin{array}{cc}
U & 0 \\
0 & V
\end{array}\right) R_{0}(k, E)\right]^{l} .
$$

ПреДЛОЖЕНИЕ 20. Пустъ $1<p<2, \hat{k}=\left(\hat{k}_{1}, \hat{k}_{2}\right) \in \mathbb{C}^{2}, \varepsilon$ достаточно мало u $0<\delta<1$. Существует такое $\gamma>0$, ито

$$
\left\|\left(\begin{array}{cc}
U & 0 \\
0 & V
\end{array}\right) R_{0}(k, i \lambda)\right\|_{L_{p} \rightarrow L_{p}}<\delta
$$

для всех $\lambda>\lambda_{0}, k$, достаточно близких $\kappa \hat{k}, u U, V \operatorname{ma\kappa ux,~что~}\|U\|_{2 ; \varepsilon}<\gamma$, $\|V\|_{2 ; \varepsilon}<\gamma$.

ДокАзАТЕЛьство. Имеет место очевидное неравенство

$$
\left\|R_{0}^{\leqslant \varepsilon}(k, E)\right\| \leqslant\left\|R_{0}(k, E)\right\| \quad \text { при всех } \varepsilon .
$$

Пусть $S_{p}-$ постоянная Соболева для вложения $W_{p}^{1} \rightarrow L_{q}$ (см. предложение 5 ). При $\lambda>\lambda_{0}$ мы имеем

$$
\left\|R_{0}(k, i \lambda)\right\|_{L_{p} \rightarrow W_{p}^{1}} \leqslant r_{p}
$$

(см. предложение 18). Рассмотрим теперь композицию отображений

$$
L_{p} \stackrel{R_{0}^{\leqslant \varepsilon}(k, E)}{\longrightarrow} W_{p}^{1} \stackrel{\text { вложение }}{\longrightarrow} L_{q} \stackrel{\times\left(\begin{array}{cc}
U & 0 \\
0 & V
\end{array}\right)}{\longrightarrow} L_{p},
$$

где норма первого из них ограничена сверху постоянной $r_{p}$, а норма второго постоянной $S_{p}$. Вычислим норму третьего отображения.

Так как интегральное ядро оператора $R_{0}^{\leqslant \varepsilon}(k, E)$ локализовано в замкнутой области $\left\{\rho\left(z, z^{\prime}\right) \leqslant \varepsilon\right\}$, для любого шара $B(x, \alpha)$ мы имеем

$$
\left.\left[\left(\begin{array}{cc}
U & 0 \\
0 & V
\end{array}\right) R_{0}^{\leqslant \varepsilon}(k, E) f\right]\right|_{B(x, \alpha)}=\left[\left(\begin{array}{cc}
U & 0 \\
0 & V
\end{array}\right) R_{0}^{\leqslant \varepsilon}(k, E)\right]\left(\left.f\right|_{B(x, \alpha+\varepsilon)}\right) .
$$

Применяя неравенство Гёльдера к правой части этой формулы, мы получаем

$$
\begin{aligned}
& \left\|\left.\left[\left(\begin{array}{cc}
U & 0 \\
0 & V
\end{array}\right) R_{0}^{\leqslant \varepsilon}(k, E) f\right]\right|_{B(x, \alpha)}\right\|_{p} \\
& \quad \leqslant m\left\|R_{0}^{\leqslant \varepsilon}(k, E)\right\|_{L_{p} \rightarrow L_{q}}\left\|\left(\left.f\right|_{B(x, \alpha+\varepsilon)}\right)\right\|_{p} \leqslant m r_{p} S_{p}\left\|\left(\left.f\right|_{B(x, \alpha+\varepsilon)}\right)\right\|_{p},
\end{aligned}
$$

где $m=\max \left(\|U\|_{2 ; \varepsilon},\|V\|_{2 ; \varepsilon}\right)$. Напомним теперь тождество

$$
\int_{M}\left\|g_{B(x, \alpha)}\right\|_{p}^{p} d x=\operatorname{vol} B(x, \alpha)\|g\|_{p}^{p}=\pi \alpha^{2}\|g\|_{p}^{p}
$$


и, применив его к предыдущему неравенству, получим

$$
\left\|\left(\begin{array}{cc}
U & 0 \\
0 & V
\end{array}\right) R_{0}^{\leqslant \varepsilon}(k, E)\right\|_{p} \leqslant m r_{p} S_{p}\left(1+\frac{\varepsilon}{\alpha}\right)^{2 / p} .
$$

Так как мы используем постоянную Соболева $S_{p}$ для тора, мы должны предположить, что $(\alpha+\varepsilon)<r_{\mathrm{inj}}$. Если

$$
m=\max \left(\|U\|_{2 ; \varepsilon},\|V\|_{2 ; \varepsilon}\right)<\frac{\delta}{r_{p} S_{p} \sqrt[p]{4}}
$$

и $\alpha=\varepsilon<r_{\text {inj }} / 2$, мы имеем

$$
\left\|\left(\begin{array}{cc}
U & 0 \\
0 & V
\end{array}\right) R_{0}^{\leqslant \varepsilon}(k, E)\right\|_{p}<\delta .
$$

Предложение доказано.

Предложение 21 [5]. Пусть р $и \hat{k} \in \mathbb{C}^{2}$ те же самые, что и в предложении 20, $\gamma<\left(r_{p} S_{p} \sqrt[p]{4}\right)^{-1}$ и $\lambda \gg 0$, m.е. $\lambda$ достаточно велико. Пусть задано достаточно малое $\varepsilon>0$. Для таких $U, V$, что $\|U\|_{2 ; \varepsilon} \leqslant C \leqslant \gamma,\|V\|_{2 ; \varepsilon} \leqslant C \leqslant \gamma$, ряд

$$
R(k, i \lambda)=R_{0}(k, i \lambda) \sum_{l=0}^{\infty}\left[-\left(\begin{array}{cc}
U & 0 \\
0 & V
\end{array}\right) R_{0}(k, i \lambda)\right]^{l}
$$

равномерно сходится около $\hat{k}$ и определяет резольвенту оператора

$$
\mathscr{D}+T_{k}: L_{p} \rightarrow L_{p}
$$

Действие этой резольвенты на гладких функциях продолжается до резольвенты оператора $\mathscr{D}+T_{k}$ на пространстве $L_{2}$ :

$$
\left(\mathscr{D}+T_{k}-E\right)^{-1}: L_{2} \rightarrow W_{2}^{1} \stackrel{\text { вложение }}{\longrightarrow} L_{2} \text {. }
$$

Этот пучок компактных операторов голоморфен по $k$ около $\hat{k}$. Если $\left(U_{n}, V_{n}\right)$ $\stackrel{\text { слабо }}{\longrightarrow}\left(U_{\infty}, V_{\infty}\right)$ в $\left\{\|U\|_{2 ; \varepsilon} \leqslant C,\|V\|_{2 ; \varepsilon}<C\right\}$, то соответствующие резолъвенты сходятся по норме $к$ резольвенте оператора с потенииалами $\left(U_{\infty}, V_{\infty}\right)$.

ДокАЗАТЕЛЬСтво. Согласно предложению 20 и (65), при $\lambda>\lambda_{0}$ около $\hat{k}$ выполняется неравенство

$$
\left\|\left(\begin{array}{cc}
U & 0 \\
0 & V
\end{array}\right) R_{0}^{\leqslant \varepsilon}(k, i \lambda)\right\|_{p}<\sigma=\gamma r_{p} S_{p} \sqrt[p]{4}<1 .
$$

Согласно предложению 18, для достаточно больших вещественных значений $\lambda$, так как норма вложения $L_{q} \rightarrow L_{p}$ ограничена, мы имеем

$$
\left\|\left(\begin{array}{cc}
U & 0 \\
0 & V
\end{array}\right) R_{0}^{>\varepsilon}(k, i \lambda)\right\|_{p}<1-\sigma .
$$

Это влечет, что для $\lambda \gg 0$ имеет место неравенство

$$
\left\|\left(\begin{array}{cc}
U & 0 \\
0 & V
\end{array}\right) R_{0}(k, i \lambda)\right\|_{p} \leqslant\left\|\left(\begin{array}{cc}
U & 0 \\
0 & V
\end{array}\right)\left(R_{0}^{\leqslant \varepsilon}(k, i \lambda)+R_{0}^{>\varepsilon}(k, i \lambda)\right)\right\|_{p}<1
$$


и ряд (66) равномерно сходится около $\hat{k}$ и задает резольвенту оператора $\mathscr{D}+T_{k}: L_{p} \rightarrow L_{p}$.

Действие $R(k, i \lambda)$ на гладких функциях дается формулой $(66)$, и мы продолжим его до компактного оператора на $L_{2}$ следующим образом. Положим

$$
B=\sum_{l=0}^{\infty}\left[-\left(\begin{array}{cc}
U & 0 \\
0 & V
\end{array}\right) R_{0}(k, i \lambda)\right]^{l}
$$

и рассмотрим следующую композицию операторов:

$$
L_{2} \stackrel{\text { вложение }}{\longrightarrow} L_{p} \stackrel{B}{\rightarrow} L_{p} \stackrel{\left(\mathscr{D}+T_{k}-E\right)^{-1}}{\longrightarrow} W_{p}^{1} \stackrel{\text { вложение }}{\longrightarrow} L_{2},
$$

где все операторы ограничены и вложение $W_{p}^{1} \rightarrow L_{2}$ компактно согласно теореме Кондрашова (см. предложение 5). Это показывает, что действие $R(k, i \lambda)$ на гладких функциях продолжается до компактного оператора на $L_{2}$. Так как ряд (66) голоморфен по $k$, резольвента $R(k, i \lambda)$ тоже голоморфна по $k$.

Нам осталось доказать, что резольвента непрерывна по $U$ и $V$. Каждое вхождение матрицы $\left(\begin{array}{cc}U & 0 \\ 0 & V\end{array}\right)$ в член ряда (66) окружено с обеих сторон резольвентами $R_{0}(k, i \lambda)$, которые являются ограниченными интегральными операторами. Пусть $l=1$ и $K\left(z, z^{\prime}, k, i \lambda\right)$ - ядро такого оператора. Тогда композиция

$$
R_{0}(k, i \lambda)\left(\begin{array}{cc}
U & 0 \\
0 & V
\end{array}\right) R_{0}(k, i \lambda)
$$

действует на гладких функциях как интегральный оператор с ядром

$$
F\left(z, z^{\prime \prime}\right)=K\left(z, z^{\prime}, k, i \lambda\right)\left(\begin{array}{cc}
U\left(z^{\prime}\right) & 0 \\
0 & V\left(z^{\prime}\right)
\end{array}\right) K\left(z^{\prime}, z^{\prime \prime}, k, i \lambda\right) .
$$

Очевидно, что такой интегральный оператор непрерывен относительно слабой сходимости потенциалов $U, V \in L_{2}(M)$. Для других значений $l$ доказательство аналогично. Согласно предложению 19, каждый член ряда (66) непрерывен относительно слабой сходимости потенциалов в $\left\{\|U\|_{2 ; \varepsilon} \leqslant C,\|V\|_{2 ; \varepsilon} \leqslant C\right\}$. Так как ряд (66) сходится равномерно, то это свойство непрерывности выполняется и для суммы ряда. Предложение доказано.

Это предложение устанавливает существование резольвенты только для больших значений $\lambda$, где $E=i \lambda$. Резольвента продолжается до мероморфной функции на $E$-плоскости с помощью формулы Гильберта (см. предложение 6 ).

ДоКАЗАТЕЛЬСТВо тЕОРЕмЫ 10. Согласно предложению 21, существуют такие $k_{0} \in \mathbb{C}^{2}$ и $E \in \mathbb{C}$, что оператор

$$
\left(\mathscr{D}+T_{k_{0}}-E_{0}\right)^{-1}: L_{2} \rightarrow W_{2}^{1} \stackrel{\text { вложение }}{\longrightarrow} L_{2}
$$

корректно определен. Подставим выражение $\varphi=\left(\mathscr{D}+T_{k_{0}}-E\right)^{-1} f$ в уравнение

$$
\left(\mathscr{D}+T_{k}-E\right) \varphi=0
$$

и перепишем это уравнение в виде

$$
\left(\mathscr{D}+T_{k_{0}}-T_{k_{0}}+T_{k}-E_{0}+E_{0}-E\right)\left(\mathscr{D}+T_{k_{0}}-E\right)^{-1} f=\left[1+A_{U, V}(k, E)\right] f=0,
$$


где

$$
A_{U, V}(k, E)=\left(T_{k}-T_{k_{0}}+E_{0}-E\right)\left(\mathscr{D}+T_{k_{0}}-E\right)^{-1} .
$$

Так как первый сомножитель в этой формуле - ограниченный оператор для всех $k, E$ и второй сомножитель - компактный оператор, то $A_{U, V}(k, E)$ - пучок компактных операторов, полиномиальных по $k$ и $E$. Применяя теорему Келдыша так же, как в п. 4.2, мы завершаем доказательство теоремы.

Теперь спектральная кривая, как обычно, определяется формулой

$$
\Gamma=Q_{0}(U, V) / \Lambda^{*}
$$

ЗАмечание. Резольвенты операторов на некомпактных пространствах не зависят непрерывно от потенциалов при их слабой сходимости. Действительно, рассмотрим оператор Шрёдингера

$$
L=-\frac{d^{2}}{d x^{2}}+U(x)
$$

где $U(x)$ - солитонный потенциал (следовательно, оператор имеет связанные состояния). Изоспектральная последовательность потенциалов $U_{N}(x)=$ $U(x+N)$ слабо сходится к нулевому потенциалу $U_{\infty}=0$, для которого оператор Шрёдингера не имеет связанных состояний. Это же верно для одномерного оператора Дирака.

\section{Приложение 2. Конформное отображение Гаусса и конформная площадь}

В этом приложении мы изложим известные результаты о конформном отображении Гаусса, следуя в основном [87], [133], [109].

Обозначим через $S_{q, r}$ сферу радиуса $r$ в $\mathbb{R}^{3}$ с центром в точке $q$ и через $\Pi_{p, N}$ плоскость в $\mathbb{R}^{3}$, проходящую через точку $p$ и с нормальным вектором $N$. Все такие сферы и плоскости параметризуются квадрикой $Q^{4} \subset \mathbb{R}^{4,1}$. Действительно, пусть

$$
\langle x, y\rangle=x_{1} y_{1}+\cdots+x_{4} y_{4}-x_{5} y_{5}
$$

- скалярное произведение в $\mathbb{R}^{4,1}$. Положим

$$
\begin{gathered}
Q^{4}=\{\langle x, x\rangle=1\} \subset \mathbb{R}^{4,1}, \\
S_{q, r} \rightarrow \frac{1}{r}\left(q, \frac{1}{2}\left(|q|^{2}-r^{2}-1\right), \frac{1}{2}\left(|q|^{2}-r^{2}+1\right)\right), \\
\prod_{q, N} \rightarrow(N,\langle q, N\rangle,\langle q, N\rangle) .
\end{gathered}
$$

Для поверхности $f: \Sigma \rightarrow \mathbb{R}^{3}$ ее конформное гауссово отображение

$$
G^{c}: \Sigma \rightarrow Q^{4}
$$

сопоставляет точке $p \in \Sigma$ сферу радиуса $1 / H$, касающуюся поверхности в точке $p$, при $H \neq 0$ :

$$
G^{c}(p)=S_{p+N / H, 1 / H},
$$

и касательную плоскость в этой точке при $H \neq 0$. В координатах на $Q^{4}$ оно имеет вид

$$
G^{c}(p)=H \cdot X+T
$$


где

$$
X=\left(f, \frac{(f, f)-1}{2}, \frac{(f, f)+1}{2}\right), \quad T=(N,(N, f),(N, f)) .
$$

Это отображение - специальный случай так называемых сферических конгруэнций, являющихся одной из основных тем конформной геометрии (современное состояние этой теории представлено в [134]).

Мы имеем $\langle X, X\rangle=0,\langle T, T\rangle=1$ и $\langle X, T\rangle=0$, что влечет $\langle d X, X\rangle=$ $\langle d T, T\rangle=0,\langle d T, X\rangle=\langle-d X, T\rangle$. Легко проверить, что

$$
\begin{gathered}
\langle d X, T\rangle=(d f, N)=0, \quad\langle d X, D X\rangle=(d f, d f)=\mathbf{I}, \\
\langle d X, d T\rangle=(d f, d N)=-\mathbf{I I}, \quad\langle d T, d T\rangle=(d N, d N)=\mathbf{I I I},
\end{gathered}
$$

где третья квадратичная форма поверхности (обозначим ее III) измеряет длины образов кривых при отображении Гаусса и удовлетворяет соотношению

$$
K \cdot \mathbf{I}-2 H \cdot \mathbf{I I}+\mathbf{I I I}=0
$$

связывающему ее с первой и второй квадратичными формами поверхности $\mathbf{I}$ и II. Отсюда следует, что

$$
\left\langle Y_{z}, Y_{z}\right\rangle=\left\langle Y_{\bar{z}}, Y_{\bar{z}}\right\rangle=0, \quad\left\langle Y_{z}, Y_{\bar{z}}\right\rangle=e^{\beta}=\frac{\left(H^{2}-K\right) e^{2 \alpha}}{2}=\left(H^{2}-K\right)\left(f_{z}, f_{\bar{z}}\right)
$$

где для краткости мы обозначили $G^{c}$ через $Y, z$ - конформный параметр на поверхности и $\mathbf{I}=e^{2 \alpha} d z d \bar{z}-$ индуцированная метрика на поверхности. Мы заключаем, что

конформное гауссово отображение регулярно и конформно вне омбилических точек.

Очевидно, что векторы $X$ и $Y$ линейно независимы и вне омбилических точек множество векторов $Y, Y_{z}, Y_{\bar{z}}, X$ однозначно пополняется вектором $Z \in \mathbb{R}^{5}$ до такого базиса

$$
\sigma=\left(Y, Y_{z}, Y_{\bar{z}}, X, Z\right)^{T}
$$

в $\mathbb{C}^{5}$ - комплексификации пространства $\mathbb{R}^{5},-$ что скалярное произведение в $\mathbb{R}^{4,1}$ принимает вид

$$
\left(\begin{array}{ccccc}
1 & 0 & 0 & 0 & 0 \\
0 & 0 & e^{\beta} & 0 & 0 \\
0 & e^{\beta} & 0 & 0 & 0 \\
0 & 0 & 0 & 0 & 1 \\
0 & 0 & 0 & 1 & 0
\end{array}\right)
$$


Аналоги уравнений Гаусса-Вейнгартена имеют вид

$$
\begin{gathered}
\sigma_{z}=\mathbf{U} \sigma, \\
\mathbf{U}=\left(\begin{array}{ccccc}
0 & 1 & 0 & 0 & 0 \\
0 & \beta_{z} & 0 & C_{2} & C_{1} \\
-e^{\beta} & 0 & 0 & C_{4} & C_{3} \\
0 & -e^{-\beta} C_{3} & -e^{-\beta} C_{1} & C_{5} & 0 \\
0 & -e^{-\beta} C_{4} & -e^{-\beta} C_{2} & 0 & -C_{5}
\end{array}\right), \\
\mathbf{V}=\left(\begin{array}{ccccc}
0 & 0 & 1 & 0 & 0 \\
-e^{\beta} & 0 & 0 & C_{4} & C_{3} \\
0 & 0 & \beta \bar{z} & \bar{C}_{2} & \bar{C}_{1} \\
0 & -e^{-\beta} \bar{C}_{1} & -e^{-\beta} \bar{C}_{3} & \bar{C}_{5} & 0 \\
0 & -e^{-\beta} \bar{C}_{2} & -e^{-\beta} \bar{C}_{4} & 0 & -\bar{C}_{5}
\end{array}\right),
\end{gathered}
$$

где

$$
\begin{gathered}
C_{1}=\left\langle Y_{z z}, X\right\rangle_{4,1}, \quad C_{2}=\left\langle Y_{z z}, Z\right\rangle_{4,1}, \quad C_{3}=\left\langle Y_{z \bar{z}}, X\right\rangle_{4,1}, \\
C_{4}=\left\langle Y_{z \bar{z}}, Z\right\rangle_{4,1}, \quad C_{5}=\left\langle X_{z}, Z\right\rangle_{4,1} .
\end{gathered}
$$

Прямыми вычислениями проверяется тождество

$$
\Delta Y+2\left(H^{2}-K\right) Y=\left(\Delta H+2 H\left(H^{2}-K\right)\right) X,
$$

из которого, в частности, вытекает, что

$$
C_{3}=0, \quad C_{4}=\frac{e^{2 \alpha}}{4}\left(\Delta H+2 H\left(H^{2}-K\right)\right) .
$$

Здесь $\Delta=4 e^{-2 \alpha} \partial \bar{\partial}$ обозначает оператор Лапласа-Бельтрами на поверхности. Учитывая это и то, что величина $C_{4}$ вещественнозначна, мы выводим уравнения Кодацци для конформного гауссова отображения:

$$
\begin{gathered}
\beta_{z \bar{z}}+e^{\beta}-\left(\bar{C}_{1} C_{2}+C_{1} \bar{C}_{2}\right) e^{-\beta}=0, \quad C_{1 \bar{z}}=C_{1} \bar{C}_{5}, \\
C_{2 \bar{z}}+C_{2} \bar{C}_{5}=C_{4 z}-\beta_{z} C_{4}+C_{4} C_{5}, \quad C_{5 \bar{z}}-\bar{C}_{5 z}=e^{-\beta}\left(C_{1} \bar{C}_{2}-\bar{C}_{1} C_{2}\right) .
\end{gathered}
$$

Прямыми вычислениями мы получаем, что

$$
C_{1}=A=\left\langle N, f_{z z}\right\rangle, \quad e^{\beta}=2|A|^{2} e^{-2 \alpha} .
$$

Конформная площадъ $V^{c}$ поверхности $\Sigma$ - это площадь ее образа в $Q^{4}$ :

$$
V^{c}(\Sigma)=\int_{\Sigma}\left(H^{2}-K\right) d \mu
$$

где $d \mu$ - форма площади на $\Sigma$. Уравнения Эйлера-Лагранжа для $V^{c}$ принимают вид

$$
\Delta H+2 H\left(H^{2}-K\right)=0 .
$$

Поверхность в $\mathbb{R}^{3}$ называется конформно минимальной (или поверхностью Уиллмора), если она удовлетворяет этому уравнению. Мы заключаем, что конформно минимальные поверхности - это в точности те поверхности, чьи образы при конформном гауссовом отображении $G^{c}$ являются минимальными поверхностями в $Q^{4}$. 
Для неомбилической точки $p \in \Sigma$ касательное пространство к $Q^{4}$ в точке $Y(p)$ порождено векторами $Y_{z}, Y_{\bar{z}}, X$ и $Z$. Мы видим, что отображение $Y$ конформно гармоническое, т.е. $\Delta Y$ всюду ортогонально касательной плоскости к $Q^{4}$, если и только если поверхность является конформно минимальной.

Из уравнений Гаусса-Вейнгартена для $G^{c}$ и уравнений Эйлера-Лагранжа для $V^{c}$ следует, что если $\Sigma$ конформно минимальна, т.е. $C_{4}=0$, то дифференциал четвертой степени

$$
\omega=\left\langle Y_{z z}, Y_{z z}\right\rangle(d z)^{4}=C_{1} C_{2}(d z)^{4}
$$

является голоморфным.

Напомним, что голоморфный дифференциал четвертой степени на двумерной сфере равен нулю: $\omega=0$, а на торе имеет постоянные коэффициенты: $\omega=$ const $\cdot(d z)^{4}$.

Минимальная поверхность в $Q^{4}$ называется суперминималъной, если $\omega=0$. Положим

$$
\varphi=\log \frac{\bar{C}_{1}}{C_{1}} .
$$

Заметим, что $C_{1} \equiv 0$ только для вполне омбилических поверхностей и по теореме Хопфа это - области в круглой сфере из $\mathbb{R}^{3}$ либо на плоскости.

Если $\omega \equiv 0$ и $C_{1} \neq 0$, то $C_{2} \equiv 0$ и уравнения Гаусса-Кодацци для конформного гауссова отображения сводятся к

$$
\beta_{z \bar{z}}+e^{\beta}=0, \quad \varphi_{z \bar{z}}=0 .
$$

Первое из этих уравнений - это уравнение Лиувилля, а второе - уравнение Лапласа. Эти уравнения описывают суперминимальные не вполне омбилические поверхности.

Рассмотрим случай, когда конформно минимальная поверхность не суперминимальна. Локально заменой конформного параметра можно свести все к случаю

$$
\frac{1}{2}\left\langle Y_{z z}, Y_{z z}\right\rangle_{4,1}=C_{1} C_{2}=\frac{1}{2}
$$

Тогда уравнения Гаусса-Кодацци принимают вид четырехчастичной решетки Тоды

$$
\beta_{z \bar{z}}+e^{\beta}-e^{-\beta} \cosh \varphi=0, \quad \varphi_{z \bar{z}}+e^{-\beta} \sinh \varphi=0
$$

\section{Приложение 3. Обратная задача рассеяния для оператора Дирака на прямой и формулы следов}

Здесь, ориентируясь в основном на геометров, мы изложим некоторые факты, необходимые для доказательства теоремы 13 и введения солитонных сфер в п. 5.4. Это - сокращенная версия приложения к электронной версии работы [6] (cм. http://arxiv.org/math.DG/9801022), где теорема 13 была изначально доказана.

Обратная задача рассеяния для оператора Дирака на прямой была решена в [135] аналогично той же задаче для оператора Шрёдингера $-\partial_{x}^{2}+u(x)[136]$ (см. также [137]). 
Рассмотрим следующую спектральную задачу (известную как задача Захарова-Шабата):

$$
L \psi=\left(\begin{array}{cc}
0 & i k \\
i k & 0
\end{array}\right) \psi
$$

где

$$
L=\left(\begin{array}{cc}
0 & \partial_{x} \\
-\partial_{x} & 0
\end{array}\right)+\left(\begin{array}{ll}
p & 0 \\
0 & q
\end{array}\right)
$$

Мы предполагаем, что потенциалы $p$ и $q$ быстро убывают при $x \rightarrow \pm \infty$. Из доказательств ясно, что достаточно предположить, что потенциалы $p(x)$ и $q(x)$ убывают экспоненциально.

При $p=q=0$ для каждого $k \in \mathbb{R} \backslash\{0\}$ мы имеем двумерное пространство решений (свободных волн), порожденное столбцами матрицы

$$
\Phi_{0}(x, k)=\left(\begin{array}{cc}
0 & e^{-i k x} \\
e^{i k x} & 0
\end{array}\right) .
$$

Для нетривиальных $p$ и $q$ для каждого $k \in \mathbb{R} \backslash\{0\}$ мы опять имеем двумерное пространство решений, которые асимптотически ведут себя как свободные волны при $x \rightarrow \pm \infty$. Эти пространства порождены так называемыми функциями Йоста $\varphi_{l}^{ \pm}, l=1,2$. Для определения этих функций мы рассмотрим матрицы $\Phi^{+}(x, k)$ и $\Phi^{-}(x, k)$, удовлетворяющие интегральным уравнениям

$$
\begin{aligned}
& \Phi^{+}(x, k)=\Phi_{0}(x, k)+\int_{x}^{+\infty} \Phi_{0}\left(x-x^{\prime}, k\right)\left(\begin{array}{ll}
p & 0 \\
0 & q
\end{array}\right) \Phi^{+}\left(x^{\prime}, k\right) d x^{\prime} \\
& \Phi^{-}(x, k)=\Phi_{0}(x, k)+\int_{-\infty}^{x} \Phi_{0}\left(x-x^{\prime}, k\right)\left(\begin{array}{ll}
p & 0 \\
0 & q
\end{array}\right) \Phi^{-}\left(x^{\prime}, k\right) d x^{\prime} .
\end{aligned}
$$

Эти уравнения имеют вид $\Phi^{ \pm}=\Phi_{0}+A^{ \pm} \Phi^{ \pm}, A^{ \pm}$- вольтерровские операторы, и, следовательно, каждое из этих уравнений имеет единственное решение, данное рядом Неймана $\Phi^{ \pm}(x, k)=\sum_{l=0}^{\infty}\left(A^{ \pm}\right)^{l} \Phi_{0}(x, k)$. Столбцы матриц $\Phi^{ \pm}$есть функции Йоста $\varphi_{l}^{ \pm}, l=1,2$. Мы видим, что, по построению, функции Йоста ведут себя как свободные волны:

$$
\varphi_{1}^{ \pm} \approx\left(\begin{array}{c}
0 \\
e^{i k x}
\end{array}\right), \quad \varphi_{2}^{ \pm} \approx\left(\begin{array}{c}
e^{-i k x} \\
0
\end{array}\right) \quad \text { при } \quad x \rightarrow \pm \infty
$$

Прямыми вычислениями показывается, что

- для пары решений $\theta=\left(\begin{array}{l}\theta_{1} \\ \theta_{2}\end{array}\right)$ и $\tau=\left(\begin{array}{l}\tau_{1} \\ \tau_{2}\end{array}\right)$ уравнения (68) вронскиан $W=\theta_{1} \tau_{2}-\theta_{2} \tau_{1}$ является постоянным; в частности, мы имеем

$$
\operatorname{det} \Phi^{ \pm}(x, k)=-1
$$

В дальнейшем мы предполагаем, что потенциалы $p$ и $q$ комплексно сопряжены:

$$
p=\bar{q}
$$

Прямыми вычислениями также проверяется, что преобразование

$$
\psi=\left(\begin{array}{c}
\psi_{1} \\
\psi_{2}
\end{array}\right) \rightarrow \psi^{*}=\left(\begin{array}{c}
-\bar{\psi}_{2} \\
\bar{\psi}_{1}
\end{array}\right)
$$


переводит решения уравнения (68) в решения того же самого уравнения. В частности, из асимптотик функций Йоста вытекает, что эти функции преобразуются следующим образом:

$$
\varphi_{1}^{ \pm} \stackrel{*}{\longrightarrow}-\varphi_{2}^{ \pm}, \quad \varphi_{2}^{ \pm} \stackrel{*}{\longrightarrow} \varphi_{1}^{ \pm} .
$$

Поскольку функции Йоста $\varphi_{l}^{+}, l=1,2$, и $\varphi_{l}^{-}, l=1,2$, задают базисы для одного и того же пространства, они связаны преобразованием

$$
\left(\begin{array}{l}
\varphi_{1}^{-} \\
\varphi_{2}^{-}
\end{array}\right)=S(k)\left(\begin{array}{c}
\varphi_{1}^{+} \\
\varphi_{2}^{+}
\end{array}\right)
$$

Из (70) следует, что $\operatorname{det} S(k)=1$, и теперь мы выводим из (72), что - матрица рассеяния $S(k)$ унитарна: $S(k) \in S U(2)$, т.е.

$$
S(k)=\left(\begin{array}{cc}
\overline{a(k)} & -\overline{b(k)} \\
b(k) & a(k)
\end{array}\right), \quad|a(k)|^{2}+|b(k)|^{2}=1 .
$$

Величины

$$
T(k)=\frac{1}{a(k)}, \quad R(k)=\frac{b(k)}{a(k)}
$$

называются коэффициентом прохождения и коэфбициентом отражения соответственно. Оператор называется $L$ безотражательным, если его коэффициент отражения равен нулю: $R(k) \equiv 0$.

Вектор-функции $\varphi_{1}^{-} e^{-i k x}$ и $\varphi_{2}^{+} e^{i k x}$ аналитически продолжаются на нижнюю полуплоскость $\operatorname{Im} k<0$, а вектор-функции $\varphi_{2}^{-} e^{i k x}$ и $\varphi_{1}^{+} e^{-i k x}$ аналитически продолжаются на верхнюю полуплоскость $\operatorname{Im} k>0$.

Без ограничения общности это достаточно доказать для $\varphi_{1}^{-} e^{-i k x}$. Эта функция удовлетворяет уравнению типа Вольтерра:

$$
f(x, k)=\left(\begin{array}{l}
0 \\
1
\end{array}\right)-\int_{-\infty}^{x}\left(\begin{array}{cc}
0 & -e^{-2 i k\left(x-x^{\prime}\right)} \\
1 & 0
\end{array}\right)\left(\begin{array}{ll}
p & 0 \\
0 & q
\end{array}\right) f\left(x^{\prime}, k\right) d x^{\prime}
$$

и, поскольку интегральное ядро экспоненциально убывает при $\operatorname{Im} k<0$, ряд Неймана для решения уравнения сходится в этой полуплоскости.

Отсюда следует, что

$T(k)$ аналитически продолжается на верхнюю полуплоскость $\operatorname{Im} k \geqslant 0$.

Доказывается, что

a) $a(k)$ нигде не равно нулю на $\mathbb{R} \backslash\{0\}$;

б) полюсы функции $T(k)$ отвечают связанным состояниям, т.е. решениям уравнения (68), которые убывают экспоненциально при $x \rightarrow \pm \infty$; эти решения суть $\varphi_{1}^{+}(x, \varkappa)$ и $\varphi_{2}^{-}(x, \varkappa)$, где $a(\varkappa)=0$ и, следовательно,

$$
\varphi_{2}^{-}(x, \varkappa)=\mu(\varkappa) \varphi_{1}^{+}(x, \varkappa), \quad \mu(\varkappa) \in \mathbb{C},
$$

и кратность каждого собственного значения $\varkappa$ равна единице;

в) $T(k)$ имеет только простые полюсы в полуплоскости $\operatorname{Im} k>0$ и для экспоненциально убывающих потенциалов число таких полюсов конечно; 
г) поскольку множество решений уравнения (68) инвариантно относительно (71), дискретный спектр оператора $L$ сохраняется комплексным сопряжением $\varkappa \rightarrow \bar{\varkappa}$ и образован полюсами функции $T(k)$ и их комплексными сопряжениями.

Спектральные данные оператора $L$ состоят из

1) коэффициента отражения $R(k), k \neq 0$;

2) полюсов функции $T(k)$ в полуплоскости $\operatorname{Im} \varkappa>0: \varkappa_{1}, \ldots, \varkappa_{N}$;

3) величин $\lambda_{j}=i \gamma_{j} \mu_{j}, j=1, \ldots, N$, где $\gamma_{j}=\gamma\left(\varkappa_{j}\right)$ - вычет $T(k)$ в $\varkappa_{j}$ и $\mu_{j}=\mu\left(\varkappa_{j}\right)($ см. $(73))$.

Если потенциал $p=\bar{q}$ вещественнозначный, то

$$
\varphi_{j}^{ \pm}(x,-k)=\overline{\varphi_{j}^{ \pm}(x, k)} \quad \text { для } \quad k \in \mathbb{R} \backslash\{0\},
$$

и это влечет, что

$$
a(k)=\overline{a(-k)}, \quad R(k)=\overline{R(-k)}, \quad T(k)=\overline{T(-\bar{k})},
$$

полюсы $T(k)$ симметричны относительно мнимой оси и

$$
\lambda_{j}=\bar{\lambda}_{k} \quad \text { для } \quad \varkappa_{j}=-\bar{\varkappa}_{k} .
$$

Теперь применяя преобразование Фурье (относительно $k$ ) к обеим частям равенства

$$
T(k) \varphi_{2}^{-}=R(k) \varphi_{1}^{+}+\varphi_{2}^{+},
$$

после некоторых подстановок мы запишем (74) для компонент вектор-функций в виде уравнений Гельфанда-Левитана-Марченко

$$
\begin{gathered}
B_{2}(x, y)+\int_{x}^{+\infty} B_{1}\left(x, x^{\prime}\right) \Omega\left(x^{\prime}+y\right) d x^{\prime}=0, \\
\Omega(x+y)-B_{1}(x, y)+\int_{x}^{+\infty} B_{2}\left(x, x^{\prime}\right) \Omega\left(x^{\prime}+y\right) d x^{\prime}=0
\end{gathered}
$$

на $B_{1}$ и $B_{2}$, где

$$
\Omega(z)=\frac{1}{2 \pi} \int_{-\infty}^{+\infty} R(k) e^{-i k z} d k-\sum_{j=1}^{N} \lambda_{j} e^{i \varkappa_{j} z}
$$

при $y>x$ и существуют следующие пределы:

$$
\lim _{y \rightarrow \infty} B_{k}(x, y)=0, \quad \lim _{y \rightarrow x+} B_{k}(x, y)=B_{k}(x, x), \quad k=1,2 .
$$

Это - уравнения вольтерровского типа, и они однозначно разрешимы. Формулы восстановления для потенциалов имеют вид

$$
p(x)=-2 B_{1}(x, x), \quad p(x) q(x)=p(x) \overline{p(x)}=2 \frac{d B_{2}(x, x)}{d x} .
$$

См. подробный вывод этих формул из [135], например, в [138].

В дальнейшем, для простоты, мы предположим, что потенциал $p(x)$ принимает только вещественные значения. 
В [120] выведена серия формул, выражающая интегралы Крускала через спектральные данные, так называемые формулы следов. Мы упомянем только формулу для первого нетривиального интеграла:

$$
\int_{-\infty}^{\infty} p^{2}(x) d x=-\frac{1}{\pi} \int_{-\infty}^{\infty} \log \left(1-|b(k)|^{2}\right) d k+4 \sum_{j=1}^{N} \operatorname{Im} \varkappa_{j}
$$

Для безотражательных потенциалов процедура восстановления сводится к алгебраическим уравнениям (см. подробности, например, в [135], [138], [6]). Спектральные данные состоят из полюсов $\varkappa_{k}$ и соответствующих величин $\lambda_{j}$, $j=1, \ldots, N$. Пусть

$$
\begin{gathered}
\Psi(x)=\left(-\lambda_{1} e^{i \varkappa_{1} x}, \ldots,-\lambda_{N} e^{i \varkappa_{N} x}\right), \\
M_{j k}(x)=\frac{\lambda_{k}}{i\left(\varkappa_{j}+\varkappa_{k}\right)} e^{i\left(\varkappa_{j}+\varkappa_{k}\right) x}, \quad j, k=1, \ldots, N .
\end{gathered}
$$

Мы имеем

$$
\begin{aligned}
p(x) & =2 \frac{d}{d x} \arctan \frac{\operatorname{Im} \operatorname{det}(1+i M(x))}{\operatorname{Redet}(1+i M(x))}, \\
\varphi_{1}^{+}(x, k) & =\left(\begin{array}{c}
\left\langle\Psi(x) \cdot\left(1+M^{2}(x)\right)^{-1} \mid W(x, k)\right\rangle \\
e^{i k x}-\left\langle\Psi(x) \cdot\left(1+M^{2}(x)\right)^{-1} M(x) \mid W(x, k)\right\rangle
\end{array}\right),
\end{aligned}
$$

где $\langle u \mid v\rangle=u_{1} v_{1}+\cdots+u_{N} v_{N}$ и

$$
W(x, k)=\left(\frac{i}{\varkappa_{1}+k} e^{i\left(\varkappa_{1}+k\right) x}, \ldots, \frac{i}{\varkappa_{N}+k} e^{i\left(\varkappa_{N}+k\right) x}\right) .
$$

ЗАКЛЮчИТЕЛЬНОЕ ЗАмЕчАНИЕ. Эта работа была начата и значительная часть ее была написана во время пребывания автора в Математическом институте Макса Планка в октябре 2003 - январе 2004 г., а окончательная корректура была проведена в этом же институте в декабре 2005 г.

\section{Список литературы}

[1] I. A. Taimanov, "Modified Novikov-Veselov equation and differential geometry of surfaces", Amer. Math. Soc. Transl. Ser. 2, 179 (1997), 133-151; http://arxiv.org/dg-ga/9511005.

[2] B. G. Konopelchenko, "Induced surfaces and their integrable dynamics", Stud. Appl. Math., 96:1 (1996), 9-51.

[3] И.А. Тайманов, "Представление Вейерштрасса замкнутых поверхностей в $\mathbb{R}^{3 "}$, Функи. анализ и его прил., 32:4 (1998), 49-62.

[4] Б. А. Дубровин, И. М. Кричевер, С. П. Новиков, "Уравнение Шрёдингера в периодическом поле и римановы поверхности”, Докл. АН CCCP, 229:1 (1976), 15-19.

[5] M. U. Schmidt, A proof of the Willmore conjecture, SFB 256, Preprint no. 546, Technischen Universität Berlin, 2002; http://arxiv.org/math.DG/0203224.

[6] И.А. Тайманов, "Представление Вейерштрасса сфер в $\mathbb{R}^{3}$, числа Уиллмора и солитонные сферы", Труды МИАН, 225 (1999), 339-361.

[7] D. Ferus, K. Leschke, F. Pedit, U. Pinkall, "Quaternionic holomorphic geometry: Plücker formula, Dirac eigenvalue estimates and energy estimates of harmonic 2tori", Invent. Math., 146:3 (2001), 507-593. 
[8] F. Pedit, U. Pinkall, "Quaternionic analysis on Riemann surfaces and differential geometry", Doc. Math., J. DMV Extra Vol. ICM, II (1998), 389-400.

[9] B. G. Konopelchenko, "Weierstrass representations for surfaces in 4D spaces and their integrable deformations via DS hierarchy", Ann. Global Anal. Geom., 16:1 (2000), 61-74.

[10] И. А. Тайманов, "Операторы Дирака и конформные инварианты торов в трехмерном пространстве", Труды МИАН, 244 (2004), 249-280.

[11] Д. А. Бердинский, И. А. Тайманов, "Поверхности в трехмерных группах Ли", Сиб. матем. журн., 46:6 (2005), 1248-1264.

[12] F. E. Burstall, D. Ferus, K. Leschke, F. Pedit, U. Pinkall, Conformal geometry of surfaces in $S^{4}$ and quaternions, Lecture Notes in Math., 1772, Springer-Verlag, Berlin, 2002.

[13] W. Helfrich, "Elastic properties of lipid bilayers: theory and possible experiments", Z. Naturforsch. C, 28 (1973), 693-703.

[14] E. M. Blokhius, W.F.C. Sager, "Helfrich energy for aggregation and adhesion", J. Chemical Phys., 110 (1999), 3148-3152.

[15] C.-H. Lin, M.-H. Lo, Y.-C. Tsai, "Shape energy of fluid membranes", Progr. Theoret. Phys., 109:4 (2003), 591-618.

[16] L.P. Eisenhart, A Treatise on the differential geometry of curves and surfaces, Ginn, Boston, 1909.

[17] T. Friedrich, "On the spinor representation of surfaces in Euclidean 3-space", J. Geom. Phys., 28:1-2 (1998), 143-157.

[18] S. Matsutani, "Immersion anomaly of Dirac operator on surface in $\mathbb{R}^{3 "}$, Rev. Math. Phys., 11:2 (1999), 171-186.

[19] K. Kenmotsu, "Weierstrass formula for surfaces of prescribed mean curvature", Math. Ann., 245:2 (1979), 89-99.

[20] P. Scott, "The geometries of 3-manifolds", Bull. London Math. Soc., 15:5 (1983), 401-487.

[21] W.P. Thurston, "Three-dimensional manifolds, Kleinian groups and hyperbolic geometry", Bull. Amer. Math. Soc. (N.S.), 6:3 (1982), 357-379.

[22] U. Abresch, "Generalized Hopf differentials", Meeting on Geometry (Oberwolfach, 2004).

[23] U. Abresch, H. Rosenberg, "A Hopf differential for constant mean curvature surfaces in $S^{2} \times R$ and $H^{2} \times R$ ", Acta Math., 193:2 (2004), 141-174.

[24] I. Fernandez, P. Mira, "A characterization of constant mean curvature surfaces in homogeneous 3-manifolds", http://arxiv.org/math.DG/0512280.

[25] J.-I. Inoguchi, T. Kumamoto, N. Ohsugi, Y. Suyama, "Differential geometry of curves and surfaces in 3-dimensional homogeneous spaces. II", Fukuoka Univ. Sci. Rep., 30:1 (2000), 17-47.

[26] J. Inoguchi, "Minimal surfaces in 3-dimensional solvable Lie groups", Chinese Ann. Math. Ser. B, 24:1 (2003), 73-84.

[27] B. Daniel, "Isometric immersions into 3-dimensional homogeneous manifolds", http://arxiv.org/math.DG/0503500.

[28] A. S. Fokas, I. M. Gelfand, "Surfaces on Lie groups, on Lie algebras, and their integrability", Comm. Math. Phys., 177:1 (1996), 203-220.

[29] G. Kamberov, P. Norman, F. Pedit, U. Pinkall, Quaternions, spinors, and surfaces, Contemp. Math., 299, Amer. Math. Soc., Providence, RI, 2002.

[30] G. Kamberov, F. Pedit, U. Pinkall, "Bonnet pairs and isothermic surfaces", Duke Math. J., 92:3 (1998), 637-644.

[31] J. Richter, Conformal maps of a Riemann surface into the space of quaternions, Ph.D. Thesis, Technische Universität Berlin, 1997. 
[32] K. Leschke, F. Pedit, U. Pinkall, "Willmore tori in the 4-sphere with nontrivial normal bundle", Math. Ann., 332:2 (2005), 381-394.

[33] F. Hélein, P. Romon, "Weierstrass representation of Lagrangian surfaces in fourdimensional space using spinors and quaternions", Comment. Math. Helv., 75:4 (2000), 668-680.

[34] F. Hélein, "On Konopelchenko's representation for surfaces in 4 dimensions", http://arxiv.org/math.DG/0104101.

[35] I. A. Taimanov, "Surfaces in the four-space and the Davey-Stewartson equations", J. Geom. Phys., 56:8 (2006), 1235-1256.

[36] Л.В. Богданов, "Уравнение Веселова-Новикова как естественное двумерное обобщение уравнения Кортевега-де Фриза”, ТМФ, 70:2 (1987), 309-314.

[37] Л.В.Богданов, “О двумерной задаче Захарова-Шабата”, ТМФ, 72:1 (1987), 155159.

[38] А. П. Веселов, С.П. Новиков, "Конечнозонные двумерные потенциальные операторы Шрёдингера. Явные формулы и эволюционные уравнения", Докл. АН CCCP, 279:1 (1984), 20-24.

[39] А. П. Веселов, С. П. Новиков, "Конечнозонные двумерные операторы Шрёдингера. Потенциальные операторы", Докл. АН СССР, 279:4 (1984), 784-788.

[40] И. А. Тайманов, "Конечнозонные решения модифицированных уравнений Веселова-Новикова, их спектральные свойства и приложения", Сиб. матем. журн., 40:6 (1999), 1352-1363.

[41] И. А. Тайманов, “О двумерных конечнозонных потенциальных операторах Шрёдингера и Дирака с особыми спектральными кривыми”, Сиб. матем. журн., 44:4 (2003), 870-882.

[42] F. Burstall, F. Pedit, U. Pinkall, "Schwarzian derivatives and flows of surfaces", Differential Geometry and Integrable Systems (Tokyo, 2000), Contemp. Math., 308, Amer. Math. Soc., Providence, RI, 2002, 39-61.

[43] B. G. Konopelchenko, U. Pinkall, "Integrable deformations of affine surfaces via the Nizhik-Veselov-Novikov equation", Phys. Lett. A, 245:3-4 (1998), 239-245.

[44] E. V. Ferapontov, "Surfaces with flat normal bundle: an explicit construction", Differential Geom. Appl., 14:1 (2001), 15-37.

[45] L. V. Bogdanov, E. V. Ferapontov, "Projective differential geometry of higher reductions of the two-dimensional Dirac equation", J. Geom. Phys., 52:3 (2004), 328-352.

[46] I. A. Taimanov, "Surfaces of revolution in terms of solitons", Ann. Global Anal. Geom., 12:5 (1997), 419-435.

[47] J. Langer, "Recursion in curve geometry", New York J. Math., 5 (1999), 25-51.

[48] O. Garay, J. Langer, "Taimanov's surface evolution and Bäcklund transformations for curves", Conform. Geom. Dyn., 3 (1999), 37-49.

[49] М. В. Келдыш, "О собственных значениях и собственных функциях некоторых классов несамосопряженных уравнений”, Докл. АН СССР, 77:1 (1951), 11-14.

[50] М. В. Келдыш, "О полноте собственных функций некоторых классов несамосопряженных линейных операторов", УМН, 26:4 (1971), 15-41.

[51] H. Grauert, R. Remmert, Coherent Analytic Sheaves, Grundlehren Math. Wiss., 265, Springer-Verlag, Berlin, 1984.

[52] И. М. Кричевер, "Спектральная теория двумерных периодических операторов и ее приложения", УМН, 44:2 (1989), 121-184.

[53] П. А. Кучмент, “Теория Флоке для уравнений в частных производных", УМН, 37:4 (1982), 3-52.

[54] P. Kuchment, Floquet theory for partial differential equations, Oper. Theory Adv. Appl., 60, Birkhäuser, Basel, 1993. 
[55] I. A. Taimanov, "Finite-gap theory of the Clifford torus", Internat. Math. Res. Notices, 2 (2005), 103-120.

[56] С. П. Новиков, "Периодическая задача Кортевега-де Фриза", Функи. анализ и его прил., 8:3 (1974), 54-66.

[57] С. В. Манаков, "Метод обратной задачи и двумерные солитонные уравнения", УМH, 31:5 (1976), 245-246.

[58] J. Feldman, H. Knörrer, E. Trubowitz, "There is no two-dimensional analogue of Lamé's equation", Math. Ann., 294:2 (1992), 295-324.

[59] J. Feldman, H. Knörrer, E. Trubowitz, Riemann surfaces of infinite genus, CRM Monogr. Ser., 20, Amer. Math. Soc., Providence, RI, 2003.

[60] И. М. Кричевер, "Методы алгебраической геометрии в теории нелинейных уравнений", УМН, 32:6 (1977), 183-208.

[61] P. G. Grinevich, M. U. Schmidt, "Conformal invariant functionals of immersions of tori into $\mathbb{R}^{3 ",}$ J. Geom. Phys., 26:1-2 (1998), 51-78.

[62] N. J. Hitchin, "Harmonic maps from a 2-torus to the 3-sphere", J. Differential Geom., 31:3 (1990), 627-710.

[63] K. Pohlmeyer, "Integrable Hamiltonian systems and interactions through constraints", Comm. Math. Phys., 46:3 (1976), 207-221.

[64] В. Е. Захаров, А. В. Михайлов, "Релятивистски-инвариантные двумерные модели теории поля, интегрируемые методом обратной задачи рассеяния", ЖЭТФ, 74:6 (1978), 1953-1973.

[65] K. Uhlenbeck, "Harmonic maps into Lie groups: classical solutions of the chiral model", J. Differential Geom., 30:1 (1989), 1-50.

[66] M. A. Guest, Harmonic maps, loop groups, and integrable systems, London Math. Soc. Stud. Texts, 38, Cambridge Univ. Press, Cambridge, 1997.

[67] A.P. Fordy, J.C. Wood (eds.), Harmonic maps and integrable systems, Aspects Math., E23, Vieweg, Braunschweig, 1994.

[68] F. Hélein, Constant mean curvature surfaces, harmonic maps and integrable systems. Notes taken by Roger Moser, Lectures Math. ETH Zurich, Birkhäuser, Basel, 2001.

[69] A. I. Bobenko, "Surface in terms of 2 by 2 matrices. Old and new integrable cases", Harmonic Maps and Integrable Systems, Aspects Math., E23, eds. A.P. Fordy, J. C. Wood, Vieweg, Braunschweig, 1994, 83-127.

[70] A. S. Fokas, I. M. Gel'fand, F. Finkel, Q. M. Liu, "A formula for constructing infinitely many surfaces on Lie algebras and integrable equations", Selecta Math. (N.S.), 6:4 (2000), 347-375.

[71] E. A. Ruh, J. Vilms, "The tension field of the Gauss map", Trans. Amer. Math. Soc., 149 (1970), 569-573.

[72] H. Hopf, Differential geometry in the large, Lecture Notes in Math., 1000, SpringerVerlag, Berlin, 1983.

[73] A. D. Alexandrov, "A characteristic property of spheres", Ann. Mat. Pura Appl. (4), 58 (1962), 303-315.

[74] H. C. Wente, "Counterexample to a conjecture of H. Hopf", Pacific J. Math., 121:1 (1986), 193-243.

[75] U. Abresch, "Constant mean curvature tori in terms of elliptic functions", J. Reine Angew. Math., 374 (1987), 169-192.

[76] U. Abresch, "Old and new doubly periodic solutions of the sinh-Gordon equation", Seminar on new results in nonlinear partial differential equations (Bonn, 1984), Aspects Math., E10, Vieweg, Braunschweig, 1987, 37-73.

[77] U. Pinkall, I. Sterling, "On the classification of constant mean curvature tori", Ann. of Math. (2), 130:2 (1989), 407-451. 
[78] A. I. Bobenko, "All constant mean curvature tori in $\mathbb{R}^{3}, S^{3}, H^{3}$ in terms of thetafunctions", Math. Ann., 290:2 (1991), 209-245.

[79] А.И. Бобенко, "Поверхности постоянной средней кривизны и интегрируемые уравнения", УМН, 46:4 (1991), 3-42.

[80] N. Kapouleas, "Complete constant mean curvature surfaces in Euclidean threespace", Ann. of Math. (2), 131:2 (1990), 239-330.

[81] N. Kapouleas, "Constant mean curvature surfaces constructed by fusing Wente tori", Invent. Math., 119:3 (1995), 443-518.

[82] B. G. Konopelchenko, I. A. Taimanov, "Constant mean curvature surfaces via an integrable dynamical system", J. Phys. A, 29:6 (1996), 1261-1265.

[83] F. Ehlers, H. Knörrer, "An algebro-geometric interpretation of the Bäcklund transformation for the Korteweg-de Vries equation", Comment. Math. Helv., 57:1 (1982), $1-10$.

[84] T. J. Willmore, "Note on embedded surfaces", An. Ştiinţ. Univ. Al. I. Cuza Iaşi Secţ. I a Mat. (N.S.), 11B (1965), 493-496.

[85] G. Thomsen, "Über konforme Geometrie. I: Grundlangen der konformen Flächentheorie", Abh. Math. Sem. Univ. Hamburg, 3 (1923), 31-56.

[86] W. Blaschke, Vorlesungen über Differentialgeometrie und geometrische Grundlagen von Einsteins Relativitätstheorie, III: Differentialgeometrie der Kreise und Kugeln, Springer-Verlag, Berlin, 1929.

[87] R. L. Bryant, "A duality theorem for Willmore surfaces", J. Differential Geom., 20:1 (1984), 23-53.

[88] U. Pinkall, "Hopf tori in $S^{3 ", ~ I n v e n t . ~ M a t h ., ~ 81: 2 ~(1985), ~ 379-386 . ~}$

[89] M. Babich, A. Bobenko, "Willmore tori with umbilic lines and minimal surfaces in hyperbolic space", Duke Math. J., 72:1 (1993), 151-185.

[90] L. Simon, "Existence of surfaces minimizing the Willmore functional", Comm. Anal. Geom., 1:2 (1993), 281-326.

[91] M. Bauer, E. Kuwert, "Existence of minimizing Willmore surfaces of prescribed genus", Internat. Math. Res. Notices, 10 (2003), 553-576.

[92] M. U. Schmidt, Existence of minimizing Willmore surfaces of prescribed conformal class, http://arxiv.org/math.DG/0403301.

[93] C.-K. Peng, L. Xiao, "Willmore surfaces and minimal surfaces with flat ends", Geometry and Topology of Submanifolds, X (Beijing/Berlin, 1999), World Scientific, River Edge, NJ, 2000, 259-265.

[94] R. L. Bryant, "Surfaces in conformal geometry", The Mathematical heritage of Hermann Weyl (Durham, 1987), Proc. Sympos. Pure Math., 48, Amer. Math. Soc., Providence, RI, 1988, 227-240.

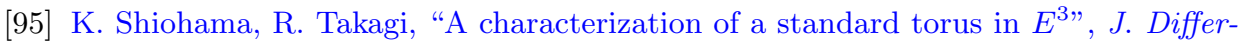
ential Geom., 4 (1970), 477-485.

[96] T. J. Willmore, "Mean curvature of Riemannian immersions", J. London Math. Soc. (2), 3 (1971), 307-310.

[97] U. Hertrich-Jeromin, U. Pinkall, "Ein Beweis des Willmoreschen Vermutung für Kanaltori", J. Reine Angew. Math., 430 (1992), 21-34.

[98] J. Langer, D. Singer, "Curves in the hyperbolic plane and mean curvature of tori in 3-space", Bull. London Math. Soc., 16:5 (1984), 531-534.

[99] P. Li, S. T. Yau, "A new conformal invariant and its applications to the Willmore conjecture and the first eigenvalue for compact surfaces", Invent. Math., 69:2 (1982), 269-291.

[100] S. Montiel, A. Ros, "Minimal immersions of surfaces by the first eigenfunctions and conformal area", Invent. Math., 83:1 (1986), 153-166. 
[101] A. Ros, "The Willmore conjecture in the real projective space", Math. Res. Lett., 6:5-6 (1999), 487-493.

[102] B. Ammann, "The Willmore conjecture for immersed tori with small curvature integral", Manuscripta Math., 101:1 (2000), 1-22.

[103] P. Topping, "Towards the Willmore conjecture", Calc. Var. Partial Differential Equations, 11:4 (2000), 361-393.

[104] J. L. Weiner, "On a problem of Chen, Willmore, et al.", Indiana Univ. Math. J., 27:1 (1978), 19-35.

[105] B. Palmer, "The conformal Gauss map and the stability of Willmore surfaces", Ann. Global Anal. Geom., 9:3 (1991), 305-317.

[106] R. Kusner, N. Schmitt, "The spinor representation of minimal surfaces", GANG Preprint 3.27, Center for Geometry, Analysis, Numerics \& Graphics, Amherst, MA, 1994; http://www.gans. umass.edu.

[107] C. J. Costa, "Complete minimal surfaces in $\mathbb{R}^{3}$ of genus one and four planar embedded ends", Proc. Amer. Math. Soc., 119:4 (1993), 1279-1287.

[108] Э. И. Шамаев, "Об одном семействе минимальных торов с плоскими концами в $\mathbb{R}^{3 ",, ~ С и б . ~ м а т е м . ~ ж у р н ., ~ 46: 6 ~(2005), ~ 1407-1426 . ~}$

[109] D. Ferus, F. Pedit, U. Pinkall, I. Sterling, "Minimal tori in $S^{4}$ ", J. Reine Angew. Math., 429 (1992), 1-47.

[110] M. V. Babich, "Willmore surfaces, 4-particle Toda lattice and double coverings of hyperelliptic surfaces", Amer. Math. Soc. Transl. Ser. 2, 174 (1996), 143-168.

[111] F. Hélein, "Willmore immersions and loop groups", J. Differential Geom., 50:2 (1998), 331-385.

[112] R. Kusner, "Comparison surfaces for the Willmore problem", Pacific J. Math., 138:2 (1989), 317-345.

[113] P. Wintgen, "On the total curvature of surfaces in $E^{4 ",}$ Colloq. Math., 39:2 (1978), 289-296.

[114] P. Wintgen, "Sur l'inégalité de Chen-Willmore", C. R. Acad. Sci. Paris Ser. A, 288:21 (1979), 993-995.

[115] W. P. Minicozzi II, "The Willmore functional of Lagrangian tori: its relation to area and existence of smooth minimizers", J. Amer. Math. Soc., 8:4 (1995), 761-791.

[116] B.-Y. Chen, "Some conformal invariants of submanifolds and their applications", Boll. Unione. Mat. Ital. (4), 10 (1974), 380-385.

[117] E. Kuwert, R. Schatzle, "Removability of point singularities of Willmore surfaces", Ann. of Math. (2), 160:1 (2004), 315-357.

[118] U. Pinkall, I. Sterling, "Willmore surfaces", Math. Intelligencer, 9:2 (1987), 38-43.

[119] C. Bohle, G.P. Peters, U. Pinkall, "Constrained Willmore surfaces", http://arxiv.org/math.DG/0411479.

[120] Л. А. Тахтаджян, Л. Д. Фаддеев, Гамильтонов подход в теории солитонов, Наука, М., 1986.

[121] T. Friedrich, "Eigenvalues of the Dirac operator on Riemannian manifolds", Conference on Differential Geometry (Brno, August 1998).

[122] C. Bär, "Lower eigenvalue estimates for Dirac operators", Math. Ann., 293:1 (1992), 39-46.

[123] T. Friedrich, Dirac operators in Riemannian geometry, Graduate Studies in Math., 25, Amer. Math. Soc., Providence, RI, 2000.

[124] H.-B. Lawson Jr., M.-L. Michelsohn, Spin Geometry, Princeton Univ. Press, Princeton, NJ, 1989.

[125] H. Baum, T. Friedrich, R. Grunewald, I. Kath, Twistors and Killing spinors on Riemannian manifolds, Teubner-Texte Math., 124, Teubner, Stuttgart, 1991. 
[126] N. Aronszajn, "A unique continuation theorem for solutions of elliptic partial differential equations or inequalities of second order", J. Math. Pures Appl. (9), 36 (1957), 235-249.

[127] P. Hartman, A. Wintner, "On the local behavior of solutions of non-parabolic differential equations", Amer. J. Math., 75 (1953), 449-476.

[128] Ф. Гриффитс, Дж. Харрис, Приниипы алгебраической геометрии, 1, 2, Мир, M., 1982.

[129] M. Haskins, "The geometric complexity of special Lagrangian $T^{2}$-cones", Invent. Math., 157:1 (2004), 11-70.

[130] G.P. Peters, Soliton spheres, Ph.D. Thesis, Technische Universität Berlin, 2004; http://edocs.tu-berlin.de/diss/.

[131] C. Bohle, G.P. Peters, "Bryant surfaces with smooth ends", http://arxiv.org/math.DG/0411480.

[132] R. L. Bryant, "Surfaces of mean curvature one in hyperbolic space", Théorie des variétés minimales et applications (Palaiseau, 1983-1984), Astérisque, 154-155, 1987, 321-347.

[133] J.-H. Eschenburg, "Willmore surfaces and Moebius geometry", Preprint, Augsburg University, 1988.

[134] U. Hertrich-Jeromin, Introduction to Möbius differential geometry, London Math. Soc. Lecture Note Ser., 300, Cambridge Univ. Press, Cambridge, 2003.

[135] В. Е. Захаров, А. Б. Шабат, "Точная теория двумерной самофокусировки и одномерной автомодуляции волн в нелинейных средах", ЖЖЭТФ, 61:1 (1971), 118-134.

[136] Л.Д. Фаддеев, "Свойства $S$-матрицы одномерного оператора Шрёдингера", Труды МИАН, 73 (1964), 314-336.

[137] В.А. Марченко, Операторы Штурма-Лиувилля и их приложения, Наукова Думка, Киев, 1977.

[138] М. Абловиц, Х. Сигур, Солитонъ и метод обратной задачи, Мир, М., 1987.

И. А. Тайманов (I. А. Taimanov)

Институт математики, Новосибирск

E-mail: taimanov@math.nsc.ru
Поступила в редакцию

30.11 .2005 